\title{
TUNNELING AND METASTABILITY OF CONTINUOUS TIME MARKOV CHAINS
}

\author{
J. BELTRÁN, C. LANDIM
}

\begin{abstract}
We propose a new definition of metastability of Markov processes on countable state spaces. We obtain sufficient conditions for a sequence of processes to be metastable. In the reversible case these conditions are expressed in terms of the capacity and of the stationary measure of the metastable states.
\end{abstract}

\section{INTRODUCTION}

In the framework of non-equilibrium statistical mechanics, metastability is a relevant dynamical phenomenon taking place in the vicinities of first order phase transitions. There has been along the years several proposals of a rigorous mathematical description of the phenomenon starting with Lebowitz and Penrose [16] who derived the canonical free energy for Kac potentials in the Van der Waals limit. The seminal paper of Cassandro, Galves, Olivieri and Vares [6] proposed a pathwise approach to metastability which highlighted the underlying Markov structure behind metastability which is exploited here. In the sequel, Scoppola 19] examined the metastable behavior of finite state space Markov chains with transition probabilities exponentially small in a parameter. More recently, Bovier and co-authors (5] and references therein) presented a new approach based on the spectral properties of the generator of the process. We refer to [18 for a recent monograph on the subject.

We propose in this article an alternative formulation of metastability for sequences of Markov processes on countable state spaces. Informally, a process is said to exhibit a metastable behavior if it remains for a very long time in a state before undergoing a rapid transition to a stable state. After the transition, the process remains in the stable state for a period of time much longer than the time spent in the first state, called for this reason metastable. In certain cases, there are two or more "metastable wells" with the same depth, a situation called by physicists "competing metastable states". In these cases, the process thermalizes in each well before jumping abruptly to another well where the same qualitative behavior is observed.

To describe our approach, denote by $E_{N}, N \geq 1$, a sequence of countable spaces and by $\left(\theta_{N}: N \geq 1\right)$ a sequence of positive real numbers. For each $N \geq 1$, consider a partition $\varepsilon_{N}^{1}, \ldots, \varepsilon_{N}^{\kappa}, \Delta_{N}$ of $E_{N}$ and a $E_{N}$-valued Markov process $\left\{\eta_{t}^{N}: t \geq 0\right\}$. Fix a state $\xi_{x}^{N}$ in $\mathcal{E}_{N}^{x}, 1 \leq x \leq \kappa$. We say that the sequence of Markov processes $\left\{\eta_{t}^{N}: t \geq 0\right\}, N \geq 1$, exhibits a tunneling behavior in the time scale $\left(\theta_{N}: N \geq\right.$ 1) with metastates $\mathcal{E}_{N}^{1}, \ldots, \mathcal{E}_{N}^{\kappa}$, attractors $\xi_{1}^{N}, \ldots, \xi_{\kappa}^{N}$, and asymptotic behavior

Key words and phrases. Meta-stability, Markov processes, condensation, zero-range processes. 
described by the Markov process on $S=\{1, \ldots, \kappa\}$ with rates $\{r(x, y): x, y \in S\}$ if the following three conditions are fulfilled:

(1) For every $1 \leq x \leq \kappa$, starting from a state $\eta^{N}$ in $\mathcal{E}_{N}^{x}$, with overwhelming probability, the process $\left\{\eta_{t}^{N}: t \geq 0\right\}$ reaches $\xi_{x}^{N}$ before attaining $\bigcup_{y \neq x} \varepsilon_{N}^{y}$.

(2) Let $\left\{X_{t}^{N}: t \geq 0\right\}$ be the process $X_{t}^{N}=\Psi_{N}\left(\eta_{t}^{\mathcal{E}_{N}}\right)$, where $\left\{\eta_{t}^{\mathcal{E}_{N}}: t \geq 0\right\}$ is the trace of the Markov process $\left\{\eta_{t}: t \geq 0\right\}$ on $\mathcal{E}_{N}=\bigcup_{1<x<\kappa} \mathcal{E}_{N}^{x}$ and where $\Psi_{N}(\eta)=\sum_{1 \leq x \leq \kappa} x \mathbf{1}\left\{\eta \in \mathcal{E}_{N}^{x}\right\}$. The speed up process $\left\{X_{t \theta_{N}}^{N}: t \geq 0\right\}$ converges to the Markov process on $S$ which jumps from $x$ to $y$ at rate $r(x, y)$.

(3) Starting from any point of $\mathcal{E}_{N}$, the time spent by the speed up Markov process $\left\{\eta_{t \theta_{N}}: t \geq 0\right\}$ on the set $\Delta_{N}$ in any time interval $[0, s], s>0$, vanishes in probability.

All the terminology used in the previous definition is explained in the next section. Condition (1) states that the process thermalizes in each set $\mathcal{E}_{N}^{x}$ before reaching another metastate set $\mathcal{E}_{N}^{y}, y \neq x$. The assumption of the existence of an attractor can clearly be relaxed, but is satisfied in several interesting examples, as in the condensed zero-range processes 3 , 4, which motivated the present work. Condition (2) describes the intervalley dynamics and reveals the loss of memory of the jump times from a well to another, put in evidence in [6]. In condition (3) we assume that the starting point belongs to $\mathcal{E}_{N}$. It may therefore happen that the discarded set $\Delta_{N}$ hides wells deeper than the wells $\mathcal{E}_{N}^{x}, 1 \leq x \leq \kappa$, but which can not be attained from $\mathcal{E}_{N}$. When we remove in this condition the assumption that the starting point belongs to $\mathcal{E}_{N}$, we say that the process exhibits a metastable behavior, instead of a tunneling behavior. In this case, the wells $\mathcal{E}_{N}^{x}, 1 \leq x \leq \kappa$ are the deepest ones.

In contrast with the pathwise approach to metastability [6], the present one does not give a precise description of the saddle points between the wells nor of the typical path which drives the system from one well to another. Its description of metastability is in some sense rougher, but keeps the main ingredients, as thermalization and asymptotic Markovianity.

The main results of this article, stated in the next section, establish sufficient conditions for recurrent Markov processes on countable state spaces to exhibit a tunneling behavior. In the reversible case, these sufficient conditions can be expressed in terms of the capacity and of the stationary probability measure of the metastates.

A theory is meaningless if no interesting example is provided which fits in the framework presented. Besides the mean field models considered in [6] and the Freidlin-Wentsell Markov chains proposed in [18, which naturally enter in the present framework, we examine in [3, 4] a new class of processes which exhibit a metastable behavior. This family, known as the condensed zero-range processes, have been introduced in the physics literature [8, 13, 9] to model the Bose-Einstein condensation phenomena. It has been proved in several different contexts [14, 10, 1] that, above a critical density, all but a small number of particles concentrate on one single site in the canonical stationary states of these processes. In [3, 4] we prove that, in the reversible case, the condensed zero range processes exhibit a tunneling behavior by showing that in an appropriate time scale the condensed site evolves according to a random walk on $S$. We also prove that the jump rates of 
the asymptotic Markov dynamics can be expressed in terms of the capacities of the underlying random walks performed by the particles.

The article is organized as follows. In Section 2] we introduce the notation, the definitions and state the main theorems. In Section 3 we present some elementary examples which justify the definitions proposed. In Sections 4, 5, we prove the main results. Finally, in Section 6, we prove some results on the trace of Markov processes needed in the article and which we did not find in the literature.

\section{Notation and Results}

Fix a sequence $\left(E_{N}: N \geq 1\right)$ of countable state spaces. The elements of $E_{N}$ are denoted by the Greek letters $\eta$, $\xi$. For each $N \geq 1$ consider a matrix $R_{N}$ : $E_{N} \times E_{N} \rightarrow \mathbb{R}$ such that $R_{N}(\eta, \xi) \geq 0$ for $\eta \neq \xi,-\infty<R_{N}(\eta, \eta) \leq 0$ and $\sum_{\xi \in E_{N}} R_{N}(\eta, \xi)=0$ for all $\eta \in E_{N}$. Denote by $L_{N}$ the generator which acts on bounded functions $f: E_{N} \rightarrow \mathbb{R}$ as

$$
\left(L_{N} f\right)(\eta)=\sum_{\xi \in E_{N}} R_{N}(\eta, \xi)\{f(\xi)-f(\eta)\} .
$$

Let $\left\{\eta_{t}^{N}: t \geq 0\right\}$ be the minimal right-continuous Markov process associated to the generator $L_{N}$. We refer to [7, 11, 17] for the terminology and the main facts on Markov processes alluded to in this article. It is well known, for instance, that $\left\{\eta_{t}^{N}: t \geq 0\right\}$ is a strong Markov process with respect to the filtration $\left\{\mathcal{F}_{t}^{N}: t \geq 0\right\}$ given by $\mathcal{F}_{t}^{N}=\sigma\left(\eta_{s}^{N}: s \leq t\right)$. To avoid unnecessary technical considerations, we assume throughout this article that there is no explosion.

Denote by $D\left(\mathbb{R}_{+}, E_{N}\right)$ the space of right-continuous trajectories $e: \mathbb{R}_{+} \rightarrow E_{N}$ with left limits endowed with the Skorohod topology. Let $\mathbf{P}_{\eta}^{N}, \eta \in E_{N}$, be the probability measure on $D\left(\mathbb{R}_{+}, E_{N}\right)$ induced by the Markov process $\left\{\eta_{t}^{N}: t \geq\right.$ $0\}$ starting from $\eta$. Expectation with respect to $\mathbf{P}_{\eta}^{N}$ is denoted by $\mathbf{E}_{\eta}^{N}$ and we frequently omit the index $N$ in $\mathbf{P}_{\eta}^{N}, \mathbf{E}_{\eta}^{N}$.

For every $N \geq 1$ and any subset $A \subseteq E_{N}$, denote by $\tau_{A}: D\left(\mathbb{R}_{+}, E_{N}\right) \rightarrow \mathbb{R}_{+}$the hitting time of the set $A$ :

$$
\tau_{A}:=\inf \left\{s>0: e_{s} \in A\right\},
$$

with the convention that $\tau_{A}=\infty$ if $e_{s} \notin A$ for all $s>0$. When the set $A$ is a singleton $\{\eta\}$, we denote $\tau_{\{\eta\}}$ by $\tau_{\eta}$. This convention is adopted everywhere below for any variable depending on a set. In addition, for each $t \geq 0$, define the additive functional $\mathcal{T}_{t}^{A}: D\left(\mathbb{R}_{+}, E\right) \mapsto \mathbb{R}_{+}$as the amount of time the process stayed in the set $A$ in the interval $[0, t]$ :

$$
\mathcal{T}_{t}^{A}:=\int_{0}^{t} \mathbf{1}\left\{e_{s} \in A\right\} d s, \quad t \geq 0,
$$

where $\mathbf{1}\{B\}$ stands for the indicator of the set $B$.

A sequence of states $\boldsymbol{\eta}=\left(\eta^{N} \in E_{N}: N \geq 1\right)$ is said to be a point in a sequence $\mathcal{A}$ of subsets of $E_{N}, \mathcal{A}=\left(A_{N} \subseteq E_{N}: N \geq 1\right)$, if $\eta^{N}$ belongs to $A_{N}$ for every $N \geq 1$. For a point $\boldsymbol{\eta}=\left(\eta^{N} \in E_{N}: N \geq 1\right)$ and a set $\mathcal{A}=\left(A_{N} \subseteq E_{N}: N \geq 1\right)$, denote by $T_{\boldsymbol{\eta}}, T_{\mathcal{A}}$, the hitting times of the sets $\{\boldsymbol{\eta}\}, \mathcal{A}$ :

$$
T_{\boldsymbol{\eta}}=T_{\boldsymbol{\eta}}^{N}:=\tau_{\eta^{N}}, \quad T_{\mathcal{A}}=T_{\mathcal{A}}^{N}:=\tau_{A_{N}} .
$$


For any sequence of subsets $\mathcal{A}=\left(A_{N} \subset E_{N}: N \geq 1\right), \mathcal{F}=\left(F_{N} \subset E_{N}: N \geq 1\right)$, denote by $T_{\mathcal{A}}(\mathcal{F})$ the time spent on the set $\mathcal{F}$ before hitting the set $\mathcal{A}$ :

$$
T_{\mathcal{A}}(\mathcal{F})=T_{\mathcal{A}}^{N}(\mathcal{F}):=\int_{0}^{\tau_{A_{N}}} \mathbf{1}\left\{\eta_{s}^{N} \in F_{N}\right\} d s .
$$

2.1. Valley with attractor. We introduce in this subsection the concept of valley. Intuitively, a subset $W$ of the state space $E_{N}$ is a valley for the Markov process $\left\{\eta_{t}^{N}: t \geq 0\right\}$ if the process starting from $W$ thermalizes in $W$ before leaving $W$ at an exponential random time.

To define precisely a valley, consider two sequences $\mathcal{W}, \mathcal{B}$ of subsets of $E_{N}$, the second one containing the first and being properly contained in $E_{N}$ :

$$
\mathcal{W}=\left(W_{N} \subseteq E_{N}: N \geq 1\right), \mathcal{B}=\left(B_{N} \subseteq E_{N}: N \geq 1\right), \quad W_{N} \subseteq B_{N} \varsubsetneqq E_{N} .
$$

Fix a point $\boldsymbol{\xi}=\left(\xi_{N} \in W_{N}: N \geq 1\right)$ in $\mathcal{W}$, a sequence of positive numbers $\boldsymbol{\theta}=\left(\theta_{N}: N \geq 1\right)$ and denote by $\mathcal{B}^{c}$ the complement of $\mathcal{B}: \mathcal{B}^{c}=\left(B_{N}^{c}: N \geq 1\right)$.

Definition 2.1 (Valley). The triple $(\mathcal{W}, \mathcal{B}, \boldsymbol{\xi})$ is a valley of depth $\boldsymbol{\theta}$ and attractor $\boldsymbol{\xi}$ for the Markov process $\left\{\eta_{t}^{N}: t \geq 0\right\}$ if for every point $\boldsymbol{\eta}=\left(\eta^{N}: N \geq 1\right)$ in $\mathcal{W}$

(V1) With overwhelming probability, the attractor $\boldsymbol{\xi}$ is attained before the process leaves $\mathcal{B}$ :

$$
\lim _{N \rightarrow \infty} \mathbf{P}_{\eta^{N}}\left[T_{\boldsymbol{\xi}}<T_{\mathcal{B}^{c}}\right]=1 ;
$$

(V2) The law of $T_{\mathcal{B}^{c}} / \theta_{N}$ under $\mathbf{P}_{\eta^{N}}$ converges to a mean 1 exponential distribution, as $N \rightarrow \infty$;

(V3) For every $\delta>0$,

$$
\lim _{N \rightarrow \infty} \mathbf{P}_{\eta^{N}}\left[\frac{1}{\theta_{N}} T_{\mathcal{B}^{c}}(\boldsymbol{\Delta})>\delta\right]=0,
$$

where $\boldsymbol{\Delta}=\left(\Delta_{N}: N \geq 1\right)$ and $\Delta_{N}$ is the annulus $B_{N} \backslash W_{N}$.

We refer to $\mathcal{W}$ as the well, and $\mathcal{B}$ as the basin of the valley $(\mathcal{W}, \mathcal{B}, \boldsymbol{\xi})$. We present in Section 3 examples of Markov processes on finite state spaces and triples $(\mathcal{W}, \mathcal{B}, \boldsymbol{\xi})$ in which all conditions but one in the above definition hold.

Condition (V1). The first condition guarantees that the process thermalizes in $\mathcal{W}$ before leaving the basin $\mathcal{B}$. We prove in Lemma 4.1 that conditions (V1), (V2) imply that the attractor $\boldsymbol{\xi}$ is reached from any point in the well $\mathcal{W}$ faster than $\theta_{N}$ :

$$
\lim _{N \rightarrow \infty} \sup _{\eta \in W_{N}} \mathbf{P}_{\eta}\left[\frac{1}{\theta_{N}} T_{\boldsymbol{\xi}}>\delta\right]=0 .
$$

Conversely, this condition and (V2) warrant the validity of (V1). We may therefore replace (V1) by (V1') in the definition.

Example 3.2 illustrates the fact that conditions (V2), (V3) may hold while (V1) fails. In this example, with overwhelming probability, the process, starting from one state in the well $\mathcal{W}$, leaves the basin $\mathcal{B}$ at an exponential time before hitting the attractor $\xi$.

Of course, the existence of an attractor is superfluous, as shown by Example 3.8. where we present a valley without an attractor. This requirement could be replaced by weaker requisites on the spectrum of the generator in the reversible case or on the total variation distance between the state of the process and the invariant measure restricted to the well $\mathcal{W}$. Nevertheless, in several non trivial 
examples, as in the case of condensed zero-range processes [3, 4, which motivated this paper, attractors do exist.

Condition (V2). The second condition asserts that the process leaves the basin at an exponential time of order given by the depth of the valley. Example 3.5presents a situation in which conditions (V1), (V3) hold but not (V2) nor (V1'). There, the order of magnitude of the time needed for the process to reach $\mathcal{B}^{c}$ from $\mathcal{W}$ depends on the starting point of $\mathcal{W}$.

Clearly, the depth of a valley is defined up to an equivalence relation: if $\boldsymbol{\theta}^{\prime}=\left(\theta_{N}^{\prime}\right.$ : $N \geq 1)$ is another sequence of positive numbers such that $\lim _{N \rightarrow \infty}\left(\theta_{N} / \theta_{N}^{\prime}\right)=1$, the valley has also depth $\boldsymbol{\theta}^{\prime}$. Moreover, the depth of a valley depends on the basin. As we shall see in Example 3.3. two different valleys $(\mathcal{W}, \mathcal{B}, \boldsymbol{\xi}),\left(\mathcal{W}, \mathcal{B}^{\prime}, \boldsymbol{\xi}\right)$, with $\mathcal{B} \subset \mathcal{B}^{\prime}$, may have depths of different order. Finally, the depth has not an intrinsic character, in contrast with valleys, in the sense that it changes if we speed up or slow down the underlying Markov process.

Condition (V3). The last condition requires the process starting from the well to spend a negligible amount of time in the part of the basin which does not belong to the well.

We prove in Lemma 4.2 that we may replace condition (V3) by the assumption that for every point $\boldsymbol{\eta}=\left(\eta^{N}: N \geq 1\right)$ in $\mathcal{W}$ and every $t>0$,

$$
\lim _{N \rightarrow \infty} \mathbf{E}_{\eta^{N}}\left[\int_{0}^{\min \left\{t, \theta_{N}^{-1} T_{\mathcal{B}^{c}}\right\}} \mathbf{1}\left\{\eta_{s \theta_{N}} \in \Delta_{N}\right\} d s\right]=0
$$

Condition (V3) is necessary, as we shall see in Example 3.1 to ensure that $\mathcal{W}$ is the well of the valley and not an evanescent set. The Markov process presented in this example fulfills conditions (V1), (V2) but not condition (V3).

The definition of valley focus on paths of the Markov process starting from the well $\mathcal{W}$. Nothing is imposed on the process starting from the annulus $\boldsymbol{\Delta}$, which may hide other wells, even deeper than the well $\mathcal{W}$, as illustrated by example 3.7 To rule out this eventuality, we replace condition (V3) by assumption (V3') which reads:

For every $\delta>0$,

$$
\lim _{N \rightarrow \infty} \sup _{\eta \in B_{N}} \mathbf{P}_{\eta}\left[\frac{1}{\theta_{N}} T_{\mathcal{B}^{c}}(\boldsymbol{\Delta})>\delta\right]=0 .
$$

Fix $\eta$ in $\Delta_{N}$ and note that $T_{\mathcal{W} \cup \mathcal{B}^{c}}=T_{\mathcal{W} \cup \mathcal{B}^{c}}(\boldsymbol{\Delta}) \leq T_{\mathcal{B}^{c}}(\boldsymbol{\Delta}) \mathbf{P}_{\eta}$ almost surely. Therefore, it follows from condition ( $\mathbf{V} 3$ ') that the process starting from $\boldsymbol{\Delta}$ immediately reaches $\mathcal{W} \cup \mathcal{B}^{c}$ : For every $\delta>0$,

$$
\lim _{N \rightarrow \infty} \sup _{\eta \in \Delta_{N}} \mathbf{P}_{\eta}\left[\frac{1}{\theta_{N}} T_{\mathcal{W} \cup \mathcal{B}^{c}}>\delta\right]=0
$$

It also follows from conditions (V2), (V3') that

$$
\lim _{N \rightarrow \infty} \sup _{\eta \in B_{N}} \mathbf{E}_{\eta}\left[\int_{0}^{\min \left\{t, \theta_{N}^{-1} T_{\mathcal{B} c}\right\}} \mathbf{1}\left\{\eta_{s \theta_{N}} \in \Delta_{N}\right\} d s\right]=0
$$

These remarks lead naturally to a more restrictive definition of valley. 
Definition 2.2 (S-Valley). The triple $(\mathcal{W}, \mathcal{B}, \boldsymbol{\xi})$ is a S-valley of depth $\boldsymbol{\theta}$ and attractor $\boldsymbol{\xi}$ for the Markov process $\left\{\eta_{t}^{N}: t \geq 0\right\}$ if, for every point $\boldsymbol{\eta}=\left(\eta^{N}: N \geq 1\right)$ in $\mathcal{W}$, assumptions (V1), (V2), (V3') are fulfilled.

In Example 3.7 we present a triple $(\mathcal{W}, \mathcal{B}, \boldsymbol{\xi})$ satisfying assumptions (V1), (V2), (V3) but not $(\mathbf{V 3}),(2.5)$ and (2.6) because $\boldsymbol{\Delta}$ contains a well deeper than $\mathcal{W}$.

In many cases, it is possible to transfer from $\boldsymbol{\Delta}$ to $\mathcal{B}^{c}$ all points in $\boldsymbol{\Delta}$ which do not reach immediately $\mathcal{W} \cup \mathcal{B}^{c}$, in the sense of condition (2.5), to obtain from a valley $(\mathcal{W}, \mathcal{B}, \boldsymbol{\xi})$ satisfying conditions $(\mathbf{V 1}),(\mathbf{V} 2),(\mathbf{V} \mathbf{3})$ a new valley $\left(\mathcal{W}, \mathcal{B}^{\prime}, \boldsymbol{\xi}\right)$ satisfying conditions (V1), (V2), (V3'). We refer to Example 3.7.

We present in Example 3.4 a triple which satisfies conditions (V1), (V2), (2.5) but not (V3). In particular, the first three conditions do not imply (V3). In this example, there is a state in the annulus $\Delta_{N}$ which immediately jumps to the well $W_{N}$, but which is visited several times before leaving the basin $B_{N}$.

2.2. Tunneling and Metastability. Given a sequence of Markov processes $\left\{\eta_{t}^{N}\right.$ : $t \geq 0\}$ with values in $E_{N}$, we might observe a complex landscape of valleys with a wide variety of depths. We describe in this subsection the inter-valley dynamics.

Fix a finite number of disjoint subsets $\mathcal{E}_{N}^{1}, \ldots, \mathcal{E}_{N}^{\kappa}, \kappa \geq 2$, of $E_{N}: \mathcal{E}_{N}^{x} \cap \mathcal{E}_{N}^{y}=\varnothing$, $x \neq y$. Let $\mathcal{E}_{N}=\cup_{x \in S} \mathcal{E}_{N}^{x}$ and let $\Delta_{N}=E_{N} \backslash \mathcal{E}_{N}$ so that

$$
E_{N}=\underbrace{\varepsilon_{N}^{1} \cup \cdots \cup \mathcal{E}_{N}^{\kappa}}_{\varepsilon_{N}} \cup \Delta_{N} .
$$

Denote by $\Psi_{N}: \mathcal{E}_{N} \mapsto S=\{1,2, \ldots, \kappa\}$, the projection given by

$$
\Psi_{N}(\eta)=\sum_{x \in S} x \mathbf{1}\left\{\eta \in \mathcal{E}_{N}^{x}\right\}
$$

and let

$$
\breve{\mathcal{E}}_{N}^{x}:=\mathcal{E}_{N} \backslash \mathcal{E}_{N}^{x}, \quad \mathcal{E}^{x}=\left(\mathcal{E}_{N}^{x}: N \geq 1\right) \quad \text { and } \quad \breve{\mathcal{E}}^{x}=\left(\breve{\mathcal{E}}_{N}^{x}: N \geq 1\right) .
$$

For a subset $A$ of $E_{N}$, let $\mathcal{S}_{t}^{A}$ be the generalized inverse of the additive functional $\mathcal{T}_{t}^{A}$ introduced in the beginning of this section:

$$
\mathcal{S}_{t}^{A}(e .):=\sup \left\{s \geq 0: \mathcal{T}_{s}^{A}(e .) \leq t\right\} .
$$

It is clear that $\mathcal{S}_{t}^{A}<+\infty$ for every $t \geq 0$ if, and only if, $\mathcal{T}_{t}^{A} \rightarrow+\infty$ as $t \rightarrow+\infty$. To circumvent the case $\mathcal{S}_{t}^{A}=\infty$, add an artificial point $\mathfrak{d}$ to the subset $A$. For any path $e . \in D\left(\mathbb{R}_{+}, E_{N}\right)$ starting at $e_{0} \in A$, denote by $e^{A}$ the trace of the path $e$. on the set $A$ defined by $e_{t}^{A}=e_{S_{t}^{A}}$ if $S_{t}^{A}<+\infty$, and $e_{t}^{A}=\mathfrak{d}$ otherwise. Clearly, if $e_{t}^{A}=\mathfrak{d}$ for some $t$, then $e_{s}^{A}=\mathfrak{d}$, for every $s>t$.

Denote by $\left\{\eta_{t}^{\mathcal{E}_{N}}: t \geq 0\right\}$ the $\mathcal{E}_{N} \cup\{\mathfrak{d}\}$-valued Markov process obtained as the trace of $\left\{\eta_{t}: t \geq 0\right\}$ on $\mathcal{E}_{N}$, and by $\left\{X_{t}^{N}: t \geq 0\right\}$ the stochastic process defined by $X_{t}^{N}=\Psi_{N}\left(\eta_{t}^{\mathcal{E}_{N}}\right)$ whenever $\eta_{t}^{\mathcal{E}_{N}} \in \mathcal{E}_{N}$ and $X_{t}^{N}=\mathfrak{d}$ otherwise. Clearly, besides trivial cases, $X^{N}$ is not Markovian.

Let $\boldsymbol{\theta}=\left(\theta_{N}: N \geq 1\right)$ denote a sequence of positive numbers and, for each $x \in S$, let $\boldsymbol{\xi}_{x}=\left(\xi_{x}^{N}: N \geq 1\right)$ be a point in $\mathcal{E}^{x}$. In order to describe the asymptotic behaviour of the Markov process on the time-scale $\boldsymbol{\theta}$ we use a Markov process $\left\{\mathbb{P}_{x}: x \in S\right\}$ defined on the canonical path space $D\left(\mathbb{R}_{+}, S\right)$.

Definition 2.3 (Tunneling). A sequence of Markov processes $\left\{\eta_{t}^{N}: t \geq 0\right\}, N \geq 1$, on a countable state space $E=\left(E_{N}: N \geq 1\right)$ exhibits a tunneling behaviour on the 
time-scale $\boldsymbol{\theta}$, with metastates $\left\{\mathcal{E}^{x}: x \in S\right\}$, metapoints $\left\{\boldsymbol{\xi}_{x}: x \in S\right\}$ and asymptotic Markov dynamics $\left\{\mathbb{P}_{x}: x \in S\right\}$ if, for each $x \in S$,

(M1) The point $\boldsymbol{\xi}_{x}$ is an attractor on $\mathcal{E}^{x}$ in the sense that

$$
\lim _{N \rightarrow \infty} \inf _{\eta \in \mathcal{E}_{N}^{x}} \mathbf{P}_{\eta}\left[T_{\boldsymbol{\xi}_{x}}<T_{\breve{\varepsilon}^{x}}\right]=1
$$

(M2) For every point $\boldsymbol{\eta}=\left(\eta^{N}: N \geq 1\right)$ in $\mathcal{E}^{x}$, the law of the speeded up process $\left\{X_{t \theta_{N}}^{N}: t \geq 0\right\}$ under $\mathbf{P}_{\eta^{N}}$ converges to $\mathbb{P}_{x}$ as $N \uparrow \infty$;

(M3) For every $t>0$,

$$
\lim _{N \rightarrow+\infty} \sup _{\eta \in \mathcal{E}_{N}^{x}} \mathbf{E}_{\eta}\left[\int_{0}^{t} \mathbf{1}\left\{\eta_{s \theta_{N}}^{N} \in \Delta_{N}\right\} d s\right]=0 .
$$

Let $\boldsymbol{\Delta}$ denote the sequence $\left(\Delta_{N}: N \geq 1\right)$ and consider the triple $\left(\mathcal{E}^{x}, \mathcal{E}^{x} \cup \boldsymbol{\Delta}, \boldsymbol{\xi}_{x}\right)$ for a fixed $x$ in $S$. Clearly, if $x$ is not an absorbing state for the asymptotic Markov dynamics, the triple $\left(\mathcal{E}^{x}, \mathcal{E}^{x} \cup \boldsymbol{\Delta}, \boldsymbol{\xi}_{x}\right)$ is a valley of depth of the order of $\boldsymbol{\theta}$. In this case, it may happen that the triple $\left(\mathcal{E}^{x}, \mathcal{E}^{x} \cup \boldsymbol{\Delta}, \xi_{x}\right)$ is an inaccessible valley in the sense that once the process escapes from $\mathcal{E}^{x}$ it never returns to $\mathcal{E}^{x}$. This is illustrated in Example 3.6. In contrast, if $x$ is an absorbing state for the asymptotic Markov dynamics not much information is available on the triple $\left(\mathcal{E}^{x}, \mathcal{E}^{x} \cup \boldsymbol{\Delta}, \boldsymbol{\xi}_{x}\right)$. Example 3.5 presents a Markov process which exhibits a tunneling behavior in which a triple is not a valley. In this example the triple contains a well of larger order depth than $\boldsymbol{\theta}$.

Suppose that property (M2) is satisfied for a sequence of Markov processes and denote by $S_{*} \subset S$ the subset of non-absorbing states for $\left\{\mathbb{P}_{x}: x \in S\right\}$. For the states in $S_{*}$ we may replace requirement (M3) by property (V3) of valley, namely: For each $x \in S_{*}$,

$$
\lim _{N \rightarrow \infty} \sup _{\eta \in \varepsilon_{N}^{x}} \mathbf{P}_{\eta}\left[\frac{1}{\theta_{N}} T_{\breve{\varepsilon}^{x}}(\boldsymbol{\Delta})>\delta\right]=0 .
$$

Proposition 2.4. Assume that (M2) is fulfilled for a sequence of Markov processes $\left\{\eta_{t}^{N}: t \geq 0\right\}, N \geq 1$. If (M3) is satisfied for each $x \in S \backslash S_{*}$ and if (C1) holds for any $x \in S_{*}$, then (M3) is in force for any $x \in S$.

We arrive to the same conclusion in Proposition 2.4 if we assume instead that (C1) holds for every state $x \in S$. This is the content of Lemma 4.7. Actually, for an absorbing state $x$, property $(\mathbf{C 1})$ is stronger than (M3) because in this case $\theta_{N}^{-1} T_{\breve{\varepsilon}^{x}}$ diverges.

The definition of tunneling examines the inter-valley dynamics between wells with depths of the same order. It is far from a global description since it does not exclude the possibility that $\boldsymbol{\Delta}$ contains a landscape of valleys of depths of larger order than $\boldsymbol{\theta}$. This situation is illustrated in Example 3.7. We have also just seen that if $x$ is an absorbing state for the asymptotic Markov dynamics, the set $\mathcal{E}^{x}$ may also contain a landscape of valleys of larger order depth. In order to exclude these eventualities, we impose more restrictive conditions in the definition of metastability. We replace (M1) by (M1') to ensure that there are no wells in $\mathcal{E}^{x}$ of depth of order $\theta_{N}$ if $x$ is an absorbing point for the asymptotic Markov dynamics; and we replace (M3) by (M3') to avoid wells in $\boldsymbol{\Delta}$ of depth of order $\theta_{N}$ or larger.

Definition 2.5 (Metastability). A sequence of Markov processes $\left\{\eta_{t}^{N}: t \geq 0\right\}$, $N \geq 1$, on a countable state space $E=\left(E_{N}: N \geq 1\right)$ exhibits a metastable behaviour 
on the time-scale $\boldsymbol{\theta}$, with metastates $\left\{\mathcal{E}^{x}: x \in S\right\}$, metapoints $\left\{\boldsymbol{\xi}_{x}: x \in S\right\}$ and asymptotic Markov dynamics $\left\{\mathbb{P}_{x}: x \in S\right\}$, if for each $x \in S$,

(M1') The point $\boldsymbol{\xi}_{x}$ is an attractor on $\mathcal{E}^{x}$ in the sense that for every $\delta>0$

$$
\lim _{N \rightarrow \infty} \sup _{\eta \in \mathcal{E}_{N}^{x}} \mathbf{P}_{\eta}\left[T_{\boldsymbol{\xi}_{x}}>\delta \theta_{N}\right]=0 ;
$$

(M2) For every point $\boldsymbol{\eta}=\left(\eta^{N}: N \geq 1\right)$ in $\mathcal{E}^{x}$, the law of the speeded up process $\left\{X_{t \theta_{N}}^{N}: t \geq 0\right\}$ under $\mathbf{P}_{\eta^{N}}$ converges to $\mathbb{P}_{x}$ as $N \uparrow \infty$;

(M3') For every $t>0$,

$$
\lim _{N \rightarrow+\infty} \sup _{\eta \in E_{N}} \mathbf{E}_{\eta}\left[\int_{0}^{t} \mathbf{1}\left\{\eta_{s \theta_{N}}^{N} \in \Delta_{N}\right\} d s\right]=0 .
$$

As for valleys, it follows from (M3') that $\boldsymbol{\Delta}$ is evanescent in the sense that for every $\delta>0$,

$$
\lim _{N \rightarrow \infty} \sup _{\eta \in \Delta_{N}} \mathbf{P}_{\eta}\left[T_{\varepsilon_{N}}>\delta \theta_{N}\right]=0 .
$$

Example 3.5 presents a Markov process which exhibits a tunneling behaviour and fulfills condition (M3') but violates assumption (M1'). Example 3.7 presents a Markov process with the opposite properties. It fulfills conditions (M1'), (M2), (M3) but violates assumption (M3'). This latter example is very instructive. It shows that the same Markov process may have distinct metastable behaviors at different time scales. This occurs when on one time scale there is an isolated point in the asymptotic Markov dynamics. In longer time scales this metastate is reached by other metastates, previous metastates coalesce in one larger metastate, and a new metastable picture emerges.

We conclude this subsection observing that we may define metastability without referring to trace processes. Indeed, consider the $S$-valued stochastic process $\hat{X}_{t}^{N}$ defined as

$$
\hat{X}_{t}^{N}=\Psi_{N}\left(\eta_{\sigma(t)}^{N}\right),
$$

where $\sigma(t):=\sup \left\{s \leq t: \eta_{s}^{N} \in \mathcal{E}_{N}\right\}$. Note that $\hat{X}_{t}^{N}$ is well defined whenever $\eta_{t}^{N}$ starts from a point in $\mathcal{E}_{N}$.

Proposition 2.6. In condition (M2) of Definitions 2.3, 2.5, we may replace the stochastic process $\left\{X_{t \theta_{N}}^{N}: t \geq 0\right\}$ by $\left\{\hat{X}_{t \theta_{N}}^{N}: t \geq 0\right\}$.

2.3. The positive recurrent case. The purpose of this subsection is to provide sufficient conditions to ensure tunneling. Assume from now on that the Markov process $\left\{\eta_{t}^{N}: t \geq 0\right\}$ is irreducible and positive recurrent, and denote by $\mu_{N}$ its unique invariant probability measure. It follows from these hypotheses that the holding rates $\lambda^{N}(\eta)=-R_{N}(\eta, \eta)$ are strictly positive, and that the discrete time Markov chain on $E_{N}$ which jumps from $\eta$ to $\xi$ at rate $R_{N}(\eta, \xi) / \lambda^{N}(\eta)$ is irreducible and recurrent.

Furthermore, for every $\eta \in E_{N}$ and $A \subseteq E_{N}, \mathcal{T}_{t}^{A}$ diverges. Consequently, the trace of the Markov process $\left\{\eta_{t}^{N}: t \geq 0\right\}$ on the set $A$, denoted by $\left\{\eta_{t}^{A}: t \geq 0\right\}$, is well defined and takes values in $A$. In fact, we prove in Proposition 6.1 that the trace $\left\{\eta_{t}^{A}: t \geq 0\right\}$ is an irreducible and positive recurrent Markov process with invariant probability measure equal to the measure $\mu_{N}$ conditioned on the set $A$. 
Consider two sequences of sets $\mathcal{W}$ and $\mathcal{B}$ satisfying (2.3). To keep notation simple, let $\Delta_{N}=B_{N} \backslash W_{N}, \boldsymbol{\Delta}=\left(\Delta_{N}: N \geq 1\right)$, and $\mathcal{E}_{N}=W_{N} \cup B_{N}^{c}, \mathcal{E}=\left(\mathcal{E}_{N}: N \geq 1\right)$. Denote by

$$
R_{N}^{\varepsilon}(\eta, \xi), \quad \eta, \xi \in \mathcal{E}_{N}, \eta \neq \xi
$$

the transition rates of the Markov process $\left\{\eta_{t}^{\mathcal{E}_{N}}: t \geq 0\right\}$, the trace of $\left\{\eta_{t}^{N}: t \geq 0\right\}$ on $\mathcal{E}_{N}$. Let $R_{N}^{\mathcal{W}}: W_{N} \rightarrow \mathbb{R}_{+}$be the rate at which the trace process jumps to $B_{N}^{c}$ :

$$
R_{N}^{\mathcal{W}}(\eta):=\sum_{\xi \in B_{N}^{c}} R_{N}^{\varepsilon}(\eta, \xi)
$$

and let $r_{N}\left(\mathcal{W}, \mathcal{B}^{c}\right)$ be the average of $R_{N}^{\mathcal{W}}$ over $W_{N}$ with respect to $\mu_{N}^{W_{N}}$, the measure $\mu_{N}$ conditioned on $W_{N}$ :

$$
\begin{aligned}
r_{N}\left(\mathcal{W}, \mathcal{B}^{c}\right) & :=\frac{1}{\mu_{N}\left(W_{N}\right)} \sum_{\eta \in W_{N}} R_{N}^{\mathcal{W}}(\eta) \mu_{N}(\eta) \\
& =\frac{1}{\mu_{N}\left(W_{N}\right)} \sum_{\eta \in W_{N}} \sum_{\xi \in B_{N}^{c}} R_{N}^{\varepsilon}(\eta, \xi) \mu_{N}(\eta) .
\end{aligned}
$$

Next theorem presents sufficient conditions for $\mathcal{W}$ and $\mathcal{B}$ to be the well and the basin of a valley.

Theorem 2.7. Assume that there exists a point $\boldsymbol{\xi}=\left(\xi^{N}: N \geq 1\right)$ in $\mathcal{W}$ such that for every point $\boldsymbol{\eta}=\left(\eta^{N}: N \geq 1\right)$ in $\mathcal{W}$,

$$
\begin{gathered}
\lim _{N \rightarrow \infty} \mathbf{E}_{\eta^{N}}\left[\int_{0}^{T_{\xi}} R_{N}^{\mathcal{W}}\left(\eta_{s}^{N}\right) \mathbf{1}\left\{\eta_{s}^{N} \in W_{N}\right\} d s\right]=0, \\
\lim _{N \rightarrow \infty} r_{N}\left(\mathcal{W}, \mathcal{B}^{c}\right) \mathbf{E}_{\eta^{N}}\left[T_{\boldsymbol{\xi}}(\mathcal{W})\right]=0
\end{gathered}
$$

and

$$
\lim _{N \rightarrow \infty} r_{N}\left(\mathcal{W}, \mathcal{B}^{c}\right) \mathbf{E}_{\eta^{N}}\left[T_{\mathcal{B}^{c}}(\boldsymbol{\Delta})\right]=0
$$

Then, $(\mathcal{W}, \mathcal{B}, \boldsymbol{\xi})$ is a valley with depth $\boldsymbol{\theta}=\left(\theta_{N}: N \geq 1\right)$ where $\theta_{N}=1 / r_{N}\left(\mathcal{W}, \mathcal{B}^{c}\right)$, $N \geq 1$.

Conditions (2.9) and (2.10) clearly follow from the stronger condition

$$
\lim _{N \rightarrow \infty} \sup \left\{R_{N}^{\mathcal{W}}(\eta): \eta \in W_{N}\right\} \mathbf{E}_{\eta^{N}}\left[T_{\boldsymbol{\xi}}\right]=0 .
$$

To state sufficient conditions for a tunneling behaviour, recall the notation introduced in Subsection 2.2. Let $R_{N}^{\mathcal{E}}: \mathcal{E}_{N} \times \mathcal{E}_{N} \rightarrow \mathbb{R}_{+}$be the transition rates of the trace process $\left\{\eta_{t}^{\mathcal{E}_{N}}: t \geq 0\right\}$, let $R_{N}^{x, y}: \mathcal{E}_{N}^{x} \rightarrow \mathbb{R}_{+}, x, y \in S, x \neq y$, be the rate at which the trace process jumps to the set $\mathcal{E}_{N}^{y}$ :

$$
R_{N}^{x, y}(\eta):=\sum_{\xi \in \mathcal{E}_{N}^{y}} R_{N}^{\varepsilon}(\eta, \xi)
$$

and let $R_{N}^{x}: \mathcal{E}_{N}^{x} \rightarrow \mathbb{R}_{+}, x \in S$, be the rate at which it jumps to the set $\breve{\mathcal{E}}_{N}^{x}: R_{N}^{x}=$ $\sum_{y \neq x} R_{N}^{x, y}$. Observe that $R_{N}^{x}$ coincides with $R_{N}^{\mathcal{W}}$ if $\left(W_{N}, B_{N}\right)=\left(\mathcal{E}_{N}^{x}, \mathcal{E}_{N}^{x} \cup \Delta_{N}\right)$.

Let $\mu_{N}^{x}$ stand for the probability measure $\mu_{N}$ conditioned on $\mathcal{E}_{N}^{x}$. Denote by $r_{N}\left(\mathcal{E}^{x}, \mathcal{E}^{y}\right)$ the $\mu_{N}^{x}$-expectation of $R_{N}^{x, y}$ :

$$
r_{N}\left(\mathcal{E}^{x}, \mathcal{E}^{y}\right):=\frac{1}{\mu_{N}\left(\mathcal{E}_{N}^{x}\right)} \sum_{\eta \in \mathcal{E}_{N}^{x}} R_{N}^{x, y}(\eta) \mu_{N}(\eta)
$$


and by $r_{N}\left(\mathcal{E}^{x}, \breve{\varepsilon}_{N}^{x}\right)$ the $\mu_{N}^{x}$-expectation of $R_{N}^{x}$ so that

$$
r_{N}\left(\mathcal{E}^{x}, \breve{\varepsilon}_{N}^{x}\right)=\sum_{y \neq x} r_{N}\left(\mathcal{E}^{x}, \mathcal{E}^{y}\right) .
$$

To guarantee that the process $\left\{\eta_{t}^{N}: t \geq 0\right\}$ exhibits a tunneling behavior, we first require that each subset $\left\{\mathcal{E}^{x}: x \in S\right\}$ satisfies conditions (2.9) and (2.10): For each $x \in S$, there exists a point $\boldsymbol{\xi}_{x}=\left(\xi_{x}^{N}: N \geq 1\right)$ in $\mathcal{E}^{x}$ such that

$$
\lim _{N \rightarrow \infty} \mathbf{E}_{\eta^{N}}\left[\int_{0}^{T_{\xi_{x}}} R_{N}^{x}\left(\eta_{s}^{N}\right) \mathbf{1}\left\{\eta_{s}^{N} \in \mathcal{E}_{N}^{x}\right\} d s\right]=0
$$

and

$$
\lim _{N \rightarrow \infty} r_{N}\left(\mathcal{E}^{x}, \breve{\mathcal{E}}^{x}\right) \mathbf{E}_{\eta^{N}}\left[T_{\boldsymbol{\xi}_{x}}\left(\mathcal{E}^{x}\right)\right]=0
$$

for every point $\boldsymbol{\eta}=\left(\eta^{N}: N \geq 1\right)$ in $\mathcal{E}^{x}$.

Theorem 2.8. Suppose (C2), (C3) and that there exists a sequence $\boldsymbol{\theta}=\left(\theta_{N}\right.$ : $N \geq 1$ ) of positive numbers such that, for every pair $x, y \in S, x \neq y$, the following limit exists

$$
r(x, y):=\lim _{N \rightarrow \infty} \theta_{N} r_{N}\left(\mathcal{E}^{x}, \mathcal{E}^{y}\right) .
$$

Assume, furthermore, that (M3) is satisfied for each absorbing state $x$ of the Markov process on $S$ determined by the rates $r$ and that $(\mathbf{C 1})$ holds for any nonabsorbing state. Then, the sequence of Markov processes $\left\{\eta_{t}^{N}: t \geq 0\right\}, N \geq 1$, exhibits a tunneling behaviour on the time-scale $\boldsymbol{\theta}$, with metastates $\left\{\mathcal{E}^{x}: x \in S\right\}$, metapoints $\left\{\boldsymbol{\xi}_{x}: x \in S\right\}$ and asymptotic Markov dynamics characterized by the rates $r(x, y), x, y \in S$.

Notice that in the previous theorem we might get

$$
\sum_{x \in S \backslash\left\{x_{0}\right\}} r\left(x, x_{0}\right)=0 \text { and } \sum_{x \in S \backslash\left\{x_{0}\right\}} r\left(x_{0}, x\right)>0
$$

for some $x_{0} \in S_{*}$. In this case, the triple $\left(\mathcal{E}^{x_{0}}, \mathcal{E}^{x_{0}} \cup \boldsymbol{\Delta}, \boldsymbol{\xi}_{x_{0}}\right)$ turns out to be an inaccessible valley, as it is illustrated in Example 3.6. even tough it has the same depth than all the other wells involved in the tunneling.

2.4. The reversible case, potential theory. In addition to the positive recurrent assumption, let us now further assume that $\mu_{N}$ is a reversible probability measure. In this case, we may list simple conditions, all of them expressed in terms of the capacities and the reversible measure $\mu_{N}$, which ensure the existence of valleys and the tunneling behaviour.

As we have already seen, we need good estimates for the mean of entry times. In the reversible case, the mean of an entry time has a simple expression involving capacities, which are defined as follows. For two disjoint subsets $A, B$ of $E_{N}$ define

$$
\mathcal{C}_{N}(A, B):=\left\{f \in L^{2}\left(\mu_{N}\right): f(\eta)=1 \forall \eta \in A \text { and } f(\xi)=0 \forall \xi \in B\right\} .
$$

Let $\langle\cdot, \cdot\rangle_{\mu_{N}}$ stand for the scalar product in $L^{2}\left(\mu_{N}\right)$. Denote by $D_{N}$ the Dirichlet form associated to the generator $L_{N}$ :

$$
D_{N}(f):=\left\langle-L_{N} f, f\right\rangle_{\mu_{N}},
$$

for every $f$ in $L^{2}\left(\mu_{N}\right)$. An elementary computation shows that

$$
D_{N}(f)=\frac{1}{2} \sum_{\eta, \xi \in E_{N}} \mu_{N}(\eta) R_{N}(\eta, \xi)\{f(\xi)-f(\eta)\}^{2} .
$$


The capacity of two disjoint subsets $A, B$ of $E_{N}$ is defined as

$$
\operatorname{cap}_{N}(A, B):=\inf \left\{D_{N}(f): f \in \mathcal{C}_{N}(A, B)\right\} .
$$

In the reversible context, the expressions appearing in Theorems 2.7 and 2.8 can be computed by using capacities. Denote by $f_{A B}^{N}: E_{N} \rightarrow \mathbb{R}$ the function in $\mathcal{C}_{N}(A, B)$ defined as

$$
f_{A B}^{N}(\eta):=\mathbf{P}_{\eta}\left[\tau_{A}<\tau_{B}\right]
$$

In addition, for two points $\boldsymbol{\xi}=\left(\xi^{N}: N \geq 1\right)$ and $\boldsymbol{\eta}=\left(\eta^{N}: N \geq 1\right)$ in $\mathcal{W}, \eta^{N} \neq \xi^{N}$, $N \geq 1$, set $f_{N}(\boldsymbol{\eta}, \boldsymbol{\xi})=f_{\left\{\eta^{N}\right\}\left\{\xi^{N}\right\}}^{N}$ and $\operatorname{cap}_{N}(\boldsymbol{\eta}, \boldsymbol{\xi})=\operatorname{cap}_{N}\left(\left\{\eta^{N}\right\},\left\{\xi^{N}\right\}\right)$.

Consider two sequences of sets $\mathcal{W}$ and $\mathcal{B}$ satisfying (2.3) and recall the notation introduced in the previous subsection. By (6.16),

$$
\begin{gathered}
\mathbf{E}_{\eta^{N}}\left[\int_{0}^{T_{\xi}} R_{N}^{\mathcal{W}}\left(\eta_{s}^{N}\right) \mathbf{1}\left\{\eta_{s}^{N} \in W_{N}\right\} d s\right]=\frac{\left\langle R_{N}^{\mathcal{W}} \mathbf{1}\left\{W_{N}\right\}, f_{N}(\boldsymbol{\eta}, \boldsymbol{\xi})\right\rangle_{\mu_{N}}}{\operatorname{cap}_{N}(\boldsymbol{\eta}, \boldsymbol{\xi})}, \\
\mathbf{E}_{\eta^{N}}\left[T_{\boldsymbol{\xi}}(\mathcal{W})\right]=\frac{\left\langle\mathbf{1}\left\{W_{N}\right\}, f_{N}(\boldsymbol{\eta}, \boldsymbol{\xi})\right\rangle_{\mu_{N}}}{\operatorname{cap}_{N}(\boldsymbol{\eta}, \boldsymbol{\xi})}
\end{gathered}
$$

and, by Lemma 6.7

$$
r_{N}\left(\mathcal{W}, \mathcal{B}^{c}\right)=\frac{\operatorname{cap}_{N}\left(\mathcal{W}, \mathcal{B}^{c}\right)}{\mu_{N}(\mathcal{W})}
$$

In the last identity $\operatorname{cap}_{N}\left(\mathcal{W}, \mathcal{B}^{c}\right):=\operatorname{cap}_{N}\left(W_{N}, B_{N}^{c}\right)$ and $\mu_{N}(\mathcal{W}):=\mu_{N}\left(W_{N}\right)$. The previous relations can be used to check conditions (2.9) and (2.10) in Theorem 2.7 as well as assumptions $(\mathbf{C 2})$ and $(\mathbf{C 3})$ in Theorem 2.8.

Furthermore, since $0 \leq f_{N}(\boldsymbol{\eta}, \boldsymbol{\xi}) \leq 1$, and since, by (2.8), $\left\langle R_{N}^{\mathcal{W}} \mathbf{1}\left\{W_{N}\right\}\right\rangle_{\mu_{N}}=$ $\mu_{N}(\mathcal{W}) r_{N}\left(\mathcal{W}, \mathcal{B}^{c}\right)$, by (2.14),

$$
\begin{gathered}
\mathbf{E}_{\eta^{N}}\left[\int_{0}^{T_{\xi}} R_{N}^{\mathcal{W}}\left(\eta_{s}^{N}\right) \mathbf{1}\left\{\eta_{s}^{N} \in W_{N}\right\} d s\right] \leq \frac{\operatorname{cap}_{N}\left(\mathcal{W}, \mathcal{B}^{c}\right)}{\operatorname{cap}_{N}(\boldsymbol{\xi})} \\
\text { and } r_{N}\left(\mathcal{W}, \mathcal{B}^{c}\right) \mathbf{E}_{\eta^{N}}\left[T_{\boldsymbol{\xi}}(\mathcal{W})\right] \leq \frac{\operatorname{cap}_{N}\left(\mathcal{W}, \mathcal{B}^{c}\right)}{\operatorname{cap}_{N}(\boldsymbol{\xi})}
\end{gathered}
$$

where $\operatorname{cap}_{N}(\boldsymbol{\xi})=\inf \left\{\operatorname{cap}_{N}\left(\eta, \xi^{N}\right): \eta \in \mathcal{W} \backslash\left\{\xi^{N}\right\}\right\}$. Hence, conditions (2.9) and (2.10) follow from the stronger condition

$$
\lim _{N \rightarrow \infty} \frac{\operatorname{cap}_{N}\left(\mathcal{W}, \mathcal{B}^{c}\right)}{\operatorname{cap}_{N}(\boldsymbol{\xi})}=0
$$

Theorem 2.9. Assume that (2.15) holds for some point $\boldsymbol{\xi}=\left(\xi^{N}: N \geq 1\right)$ in $\mathcal{W}$ and that

$$
\lim _{N \rightarrow \infty} \frac{\mu_{N}\left(B_{N} \backslash W_{N}\right)}{\mu_{N}\left(W_{N}\right)}=0 .
$$

Then, for all points $\boldsymbol{\zeta}$ in $\mathcal{W},(\mathcal{W}, \mathcal{B}, \boldsymbol{\zeta})$ is a valley of depth $\mu_{N}(\mathcal{W}) / \operatorname{cap}_{N}\left(\mathcal{W}, \mathcal{B}^{c}\right)$, $N \geq 1$.

Assumption (2.15) is also powerful in the context of tunneling. Recall the notation introduced at the beginning of Subsection 2.2.

Theorem 2.10. Suppose that for each $x \in S$, there exists a point $\boldsymbol{\xi}_{x}=\left(\xi_{x}^{N}: N \geq\right.$ 1) in $\mathcal{E}^{x}$ such that

$$
\lim _{N \rightarrow \infty} \frac{\operatorname{cap}_{N}\left(\mathcal{E}^{x}, \breve{\mathcal{E}}^{x}\right)}{\operatorname{cap}_{N}\left(\boldsymbol{\xi}_{x}\right)}=0
$$


Suppose, furthermore, that (H0) holds for some $\boldsymbol{\theta}=\left(\theta_{N}: N \geq 1\right)$, that (M3) holds for each absorbing state of the Markov dynamics on $S$ determined by the rates $r$ and that

$$
\lim _{N \rightarrow \infty} \frac{\mu_{N}(\boldsymbol{\Delta})}{\mu_{N}\left(\mathcal{E}^{x}\right)}=0
$$

for each non-absorbing state $x$. Then, the sequence of Markov processes $\left\{\eta_{t}^{N}: t \geq\right.$ $0\}, N \geq 1$, exhibits a tunneling behaviour on the time-scale $\boldsymbol{\theta}$, with metastates $\left\{\mathcal{E}^{x}\right.$ : $x \in S\}$, metapoints $\left\{\boldsymbol{\xi}_{x}: x \in S\right\}$ and asymptotic Markov dynamics characterized by the rates $r(x, y), x, y \in S$.

Remark 2.11. In the previous theorem, we may replace condition (M3) for absorbing states and condition (H2) for non-absorbing states by the assumption

$$
\lim _{N \rightarrow \infty} \frac{1}{\theta_{N} r_{N}\left(\mathcal{E}^{x}, \breve{\mathcal{E}}^{x}\right)} \frac{\mu_{N}(\boldsymbol{\Delta})}{\mu_{N}\left(\mathcal{E}^{x}\right)}=0
$$

for all states $x$.

Note that condition (H2) and (H2') are equivalent for non-absorbing states if (H0) holds. This latter condition can be expressed in terms of capacities since, by Lemma 6.8. $\mu_{N}\left(\mathcal{E}^{x}\right) r_{N}\left(\mathcal{E}^{x}, \mathcal{E}^{y}\right)$ can be written as

$$
\frac{1}{2}\left\{\operatorname{cap}_{N}\left(\mathcal{E}^{x}, \breve{\mathcal{E}}^{x}\right)+\operatorname{cap}_{N}\left(\mathcal{E}^{y}, \breve{\mathcal{E}}^{y}\right)-\operatorname{cap}_{N}\left(\mathcal{E}^{x} \cup \mathcal{E}^{y}, \mathcal{E} \backslash\left(\mathcal{E}^{x} \cup \mathcal{E}^{y}\right)\right)\right\}
$$

for every $x, y \in S, x \neq y$.

One of the main steps in the proof of metastability is the replacement result presented in Lemma 6.4 and in Corollary 6.5. This statement proposes a mathematical formulation of the notion of thermalization by identifying this phenomenon with the possibility of replacing the time integral of a function by the time integral of its conditional expectation with respect to the $\sigma$-algebra generated by the metastable states. The existence of attractors allows a simple estimate, presented in Corollary 6.5. which plays a key role in all proofs.

The propositions stated above are proved in Section 4, while the theorems and the remark are proved in Section 5 .

\section{Some EXAMPLes}

We present in this section some examples to justify the definitions of the previous section and to illustrate some unexpected phenomena which may occur.

We start with a general remark concerning valleys on fixed state spaces. Consider a sequence of Markov processes $\left\{\eta_{t}^{N}: t \geq 0\right\}$ on some given countable space $E$ with generator $L_{N}$ described by (2.1). Denote by $\lambda_{N}(\eta)=\sum_{\xi \neq \eta} R_{N}(\eta, \xi)$ the rate at which the process leaves the state $\eta$. Clearly, the triple $(\{\eta\},\{\eta\}, \eta)$ is a well of depth $\lambda_{N}(\eta)^{-1}$ in the sense of Definition 2.1.

The first example highlights the role of condition (V3) in preventing some evanescent sets to be called wells.

Example 3.1. Consider the sequence of Markov processes $\left\{\eta_{t}^{N}: t \geq 0\right\}$ on $E=$ $\{-1,0,1\}$ with rates given by

$$
R_{N}(-1,0)=R_{N}(1,0)=N, \quad R_{N}(0,-1)=R_{N}(0,1)=1,
$$

and $R_{N}(j, k)=0$ otherwise. 
Obviously, we do not wish the triple $(\{-1\},\{-1,0\},-1)$ to be a valley. Nevertheless, this triple satisfies conditions (V1) and (V2) of Definition 2.1. The first one is satisfied by default. To check the second one, note that starting from -1

$$
T_{\breve{\mathcal{B}}}=\sum_{j=1}^{M}\left\{S_{j}+T_{j}\right\},
$$

where $\left\{S_{j}: j \geq 1\right\},\left\{T_{j}: j \geq 1\right\}$ are independent sequences of i.i.d. exponential random variables of parameter $N, 1$, respectively, and $M$ is geometric random variable of parameter $1 / 2$, independent of the sequences. Hence, $(1 / 2) T_{\mathcal{B}^{c}}$ converges in distribution, as $N \uparrow \infty$, to a mean 1 exponential random variable.

It is condition (V3) which prevents the triple $(\{-1\},\{-1,0\},-1)$ to be a valley since the time spent at 0 before reaching $\{-1,1\}$ is a mean $1 / 2$ exponential random variable.

Next example illustrates the fact that conditions (V2), (V3) may hold while (V1) fails.

Example 3.2. Consider the Markov process on $\{0,1,2,3\}$ with rates given by

$$
\begin{aligned}
& R_{N}(1,0)=R_{N}(2,3)=1-(1 / N), \quad R_{N}(1,2)=R_{N}(2,1)=1 / N, \\
& \quad R_{N}(0,0)=a, \quad R_{N}(3,3)=b
\end{aligned}
$$

for some $a, b>0$, and $R_{N}(i, j)=0$ otherwise.

Consider the tripe $(\{1,2\},\{1,2\}, 1)$. It is clear that condition (V1) does not hold since the process starting from 2 reaches $\mathcal{B}^{c}=\{0,3\}$ before hitting 1 with probability $1-(1 / N)$. Condition (V2) is fulfilled for $\theta=1$ because $T_{\mathcal{B}^{c}}$ converges to a mean one exponential time, independently from the starting point, and condition (V3) is in force by default.

The third example illustrates the fact that the depth of a valley depends on the basin.

Example 3.3. Consider the sequence of Markov processes $\left\{\eta_{t}^{N}: t \geq 0\right\}$ on $E=$ $\{-1,0,1\}$ with rates given by

$$
R_{N}(-1,0)=R_{N}(1,0)=1, \quad R_{N}(0,-1)=R_{N}(0,1)=N,
$$

and $R_{N}(j, k)=0$ otherwise.

By the observation of the beginning of this section, the triple $(\{-1\},\{-1\},-1)$ is a valley of depth 1 . On the other hand, the triple $(\{-1\},\{-1,0\},-1)$ is a valley of depth 2. Condition (V1) is satisfied by default, and condition (V2) can be verified by representing the time needed to reach $\mathcal{B}^{c}$ as a geometric sum of independent exponential random variables, as in Example 3.1. Requirement (V3) is readily checked.

Next example shows that conditions (V1), (V2) and (2.5) do not imply (V3).

Example 3.4. Consider the sequence of Markov processes $\left\{\eta_{t}^{N}: t \geq 0\right\}$ on $E=$ $\{1,2,3\}$ with rates given by $R_{N}(1,2)=N, R_{N}(2,1)=N-1, R(2,3)=1$ and $R_{N}(i, j)=0$ otherwise. 
Let $\xi=1, W=\{1\}$ and $B=\{1,2\}$. Condition (V1) is fulfilled by default. Condition (V2) is easily checked for $\theta_{N}=2$. In fact, the hitting time $\tau_{3}^{N}$ of 3 starting from 1 can be written as $\sum_{1 \leq j \leq M}\left\{S_{j}+T_{j}\right\}$, where $\left\{S_{j}: j \geq 1\right\},\left\{T_{j}: j \geq 1\right\}$ are independent sequences of i.i.d. mean $1 / N$ exponential random variables and $M$ is a geometric random variable of parameter $1 / N$, independent of both sequences. It follows from this representation that $\tau_{3}^{N} / 2$ converges in distribution to a mean 1 exponential random variable.

For similar reasons, conditions (V3) fails: With the notation just introduced, starting from 1, the time spent at state 2 before hitting 3, denoted in Section 2 by $T_{3}^{N}(2)$, converges to a mean 1 exponential random variable.

Condition (2.5), however, is in force, since the hitting time of the set $\{1,3\}$ starting from 2 is of order $1 / N$.

The fifth example shows that metastates might not be wells of valleys. It presents also a triple which fulfills condition (V1), (V3) but not (V2) nor (V1').

Example 3.5. Consider the sequence of Markov processes $\left\{\eta_{t}^{N}: t \geq 0\right\}$ on $E=$ $\{0,1,2\}$ with rates given by

$$
R_{N}(1,0)=N-1, R_{N}(1,2)=1, R_{N}(2,1)=N^{-1}, R_{N}(0,1)=N^{2},
$$

and $R_{N}(j, k)=0$ otherwise.

The triple $(\{1,2\},\{1,2\}, 2)$ is not a well because condition (V1) is violated. With overwhelming probability the process starting from 1 leaves the set $\{1,2\}$ before reaching 2 . The triple $(\{1,2\},\{1,2\}, 1)$ is not a well either. While conditions (V1), (V3) are clearly satisfied, it is not difficult to show that condition (V2) is violated. In fact, starting from $1, T_{\mathcal{B}^{c}}$ converges to a mean one exponential random variable, while starting from $2, N^{-1} T_{\mathcal{B} c}$ converges to a mean one exponential random variable. It is also clear that condition (V1') fails in this case since on the scale of order 1 the process starting from 2 never reaches 1 .

At the scale $N^{-2}$ the process exhibits a tunneling behaviour, as described in Definition 2.3. with metastates $\mathcal{E}^{1}=\{0\}$ and $\mathcal{E}^{2}=\{1,2\}, \boldsymbol{\xi}_{2}=1$, and asymptotic Markov dynamics characterized by the rates $r(1,2)=1, r(2,1)=0$. It does not exhibit a metastable behaviour, as described in Definition [2.5 because condition (M1') is violated. Starting from state $2 \in \mathcal{E}^{2}$, the process never reaches the attractor 1 in the time scale $N^{-2}$. We have also here an example of an absorbing set for the asymptotic dynamics which is not a valley due to the existence of the well $\{2\}$ in the the set $\mathcal{E}^{2}$ of depth $N \gg N^{-2}$.

Next example shows that there might exist inaccessible valleys.

Example 3.6. Consider the sequence of Markov processes $\left\{\eta_{t}^{N}: t \geq 0\right\}$ on $E=$ $\{1, \ldots, 5\}$ with rates given by

$$
\begin{aligned}
& R_{N}(j, k)=1 \text { if } j \text { is even, } k \text { odd and }|j-k|=1, \\
& R_{N}(1,2)=R_{N}(3,4)=R_{N}(5,4)=N^{-1}, \quad R_{N}(3,2)=N^{-2}, \\
& R_{N}(j, k)=0 \quad \text { otherwise. }
\end{aligned}
$$

The triples $(\{1\},\{1,2\}, 1),(\{3\},\{3,4\}, 3),(\{5\},\{4,5\}, 5)$ are valleys of depth $2 N$. Moreover, at the time scale $N$ the process exhibits a metastable behaviour, as described in Definition 2.5, with metastates $\mathcal{E}^{1}=\{1\}, \mathcal{E}^{2}=\{3\}, \mathcal{E}^{3}=\{5\}$ and asymptotic Markov dynamics characterized by the rates $r(1,2)=r(2,3)=$ 
$r(3,2)=1 / 2, r(i, j)=0$, otherwise. Note that the metastate $\mathcal{E}^{1}$ is inaccessible in the sense that $r(2,1)+r(3,1)=0$. This means that in the time scale $N$ the process starting from 1 eventually leaves this state, never to return.

The penultimate example, very instructive, shows that different phenomena may be observed on different scales. It also highlights the role of conditions (V3), (V3').

Example 3.7. Consider the sequence of Markov processes $\left\{\eta_{t}^{N}: t \geq 0\right\}$ on $E=$ $\{1, \ldots, 5\}$ with rates given by

$$
\begin{aligned}
& R_{N}(j, k)=1 \quad \text { if } j \text { is even, } k \text { odd and }|j-k|=1, \\
& R_{N}(1,2)=N^{-2}, \quad R_{N}(3,2)=N^{-3}, \quad R_{N}(3,4)=R_{N}(5,4)=N^{-1}, \\
& \quad R_{N}(j, k)=0 \quad \text { otherwise. }
\end{aligned}
$$

A simple computation shows that the measure $m_{N}$ on $E$ given by $m_{N}(1)=N^{2}$, $m_{N}(2)=1, m_{N}(3)=N^{3}, m_{N}(4)=N^{2}, m_{N}(5)=N^{3}$ is reversible for the Markov process. We leave to the reader to check that $(\{3\},\{3,4\}, 3),(\{5\},\{4,5\}, 5)$ are valleys of depth $2 N$, and that $(\{1\},\{1,2\}, 1),(\{3,4,5\},\{3,4,5\}, 3),(\{3,4,5\},\{2,3,4$, $5\}, 3)$ are valleys of depth $2 N^{2}, 2 N^{3}, 4 N^{3}$, respectively. The presence of valleys of different depths leads to diverse tunneling behaviors at different time scales.

This example illustrates that we may have valleys satisfying conditions (V1), (V2) and (V3), but not (V3') and (2.5). This is the case of the triple $(\{3\},\{1,2,3$, $4\}, 3)$. The latter conditions are violated because the annulus $\{1,2,4\}$ contains the valley $(\{1\},\{1,2\}, 1)$ of depth $2 N^{2}$, larger than $2 N$ which is the depth of $(\{3\},\{3,4\}, 3)$. On the scale $N$, the process starting from 1 never reaches 3 with positive probability. However, condition (V3) holds because on the scale $N$ the process starting from 3 never reaches $\{1,2\}$.

Note that transferring the points 1,2 from $\Delta$ to $\mathcal{B}^{c}$, we transform the the valley $(\{3\},\{1,2,3,4\}, 3)$ in the S-valley $(\{3\},\{3,4\}, 3)$.

At the scale $N$ one observes a tunneling between $\mathcal{E}^{1}=\{3\}$ and $\mathcal{E}^{2}=\{5\}$, characterized by the asymptotic Markov rates $r(1,2)=r(2,1)=1 / 2$. Assumption (M3') is not satisfied because the set $\Delta_{N}$ contains a well of depth larger than the depth of the metastates. However, this well is never visited if the process starts from one of the metastates.

To turn the tunneling behavior into a metastable one, we may add the metastate $\mathcal{E}^{3}=\{1\}$ and show that at scale $N$, the process exhibits a metastable behaviour with metastates $\mathcal{E}^{1}=\{3\}, \mathcal{E}^{2}=\{5\}, \mathcal{E}^{3}=\{1\}$ and asymptotic Markov dynamics characterized by the rates $r(1,2)=r(2,1)=1 / 2, r(i, j)=0$, otherwise. Observe that an isolated state has appeared in the asymptotic dynamics.

At scale $N^{2}$, the metastates $\mathcal{E}^{1}=\{3\}, \mathcal{E}^{2}=\{5\}$ coalesce into one deeper well. In this scale the process exhibits the metastable behaviour with metastates $\mathcal{E}^{1}=$ $\{1\}, \mathcal{E}^{2}=\{3,4,5\}$, and asymptotic Markov dynamics characterized by the rates $r(1,2)=1 / 2, r(2,1)=0$. Note that we have here an absorbing asymptotic state and that $\{3,4,5\}$ is not the well of a valley of depth of order $N^{2}$, but the well of a valley of depth of order $N^{3}$.

The last example shows that the existence of an attractor is superfluous in the definition of a valley. Denote by $E_{N}=(\mathbb{Z} / N \mathbb{Z})^{d} \cup(\mathbb{Z} / N \mathbb{Z})^{d}$ the union of two $d$ dimensional torii of length $N$ and denote by $(x, j), x \in(\mathbb{Z} / N \mathbb{Z})^{d}, j= \pm 1$, the elements of $E_{N}$. 
Example 3.8. Consider the sequence of Markov processes $\left\{\eta_{t}^{N}: t \geq 0\right\}$ on $E_{N}$ with rate jumps given by

$$
R_{N}\left((x, j),\left(x^{\prime}, j\right)\right)=\frac{1}{2 d} \mathbf{1}\left\{\left|x-x^{\prime}\right|=1\right\}, \quad R_{N}((x, j),(x,-j))=\frac{1}{\theta_{N}},
$$

for some rate $\theta_{N}$ such that $N^{2} \ll \theta_{N} \ll N^{d}$, and $R_{N}((x, i),(y, j))=0$ otherwise.

It is well known that the spectral gap of the symmetric simple random walk on the torus $(\mathbb{Z} / N \mathbb{Z})^{d}$ is of order $N^{-2}$. The evolution of the process $\eta_{t}^{N}$ is therefore quite clear. In a time scale of order $N^{2}$, the process thermalizes in the torus where it started from, and after an exponential time of order $\theta_{N}$ it jumps to the other torus, replicating there the same qualitative behavior.

Hence, each torus satisfies all reasonable conditions to be qualified as a valley of depth $\theta_{N}$. Nevertheless, there is no attractor in this example since a specific state is visited by the symmetric simple random walk only in the scale $N^{d}$.

\section{VAlLEys AND Metastability}

In this section we prove some results on valleys and on tunneling. The first lemma states that we may replace condition (V1) by condition (V1') in the definition of a valley.

Lemma 4.1. In Definition 2.1, condition (V1) may be replaced by condition (V1').

Proof. Let us denote by $\Theta_{t}:=\Theta_{t}^{N}, t \geq 0$, the time-shift operators on the path space $D\left(\mathbb{R}_{+}, E_{N}\right)$. Let $(\mathcal{W}, \mathcal{B}, \boldsymbol{\xi})$ be a valley of depth $\boldsymbol{\theta}=\left(\theta_{N}: N \geq 1\right)$. Fix a point $\boldsymbol{\eta}=\left(\eta_{N}: N \geq 1\right)$ in $\mathcal{W}$ as the starting point. Consider the pair of random variables $T_{\boldsymbol{\xi}}, T_{\mathcal{B}^{c}} \circ \Theta_{T_{\boldsymbol{\xi}}}$, which are independent by the strong Markov property. According to assumption (V1), the event $\left\{T_{\boldsymbol{\xi}}<T_{\mathcal{B}^{c}}\right\}$ has asymptotic probability equal to one. On this event $T_{\boldsymbol{\xi}}+T_{\mathcal{B}^{c}} \circ \Theta_{T_{\xi}}=T_{\mathcal{B}^{c}}$. Since, by assumption (V1), $\theta_{N}^{-1} T_{\mathcal{B}^{c}}$ converges to a mean one exponential random variable, $\theta_{N}^{-1}\left\{T_{\boldsymbol{\xi}}+T_{\mathcal{B}^{c}} \circ \Theta_{T_{\xi}}\right\}$ also converges to a mean one exponential random variable.

Suppose by contradiction that there exist $\delta, \epsilon>0$ such that

$$
\limsup _{N \rightarrow \infty} \mathbf{P}_{\eta_{N}}\left[\frac{1}{\theta_{N}} T_{\boldsymbol{\xi}}>\delta\right]=\epsilon .
$$

By assumptions $(\mathbf{V 1}),(\mathbf{V} 2), \theta_{N}^{-1}\left(T_{\mathcal{B} c} \circ \Theta_{T_{\xi}}\right)$ converges to a mean one exponential random variable and, by (4.1), $\theta_{N}^{-1} T_{\boldsymbol{\xi}}>\delta$ with strictly positive probability. In particular, $\theta_{N}^{-1}\left\{T_{\boldsymbol{\xi}}+T_{\mathcal{B}^{c}} \circ \Theta_{T_{\xi}}\right\}$ may not converge to an exponential random variable, in contradiction with the conclusion reached above.

Conversely, the event $\left\{T_{\boldsymbol{\xi}}<T_{\mathcal{B}^{c}}\right\}$ contains the event $\left\{T_{\boldsymbol{\xi}}<\delta \theta_{N}\right\} \cap\left\{T_{\mathcal{B}^{c}}>\delta \theta_{N}\right\}$ for every $\delta>0$. By assumptions (V1'), (V2), the $\mathbf{P}_{\eta_{N}}$ - probability of this event converges to 1 as $N \uparrow \infty$ and then $\delta \downarrow 0$. This concludes the proof of the lemma.

The second result examines the assumptions (V3) and (V3') in the definition of valleys.

Lemma 4.2. In Definition 2.1, assumption (V3) may be replaced by (2.4), and in Definition 2.2 assumption (V3') may be replaced by (2.6). 
Proof. The time integral in (2.4) is bounded above by $\min \left\{t, \theta_{N}^{-1} T_{\mathcal{B}^{c}}(\boldsymbol{\Delta})\right\}$. Therefore, (2.4) follows from (V3).

Conversely, the time integral in (2.4) is bounded below by $\theta_{N}^{-1} T_{\mathcal{B}^{c}}(\boldsymbol{\Delta}) \mathbf{1}\left\{T_{\mathcal{B}^{c}} \leq\right.$ $\left.t \theta_{N}\right\}$. This expression is itself bounded below by $\min \left\{\theta_{N}^{-1} T_{\mathcal{B}^{c}}(\boldsymbol{\Delta}), a\right\} \mathbf{1}\left\{T_{\mathcal{B}^{c}} \leq t \theta_{N}\right\}$ for every $a>0$. Therefore,

$$
\min \left\{\theta_{N}^{-1} T_{\mathcal{B}^{c}}(\boldsymbol{\Delta}), a\right\} \leq \int_{0}^{\min \left\{t, \theta_{N}^{-1} T_{\mathcal{B}^{c}}\right\}} \mathbf{1}\left\{\eta_{s \theta_{N}} \in \Delta_{N}\right\} d s+a \mathbf{1}\left\{T_{\mathcal{B}^{c}}>t \theta_{N}\right\}
$$

for every $a>0$. Fix a point $\boldsymbol{\eta}=\left(\eta^{N}: N \geq 1\right)$ in $\mathcal{W}$. By (2.4), the expectation with respect to $\mathbf{P}_{\eta^{N}}$ of the first term on the right hand side vanishes as $N \uparrow \infty$ for every $t>0$. By (V2), the expectation with respect to $\mathbf{P}_{\eta^{N}}$ of the second term vanishes as $N \uparrow \infty$ and then $t \uparrow \infty$. Therefore, for every $a>0$

$$
\lim _{N \rightarrow \infty} \mathbf{E}_{\eta^{N}}\left[\min \left\{\theta_{N}^{-1} T_{\mathcal{B}^{c}}(\boldsymbol{\Delta}), a\right\}\right]=0
$$

This proves (V3).

In the same way we prove that we may substitute assumption (V3') by (2.6) in Definition 2.2. This concludes the proof of the lemma.

Next lemma is needed in the proof of Proposition 4.4. one of the main results of this section.

Lemma 4.3. Consider a subset $\mathcal{A}=\left(A_{N}: N \geq 1\right)$ of $\left(E_{N}: N \geq 1\right)$. Assume that there exists $t>0$ and $\epsilon<1$ such that

$$
\limsup _{N \rightarrow \infty} \sup _{\eta \in W_{N}} \mathbf{P}_{\eta}\left[T_{\mathcal{A}}>t \theta_{N}\right]<\epsilon .
$$

Then, $\sup _{\eta \in W_{N}} \mathbf{E}_{\eta}\left[T_{\mathcal{A}}(\mathcal{W})\right] \leq[t /(1-\epsilon)] \theta_{N}$ for every $N$ sufficiently large and

$$
\lim _{K \rightarrow \infty} \limsup _{N \rightarrow \infty} \sup _{\eta \in W_{N}} \mathbf{E}_{\eta}\left[\theta_{N}^{-1} T_{\mathcal{A}}(\mathcal{W}) \mathbf{1}\left\{T_{\mathcal{A}}(\mathcal{W})>K \theta_{N}\right\}\right]=0 .
$$

Proof. The proof is a simple consequence of the strong Markov property and assumption (4.2). Consider the sequence of stopping times $\left\{I_{k}: k \geq 1\right\},\left\{J_{k}: k \geq 1\right\}$ defined as follows. $I_{1}=0, J_{1}=t \theta_{N}$,

$$
I_{k+1}=\inf \left\{t>J_{k}: \eta_{t}^{N} \in W_{N}\right\}, \quad J_{k+1}=I_{k+1}+t \theta_{N}, \quad k \geq 1,
$$

with the convention that $J_{k}=I_{k+1}=\infty$ if $I_{k}=\infty$ for some $k \geq 1$. Let $M$ be the first time interval $\left[I_{k}, J_{k}\right]$ in which the process visits $A_{N}$ :

$$
M=\min \left\{k \geq 1: \eta_{t}^{N} \in A_{N} \text { for some } t \in\left[I_{k}, J_{k}\right] \text { or } I_{k}=\infty\right\} .
$$

Clearly, $T_{\mathcal{A}}(\mathcal{W}) \leq t \theta_{N} M$. On the other hand, for $N$ sufficiently large, by definition of the stopping times $\left\{I_{k}: k \geq 1\right\}$ and by assumption (4.2), $M$ is stochastically dominated by a random variable $M^{\prime}$ with geometric distribution given by $P\left[M^{\prime}=\right.$ $k]=(1-\epsilon) \epsilon^{k-1}, k \geq 1$. This concludes the proof of the lemma.

Next proposition gives an equivalent definition of a valley with attractor.

Proposition 4.4. Assume that $(\mathcal{W}, \mathcal{B}, \boldsymbol{\xi})$ is a valley of depth $\boldsymbol{\theta}$ and attractor $\boldsymbol{\xi}$. Then, for any point $\boldsymbol{\eta}=\left(\eta^{N}: N \geq 1\right)$ in $\mathcal{W}$, 
(i) The hitting time of the attractor $\boldsymbol{\xi}$ is negligible with respect to the escape time from the basin $\mathcal{B}$ in the sense that

$$
\lim _{N \rightarrow \infty} \frac{\mathbf{E}_{\eta^{N}}\left[T_{\boldsymbol{\xi}}(\mathcal{W})\right]}{\mathbf{E}_{\eta^{N}}\left[T_{\mathcal{B}^{c}}(\mathcal{W})\right]}=0
$$

(ii) Under $\mathbf{P}_{\eta^{N}}$, the law of the random variable $T_{\mathcal{B}^{c}}(\mathcal{W}) / \mathbf{E}_{\eta^{N}}\left[T_{\mathcal{B}^{c}}(\mathcal{W})\right]$ converges to a mean-one exponential distribution;

(iii) For every $\delta>0$,

$$
\lim _{N \rightarrow \infty} \mathbf{P}_{\eta^{N}}\left[\frac{T_{\mathcal{B}^{c}}(\boldsymbol{\Delta})}{\mathbf{E}_{\eta^{N}}\left[T_{\mathcal{B}^{c}}(\mathcal{W})\right]}>\delta\right]=0 .
$$

Moreover, the sequences $\theta_{N}$ and $\mathbf{E}_{\eta^{N}}\left[T_{\mathcal{B}^{c}}(\mathcal{W})\right]$ are asymptotically equivalent in the sense that $\lim _{N \rightarrow \infty} \theta_{N}^{-1} \mathbf{E}_{\eta^{N}}\left[T_{\mathcal{B}^{c}}(\mathcal{W})\right]=1$,

Conversely, if $(\mathcal{W}, \mathcal{B}, \boldsymbol{\xi})$ is a triple satisfying (2.3) for which (i) - (iii) hold, then for any point $\boldsymbol{\eta}=\left(\eta^{N}: N \geq 1\right)$ in $\mathcal{W}$, the sequence $\mathbf{E}_{\eta^{N}}\left[T_{\mathcal{B}^{c}}(\mathcal{W})\right]$ is asymptotically equivalent to $\mathbf{E}_{\xi^{N}}\left[T_{\mathcal{B}^{c}}(\mathcal{W})\right]$ :

$$
\lim _{N \rightarrow \infty} \frac{\mathbf{E}_{\eta^{N}}\left[T_{\mathcal{B}^{c}}(\mathcal{W})\right]}{\mathbf{E}_{\xi^{N}}\left[T_{\mathcal{B}^{c}}(\mathcal{W})\right]}=1
$$

and $(\mathcal{W}, \mathcal{B}, \boldsymbol{\xi})$ is a valley of depth $\boldsymbol{\theta}$, where $\theta_{N}=\mathbf{E}_{\xi^{N}}\left[T_{\mathcal{B}^{c}}(\mathcal{W})\right]$.

It is implicit in the statement of this proposition that the time spent in the well $\mathcal{W}$ before leaving the basin $\mathcal{B}, T_{\mathcal{B}^{c}}(\mathcal{W})$, has finite expectation with respect to any $\mathbf{P}_{\eta^{N}}$ for sufficiently large $N$, as well as the time spent in the well $\mathcal{W}$ before reaching the attractor $\boldsymbol{\xi}, T_{\boldsymbol{\xi}}(\mathcal{W})$.

Proof of Proposition 4.4. Assume that $(\mathcal{W}, \mathcal{B}, \boldsymbol{\xi})$ is a valley of depth $\boldsymbol{\theta}$ and attractor $\xi$. We first claim that

$$
\lim _{N \rightarrow \infty} \sup _{\eta \in W_{N}} \mathbf{E}_{\eta}\left[\theta_{N}^{-1} T_{\boldsymbol{\xi}}(\mathcal{W})\right]=0 .
$$

This assertion follows from (V1') and the previous lemma with $\mathcal{A}=\{\boldsymbol{\xi}\}, t=\delta$, $\epsilon=1 / 2$.

Fix a point $\boldsymbol{\eta}=\left(\eta^{N}: N \geq 1\right)$ in $\mathcal{W}$. We claim that

$$
\lim _{N \rightarrow \infty} \mathbf{E}_{\eta^{N}}\left[\theta_{N}^{-1} T_{\mathcal{B}^{c}}(\mathcal{W})\right]=1
$$

Three ingredients are needed to prove this result. The convergence of $\theta_{N}^{-1} T_{\mathcal{B}^{c}}$ to a mean one exponential random variable, a bound on $\mathbf{E}_{\eta^{N}}\left[\theta_{N}^{-1} T_{\mathcal{B}^{c}}(\mathcal{W})\right]$ provided by the previous lemma, and the fact that the process does not spend too much time in $\Delta$.

We start with the proof of the lower bound. Fix $\delta>0, t>0$. On the set $\left\{T_{\mathcal{B}^{c}}(\boldsymbol{\Delta}) \leq \delta \theta_{N}\right\}$, we have that $T_{\mathcal{B}^{c}}(\mathcal{W}) \geq T_{\mathcal{B}^{c}}-\delta \theta_{N}$. Therefore,

$$
T_{\mathcal{B}^{c}}(\mathcal{W}) \geq-\delta \theta_{N}+T_{\mathcal{B}^{c}} \mathbf{1}\left\{T_{\mathcal{B}^{c}}(\boldsymbol{\Delta}) \leq \delta \theta_{N}\right\} .
$$

Replacing $T_{\mathcal{B}^{c}}$ by $\min \left\{T_{\mathcal{B}^{c}}, t \theta_{N}\right\}$ we obtain the estimate

$$
T_{\mathcal{B}^{c}}(\mathcal{W}) \geq-\delta \theta_{N}-t \theta_{N} \mathbf{1}\left\{T_{\mathcal{B}^{c}}(\boldsymbol{\Delta})>\delta \theta_{N}\right\}+\min \left\{T_{\mathcal{B}^{c}}, t \theta_{N}\right\}
$$

which holds for all $\delta>0, t>0$.

By (V3), the expectation with respect to $\mathbf{P}_{\eta^{N}}$ of the second term on the right hand side divided by $\theta_{N}$ vanishes as $N \uparrow \infty$ for any fixed $\delta>0, t>0$. By (V2), the 
expectation with respect to $\mathbf{P}_{\eta^{N}}$ of the third term on the right hand side divided by $\theta_{N}$ converges to 1 as $N \uparrow \infty$ and then $t \uparrow \infty$. Therefore,

$$
\liminf _{N \rightarrow \infty} \mathbf{E}_{\eta^{N}}\left[\theta_{N}^{-1} T_{\mathcal{B}^{c}}(\mathcal{W})\right] \geq 1 .
$$

The proof of the upper bound is simpler. For every $A>0$,

$$
\mathbf{E}_{\eta^{N}}\left[T_{\mathcal{B}^{c}}(\mathcal{W})\right] \leq \mathbf{E}_{\eta^{N}}\left[\min \left\{T_{\mathcal{B}^{c}}, A \theta_{N}\right\}\right]+\mathbf{E}_{\eta^{N}}\left[T_{\mathcal{B}^{c}}(\mathcal{W}) \mathbf{1}\left\{T_{\mathcal{B}^{c}}(\mathcal{W})>A \theta_{N}\right\}\right]
$$

By (V2), the first term on the right hand side divided by $\theta_{N}$ converges to 1 as $N \uparrow \infty$ and then $A \uparrow \infty$. By (V2), (4.2) holds with $\mathcal{A}=\mathcal{B}^{c}, \epsilon=1 / 2$ and some $t<\infty$. Therefore, by (4.3), the second term divided by $\theta_{N}$ vanishes as $N \uparrow \infty$ and then $A \uparrow \infty$. This concludes the proof of (4.5).

Assertion (i) follows from (4.4) and (4.5), and assertion (iii) from (V3) and (4.5). Finally, $T_{\mathcal{B}^{c}}(\mathcal{W})=T_{\mathcal{B}^{c}}-T_{\mathcal{B}^{c}}(\boldsymbol{\Delta})$. By $(\mathbf{V} 2), \theta_{N}^{-1} T_{\mathcal{B}^{c}}$ converges in distribution to a mean one exponential random variable, and, by $(\mathbf{V 3}), \theta_{N}^{-1} T_{\mathcal{B}^{c}}(\boldsymbol{\Delta})$ converges to 0 in probability. Assertion (ii) follows from these facts and from (4.5). The final claim of the first part of the proposition has been proved in (4.4).

To prove the converse, suppose that conditions (i) - (iii) hold. We first prove that (V1), (V2), (V3) are in force with $\theta_{N}$ replaced by the sequence $\theta\left(\eta^{N}\right)=$ $\mathbf{E}_{\eta^{N}}\left[T_{\mathcal{B}^{c}}(\mathcal{W})\right]$, which depends on the point $\boldsymbol{\eta}=\left(\eta^{N}: N \geq 1\right)$. In this case, condition (V3) corresponds to (iii). To prove (V2), note that $T_{\mathcal{B}^{c}}=T_{\mathcal{B}^{c}}(\mathcal{W})+T_{\mathcal{B}^{c}}(\boldsymbol{\Delta})$. By (ii), $\theta\left(\eta^{N}\right)^{-1} T_{\mathcal{B}^{c}}(\mathcal{W})$ converges in distribution to a mean one exponential random variable and, by (iii), $\theta\left(\eta^{N}\right)^{-1} T_{\mathcal{B}^{c}}(\boldsymbol{\Delta})$ vanishes in probability. Therefore, (V2) holds. Finally, on the set $\left\{T_{\boldsymbol{\xi}}<T_{\mathcal{B}^{c}}\right\}, T_{\boldsymbol{\xi}}=T_{\boldsymbol{\xi}}(\mathcal{W})+T_{\boldsymbol{\xi}}(\boldsymbol{\Delta})$ and $T_{\boldsymbol{\xi}}(\boldsymbol{\Delta}) \leq T_{\mathcal{B}^{c}}(\boldsymbol{\Delta})$. By (i) and (iii), $\theta\left(\eta^{N}\right)^{-1} T_{\boldsymbol{\xi}}(\mathcal{W})$ and $\theta\left(\eta^{N}\right)^{-1} T_{\mathcal{B}^{c}}(\boldsymbol{\Delta})$ vanish in probability as $N \uparrow \infty$. On the other hand, by (V2), already proved, $\theta\left(\eta^{N}\right)^{-1} T_{\mathcal{B}^{c}}$ converges in distribution to a mean one exponential variable. This proves $(\mathbf{V} \mathbf{1})$.

It remains to show that the sequences $\theta\left(\eta^{N}\right)=\mathbf{E}_{\eta^{N}}\left[T_{\mathcal{B}^{c}}(\mathcal{W})\right]$ and $\mathbf{E}_{\xi^{N}}\left[T_{\mathcal{B}^{c}}(\mathcal{W})\right]$ are asymptotically equivalent in the sense that their ratio converges to 1.

By (ii) and Lemma 4.3, the sequence $\theta\left(\eta^{N}\right)^{-1} T_{\mathcal{B}^{c}}(\mathcal{W})$ is uniformly integrable with respect to $\mathbf{P}_{\eta^{N}}$. Therefore, by $(\mathbf{V 1})$,

$$
\lim _{N \rightarrow \infty} \frac{1}{\theta\left(\eta^{N}\right)} \mathbf{E}_{\eta^{N}}\left[T_{\mathcal{B}^{c}}(\mathcal{W}) \mathbf{1}\left\{T_{\boldsymbol{\xi}}<T_{\mathcal{B}^{c}}\right\}\right]=1 .
$$

By the strong Markov property and the explicit form of $T_{\mathcal{B}^{c}}(\mathcal{W})$, the expectation is equal to

$$
\frac{1}{\theta\left(\eta^{N}\right)} \mathbf{E}_{\eta^{N}}\left[T_{\boldsymbol{\xi}}(\mathcal{W}) \mathbf{1}\left\{T_{\boldsymbol{\xi}}<T_{\mathcal{B}^{c}}\right\}\right]+\frac{1}{\theta\left(\eta^{N}\right)} \mathbf{E}_{\xi^{N}}\left[T_{\mathcal{B}^{c}}(\mathcal{W})\right] \mathbf{P}_{\eta^{N}}\left[T_{\boldsymbol{\xi}}<T_{\mathcal{B}^{c}}\right] .
$$

By (i), the first term vanishes as $N \uparrow \infty$. Since by (V1) $\mathbf{P}_{\eta^{N}}\left[T_{\boldsymbol{\xi}}<T_{\mathcal{B}^{c}}\right]$ converges to $1, \mathbf{E}_{\eta^{N}}\left[T_{\mathcal{B}^{c}}(\mathcal{W})\right]$ and $\mathbf{E}_{\xi^{N}}\left[T_{\mathcal{B}^{c}}(\mathcal{W})\right]$ are asymptotically equivalent. This concludes the proof of the proposition.

We conclude this section with the proofs of Propositions 2.4 and 2.6. Let us first fix a metric in the path space $D\left(\mathbb{R}_{+}, S \cup\{\mathfrak{d}\}\right)$ which induces the Skorohod topology. In what follows, we identify the point $\mathfrak{d}$ with $0 \in \mathbb{Z}$ so that $S \cup\{\mathfrak{d}\}$ is a metric space with the metric induced by $\mathbb{Z}$.

For each integer $m \geq 1$, let $\Lambda_{m}$ denote the class of strictly increasing, continuous mappings of $[0, m]$ onto itself. If $\lambda \in \Lambda_{m}$, then $\lambda_{0}=0$ and $\lambda_{m}=m$. In addition, 
consider the function

$$
g_{m}(t)= \begin{cases}1 & \text { if } t \leq m-1, \\ m-t & \text { if } m-1 \leq t \leq m, \\ 0 & \text { if } t \geq m .\end{cases}
$$

For any integer $m \geq 1$ and $e, \hat{e} \in D\left(\mathbb{R}_{+}, S \cup\{\mathfrak{d}\}\right)$, define $d_{m}(e, \hat{e})$ to be the infimum of those positive $\epsilon$ for which there exists in $\Lambda_{m}$ a $\lambda$ satisfying

$$
\sup _{t \in[0, m]}\left|\lambda_{t}-t\right|<\epsilon
$$

and

$$
\sup _{t \in[0, m]}\left|g_{m}\left(\lambda_{t}\right) e_{\lambda_{t}}-g_{m}(t) \hat{e}_{t}\right|<\epsilon .
$$

Finally, we define the metric in $D\left(\mathbb{R}_{+}, S \cup\{\mathfrak{d}\}\right)$ by

$$
d(e, \hat{e})=\sum_{m=1}^{\infty} 2^{-m}\left(1 \wedge d_{m}(e, \hat{e})\right) .
$$

This metric induces the Skorohod topology in the path space $D\left(\mathbb{R}_{+}, S \cup\{\mathfrak{d}\}\right.$ ) (cf. [2]).

For any path $e \in D\left(\mathbb{R}_{+}, S \cup\{\mathfrak{d}\}\right)$ denote by $\left(\tau_{n}(e): n \geq 0\right)$ the sequence of jumping times of $e$ : Set $\tau_{0}(e)=0$ and, for $n \geq 1$, we define $\tau_{n}(e)$ as

$$
\tau_{n}(e):=\inf \left\{t>\tau_{n-1}(e): e_{t} \neq e_{\tau_{n-1}(e)}\right\},
$$

with the convention that $\tau_{n}=\infty$ if $\tau_{n-1}=\infty$ and, as usual, inf $\varnothing=+\infty$.

Proposition 2.6 is a consequence of the following result.

Proposition 4.5. Suppose that $\left\{\eta_{t}^{N}: t \geq 0\right\}, N \geq 1$, satisfies (M3) for any $x \in S$. Then, for any $x \in S$ and point $\boldsymbol{\eta}=\left(\eta^{N}: N \geq 1\right)$ in $\mathcal{E}^{x}$,

$$
\lim _{N \rightarrow \infty} \mathbf{E}_{\eta^{N}}\left[d\left(X^{N}, \hat{X}^{N}\right)\right]=0 .
$$

Proof. Fix arbitrary integers $m \geq 1$ and $N \geq 1$. To keep notation simple, set $\tau_{n}:=\tau_{n}\left(X^{N}\right)$ and $\hat{\tau}_{n}:=\tau_{n}\left(\hat{X}^{N}\right), n \geq 0$. Define the random variables

$$
\mathfrak{n}:=\sup \left\{j \geq 0: \hat{\tau}_{j}<m\right\}
$$

and

$$
T\left(X^{N}\right):=\tau_{\mathfrak{n}+1} \wedge m .
$$

In Lemma 4.6 below we show that $\mathbf{P}_{\eta^{N} \text {-a.s., }}$

$$
d_{m}\left(X^{N}, \hat{X}^{N}\right) \leq|S| \max \left\{\hat{\tau}_{\mathfrak{n}}-\tau_{\mathfrak{n}} ; m-T\left(X^{N}\right)\right\} .
$$

To estimate the right hand side in (4.6), observe that

$$
\hat{\tau}_{\mathfrak{n}}-\tau_{\mathfrak{n}}=\mathcal{T}_{\hat{\tau}_{\mathfrak{n}}}^{\Delta_{N}} \leq \mathcal{T}_{m}^{\Delta_{N}}
$$

where $\mathcal{T}_{t}^{\Delta_{N}}$ is the time spent by $\left\{\eta_{t}^{N}: t \geq 0\right\}$ in $\Delta_{N}$ in the time interval $[0, t]$, introduced in (2.2). On the other hand, in the case $\tau_{\mathfrak{n}+1}<m, m-T\left(X^{N}\right)$ can be written as $m-\left\{\hat{\tau}_{\mathfrak{n}}+\left[\tau_{\mathfrak{n}+1}-\tau_{\mathfrak{n}}\right]\right\}+\left[\hat{\tau}_{\mathfrak{n}}-\tau_{\mathfrak{n}}\right]$. Since $\hat{\tau}_{\mathfrak{n}}-\tau_{\mathfrak{n}}$ is the time spent by $\left\{\eta_{t}^{N}: t \geq 0\right\}$ in $\Delta_{N}$ in the time interval $\left[0, \hat{\tau}_{\mathfrak{n}}\right]$ and $m-\left\{\hat{\tau}_{\mathfrak{n}}+\left[\tau_{\mathfrak{n}+1}-\tau_{\mathfrak{n}}\right]\right\}$ is the time spent in $\Delta_{N}$ in the time interval $\left[\hat{\tau}_{\mathfrak{n}}, m\right]$,

$$
m-T\left(X^{N}\right) \leq \mathcal{T}_{m}^{\Delta_{N}} .
$$


Therefore, by (4.6) we have just shown that

$$
d\left(X^{N}, \hat{X}^{N}\right) \leq \sum_{m=1}^{\infty} 2^{-m}\left(1 \wedge|S| \mathcal{T}_{m}^{\Delta_{N}}\right)
$$

The desired result follows from this estimate and property (M3).

Lemma 4.6. For any integers $N, m \geq 1$, 4.6) holds $\mathbf{P}_{\eta^{N}}$-almost surely.

Proof. Fix two integers $N, m \geq 1$. All assertions in what follows must be under-

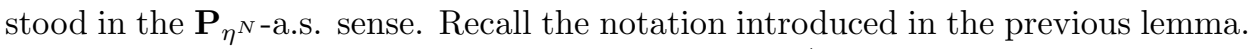

Let us list some evident properties of $X^{N}$ and $\hat{X}^{N}$ : First notice that for all $0 \leq j \leq \mathfrak{n}-1$, we have $\hat{\tau}_{j+1}-\hat{\tau}_{j} \geq \tau_{j+1}-\tau_{j}$ and $X_{s}^{N}=\hat{X}_{t}^{N}$ for $(s, t) \in$ $\left[\tau_{j}, \tau_{j+1}\left[\times\left[\hat{\tau}_{j}, \hat{\tau}_{j+1}\left[\right.\right.\right.\right.$. Furthermore, $\tau_{\mathfrak{n}}<T\left(X^{N}\right) \leq m, X_{\tau_{\mathfrak{n}}}^{N} \neq \mathfrak{d}$ and $X_{s}^{N}=\hat{X}_{t}^{N}$ for $(s, t) \in\left[\tau_{\mathfrak{n}}, T\left(X^{N}\right)\left[\times\left[\hat{\tau}_{\mathfrak{n}}, m[\right.\right.\right.$.

In particular, since $\hat{\tau}_{\mathfrak{n}}<m$, we may choose $\epsilon>0$ small enough such that $\tau_{\mathfrak{n}}<T\left(X^{N}\right)-\epsilon$ and $\hat{\tau}_{\mathfrak{n}}<m-\epsilon$. Now, let $\lambda \in \Lambda_{m}$ be given by: $\lambda_{\hat{\tau}_{j}}=\tau_{j}$, for $j \leq \mathfrak{n}$, $\lambda_{m-\epsilon}=T\left(X^{N}\right)-\epsilon, \lambda_{m}=m$ and we complete $\lambda$ on $[0, m]$ by linear interpolation. Then,

$$
\sup _{t \in[0, m]}\left|\lambda_{t}-t\right| \leq \max \left\{\hat{\tau}_{\mathfrak{n}}-\tau_{\mathfrak{n}}, m-T\left(X^{N}\right)\right\} .
$$

Moreover, since $\lambda_{t} \leq t, 0 \leq t \leq m$,

$$
\begin{aligned}
\sup _{t \in[0, m-\epsilon]}\left|g_{m}\left(\lambda_{t}\right) X_{\lambda_{t}}^{N}-g_{m}(t) \hat{X}_{t}^{N}\right| & \leq|S| \sup _{t \in[0, m-\epsilon]}\left|g_{m}\left(\lambda_{t}\right)-g_{m}(t)\right| \\
& \leq|S| \sup _{t \in[m-1, m-\epsilon]}\left|\lambda_{t}-t\right|
\end{aligned}
$$

and

$$
\begin{aligned}
\sup _{t \in[m-\epsilon, m]}\left|g_{m}\left(\lambda_{t}\right) X_{\lambda_{t}}^{N}-g_{m}(t) \hat{X}_{t}^{N}\right| & \leq|S| \sup _{t \in[m-\epsilon, m]}\left(\left|g_{m}\left(\lambda_{t}\right)-g_{m}(t)\right|+2\left|g_{m}(t)\right|\right) \\
& \leq|S| \sup _{t \in[m-\epsilon, m]}\left|\lambda_{t}-t\right|+2 \kappa \epsilon .
\end{aligned}
$$

Since $\epsilon$ may be taken arbitrary small, the claim is proved.

We now turn to the poof of Proposition 2.4. For every $e \in D\left(\mathbb{R}_{+}, S \cup\{\mathfrak{d}\}\right)$, denote by $J_{t}(e)$ the number of jumps up to time $t$ :

$$
J_{t}(e):=\sup \left\{j \geq 0: \tau_{j}(e) \leq t\right\} .
$$

Proof of Proposition 2.4. Fix an arbitrary non-absorbing state $x_{*} \in S$ for the Markov process $\left\{\mathbb{P}_{x}: x \in S\right\}$, a point $\boldsymbol{\eta}=\left(\eta^{N}: N \geq 1\right)$ in $\mathcal{E}^{x_{*}}$ and a time $t>0$. It suffices to show that $\mathbf{E}_{\eta^{N}}\left[\mathcal{T}_{t}^{\Delta_{N}}\right] \rightarrow 0$ as $N \rightarrow \infty$.

For any integer $K \geq 1$,

$$
\mathcal{T}_{t}^{\Delta_{N}} \leq \mathbf{1}\left\{J_{t}\left(\hat{X}^{N}\right) \geq K\right\} t+\mathbf{1}\left\{J_{t}\left(\hat{X}^{N}\right)<K\right\} \mathcal{T}_{t}^{\Delta_{N}},
$$

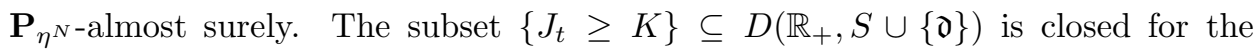
Skorohod topology. Therefore, by property (M2),

$$
\limsup _{N \rightarrow \infty} \mathbf{P}_{\eta^{N}}\left[J_{t}\left(\hat{X}^{N}\right) \geq K\right] \leq \limsup _{N \rightarrow \infty} \mathbf{P}_{\eta^{N}}\left[J_{t}\left(X^{N}\right) \geq K\right] \leq \mathbb{P}_{x^{*}}\left[J_{t} \geq K\right] .
$$

The right hand side vanishes as $K \uparrow \infty$. From this and (4.7), it follows that

$$
\limsup _{N \rightarrow \infty} \mathbf{E}_{\eta^{N}}\left[\mathcal{T}_{t}^{\Delta_{N}}\right] \leq \limsup _{K \uparrow \infty} \limsup _{N \rightarrow \infty} \mathbf{E}_{\eta^{N}}\left[\mathbf{1}\left\{J_{t}\left(\hat{X}^{N}\right)<K\right\} \mathcal{T}_{t}^{\Delta_{N}}\right]
$$


In consequence, in order to conclude the proof it is enough to show that

$$
\lim _{N \rightarrow \infty} \mathbf{E}_{\eta^{N}}\left[\mathbf{1}\left\{J_{t}\left(\hat{X}^{N}\right)=i\right\} \mathcal{T}_{t}^{\Delta_{N}}\right]=0, \quad \forall i \geq 0 .
$$

Fix some integer $i \geq 0$. To keep notation simple, denote $\hat{J}_{t}:=J_{t}\left(\hat{X}^{N}\right)$ and let $\left(\hat{\tau}_{n}: n \geq 0\right)$ stand for the jumping times of $\hat{X}^{N}$. Recall that we denote by $S_{*}$ the set of non-absorbing states for $\left\{\mathbb{P}_{x}: x \in S\right\}$ and set $\mathcal{E}_{N}^{*}=\cup_{x \in S_{*}} \mathcal{E}_{N}^{x}$. On the event $\left\{\hat{J}_{t}=i\right\}$ let us define

$$
I:=\inf \left\{0 \leq j \leq i: \hat{X}_{\hat{\tau}_{j}}^{N} \in S \backslash S_{*}\right\},
$$

so that $I=\infty$ if and only if $\hat{X}_{s} \in S_{*}$, for all $0 \leq s \leq t$. On the one hand, $\mathbf{P}_{\eta^{N}}-$ a.s.,

$$
\begin{aligned}
\mathbf{1}\left\{\hat{J}_{t}=i ; I=\infty\right\} \mathcal{T}_{t}^{\Delta_{N}} & \leq \mathbf{1}\left\{\hat{J}_{t}=i ; I=\infty\right\} \sum_{j=1}^{i+1} \int_{\hat{\tau}_{j-1}}^{\hat{\tau}_{j} \wedge t} \mathbf{1}\left\{\eta_{s}^{N} \in \Delta_{N}\right\} d s \\
& \leq \sum_{j=1}^{i+1} \mathbf{1}\left\{\eta_{\hat{\tau}_{j-1}}^{N} \in \mathcal{E}_{N}^{*}\right\} \int_{\hat{\tau}_{j-1}}^{\hat{\tau}_{j} \wedge t} \mathbf{1}\left\{\eta_{s}^{N} \in \Delta_{N}\right\} d s
\end{aligned}
$$

Thus, applying the strong Markov property we get

$$
\mathbf{E}_{\eta^{N}}\left[\mathbf{1}\left\{\hat{J}_{t}=i ; I=\infty\right\} \mathcal{T}_{t}^{\Delta_{N}}\right] \leq(i+1) \sup _{x \in S_{*}} \sup _{\eta \in \mathcal{E}_{N}^{x}} \mathbf{E}_{\eta}^{N}\left[t \wedge T_{\breve{\mathcal{E}}^{x}}(\boldsymbol{\Delta})\right] .
$$

The right hand side vanishes as $N \rightarrow 0$ by assumption (C1) for the non-absorbing states. On the other hand, for any $0 \leq \ell \leq i$ we have that, $\mathbf{P}_{\eta^{N}}-$ a.s., on the event $\left\{\hat{J}_{t}=i ; I=\ell\right\}$,

$$
\mathcal{T}_{t}^{\Delta_{N}} \leq \sum_{j=1}^{\ell} \int_{\hat{\tau}_{j-1}}^{\hat{\tau}_{j} \wedge t} \mathbf{1}\left\{\eta_{s}^{N} \in \Delta_{N}\right\} d s+\int_{\hat{\tau}_{\ell}}^{\hat{\tau}_{\ell}+t} \mathbf{1}\left\{\eta_{s}^{N} \in \Delta_{N}\right\} d s .
$$

By applying the strong Markov processes as before, we show that $\mathbf{E}_{\eta^{N}}\left[\mathbf{1}\left\{\hat{J}_{t}=\right.\right.$ $\left.i ; I=\ell\} \mathcal{T}_{t}^{\Delta_{N}}\right]$ is bounded above by

$$
\ell \sup _{x \in S_{*}} \sup _{\eta \in \mathcal{E}_{N}^{x}} \mathbf{E}_{\eta}^{N}\left[t \wedge T_{\breve{\mathcal{E}}^{x}}(\boldsymbol{\Delta})\right]+\sup _{\eta \in S \backslash S_{*}} \mathbf{E}_{\eta}^{N}\left[\mathcal{T}_{t}^{\boldsymbol{\Delta}}\right] .
$$

As $N \uparrow \infty$, the first term vanishes as before while the second one vanishes by assumption (M3) for absorbing states. This concludes the proof.

The same proof yields the following version of Proposition 2.4 which does not distinguish between absorbing and non-absorbing states.

Lemma 4.7. Assume that (M2) is fulfilled for a sequence of Markov processes $\left\{\eta_{t}^{N}: t \geq 0\right\}, N \geq 1$. Then, condition (M3) is satisfied if for each $x$ in $S$,

$$
\lim _{N \rightarrow \infty} \sup _{\eta \in \mathcal{E}_{N}^{x}} \mathbf{P}_{\eta}\left[\frac{1}{\theta_{N}} T_{\breve{\varepsilon}^{x}}(\boldsymbol{\Delta})>\delta\right]=0 .
$$

Proof. The proof is simpler than the previous one. We do not need to introduce the variable $I$. We estimate $\mathcal{T}_{t}^{\Delta_{N}}$ as in the case $I=\infty$ to get that

$$
\mathbf{E}_{\eta^{N}}\left[\mathbf{1}\left\{\hat{J}_{t}=i\right\} \mathcal{T}_{t}^{\Delta_{N}}\right] \leq(i+1) \sup _{x \in S} \sup _{\eta \in \mathcal{E}_{N}^{x}} \mathbf{E}_{\eta}^{N}\left[t \wedge T_{\breve{\varepsilon}^{x}}(\boldsymbol{\Delta})\right] .
$$

This expression vanishes as $N \uparrow \infty$ by assumption. 


\section{Proof of the MAIN THEOREMS}

We prove in this section the main results of the article. The proofs rely on some results on recurrent Markov processes presented in Section 6 ,

Proof of Theorem 2.7. Next statement plays a central role in the proof of Theorem 2.7 .

Proposition 5.1. Consider two sequences of sets $\mathcal{W}$ and $\mathcal{B}$ satisfying (2.3). Assume that there exists a point $\boldsymbol{\xi}=\left(\xi^{N}: N \geq 1\right)$ in $\mathcal{W}$ such that for every point $\boldsymbol{\eta}=\left(\eta^{N}: N \geq 1\right)$ in $\mathcal{W}(2.9)$ and (2.10) hold. Then, condition (V1) is in force. Moreover, the law of $r_{N}\left(\mathcal{W}, \mathcal{B}^{c}\right) T_{\mathcal{B}^{c}}(\mathcal{W})$ under $\mathbf{P}_{\eta^{N}}$ converges to a mean-one exponential distribution, as $N \uparrow \infty$, and

$$
\lim _{N \rightarrow \infty} r_{N}\left(\mathcal{W}, \mathcal{B}^{c}\right) \mathbf{E}_{\eta^{N}}\left[T_{\mathcal{B}^{c}}(\mathcal{W})\right]=1
$$

for any point $\boldsymbol{\eta}=\left(\eta^{N}: N \geq 1\right)$ in $\mathcal{W}$

The proof of this proposition is divided in several lemmas. Recall that $\mathcal{E}_{N}=$ $W_{N} \cup B_{N}^{c}, N \geq 1$, and that $\left\{\eta_{t}^{\mathcal{E}_{N}}: t \geq 0\right\}$ stands for the trace of $\left\{\eta_{t}^{N}: t \geq 0\right\}$ on $\mathcal{E}_{N}$. For any $\boldsymbol{\theta}=\left(\theta_{N}: N \geq 1\right)$, properties (2.9) and (2.10) hold for $\left\{\eta_{t}^{\mathcal{E}_{N}}: t \geq 0\right\}$ if and only if they do so for the speeded up process $\left\{\eta_{\theta_{N} t}^{\varepsilon_{N}}: t \geq 0\right\}$. Furthermore, condition (V1) remains invariant by any re-scale of time, while (5.1) and the assertion preceding it are implied by the corresponding claims for $\left\{\eta_{\theta_{N} t}^{\mathcal{E}_{N}}: t \geq 0\right\}$. In consequence, speeding up the process appropriately, we may assume in Proposition 5.1 that

$$
r_{N}\left(\mathcal{W}, \mathcal{B}^{c}\right)=1 \quad \forall N \geq 1
$$

and condition (2.10) becomes

$$
\lim _{N \rightarrow \infty} \sup _{\eta \in W_{N}} \mathbf{E}_{\eta}\left[T_{\boldsymbol{\xi}}(\mathcal{W})\right]=0 .
$$

To prove the last two assertions of Proposition 5.1, we show that the law of $T_{\mathcal{B}^{c}}(\mathcal{W})$ under $\mathbf{P}_{\eta^{N}}$ converges to a mean-one exponential distribution and that

$$
\lim _{N \rightarrow \infty} \mathbf{E}_{\eta^{N}}\left[T_{\mathcal{B}^{c}}(\mathcal{W})\right]=1 .
$$

We identify the trace process $\left\{\eta_{t}^{\mathcal{E}_{N}}: t \geq 0\right\}$ with the first marginal of the $\mathcal{E}_{N} \times\{0,1\}$-valued Markov process $\left\{\left(\eta_{t}^{\mathcal{E}_{N}}, X_{t}^{N}\right): t \geq 0\right\}$ defined as follows. To keep notation simple, let $\breve{x}=1-x, x=0,1$. The transition rates for $\left\{\left(\eta_{t}^{\mathcal{E}_{N}}, X_{t}^{N}\right): t \geq 0\right\}$ are the following:

- From each $(\eta, x) \in W_{N} \times\{0,1\}$, the process jumps to $(\xi, x)$ (resp. to $\left.(\xi, \breve{x})\right)$ with rate $R_{N}^{\mathcal{E}}(\eta, \xi)$ for any $\xi \in W_{N}$ (resp. for any $\xi \in B_{N}^{c}$ ).

- From each $(\eta, x) \in B_{N}^{c} \times\{0,1\}$, the process jumps to $(\xi, x)$ with rate $R_{N}^{\mathcal{E}}(\eta, \xi)$, for any $\xi \in \mathcal{E}_{N}$.

Let $\mathbf{P}_{(\eta, x)},(\eta, x) \in \mathcal{E}_{N} \times\{0,1\}$, be the probability measure on $D\left(\mathbb{R}_{+}, \mathcal{E}_{N} \times\{0,1\}\right)$ induced by the Markov process $\left\{\left(\eta_{t}^{\mathcal{E}_{N}}, X_{t}^{N}\right): t \geq 0\right\}$ starting from $(\eta, x)$. Hence, for any starting point $(\eta, x) \in \mathcal{E}_{N} \times\{0,1\}$, the law of the marginal $\left\{\eta_{t}^{\mathcal{E}_{N}}: t \geq 0\right\}$ on $D\left(\mathbb{R}_{+}, \mathcal{E}_{N}\right)$ under $\mathbf{P}_{(\eta, x)}$ is $\mathbf{P}_{\eta}$.

By Proposition 6.3 the conditioned probability measure $\mu_{N}^{\varepsilon}(\cdot):=\mu_{N}\left(\cdot \mid \varepsilon_{N}\right)$ is the invariant probability measure for the trace process $\left\{\eta^{\varepsilon_{N}}: t \geq 0\right\}$. Define the 
probability measure on $\mathcal{E}_{N} \times\{0,1\}$ by

$$
\mathfrak{m}_{N}(\eta, x)=(1 / 2) \mu_{N}^{\varepsilon}(\eta), \quad \text { for }(\eta, x) \in \mathcal{E}_{N} \times\{0,1\} .
$$

We may check that $\mathfrak{m}_{N}$ is an invariant probability measure for $\left\{\left(\eta_{t}^{\mathcal{E}_{N}}, X_{t}^{N}\right): t \geq 0\right\}$. In particular, $\left\{\left(\eta_{t}^{\mathcal{E}_{N}}, X_{t}^{N}\right): t \geq 0\right\}$ is positive recurrent.

Clearly, for any $\eta \in W_{N}$, the law of $T_{\mathcal{B}^{c}}(\mathcal{W})$ under $\mathbf{P}_{\eta}$ coincides with the law of the first jump

$$
\inf \left\{t>0: X_{t}^{N} \neq X_{0}^{N}\right\}
$$

under $\mathbf{P}_{(\eta, x)}$, for any $x \in\{0,1\}$. Hence, to prove that $T_{\mathcal{B}^{c}}(\mathcal{W})$ converges to a mean one exponential law it is enough to show that the second coordinate of the trace of the process $\left\{\left(\eta_{t}^{\mathcal{E}_{N}}, X_{t}^{N}\right): t \geq 0\right\}$ on $W_{N} \times\{0,1\}$ converges to a Markov process on $\{0,1\}$ which jumps from $x$ to $1-x$ at rate 1 . This is done in two steps. We first prove in Lemma 5.3 that the sequence of processes $\left\{X_{t}^{N}: t \geq 0\right\}$ is a tight family. Then, we characterize in Lemma 5.6 all limit points by showing that they solve a martingale problem. Both statements rely on a replacement result, stated in Lemma 5.2 and Lemma 5.5. which allows the substitution of a function by its conditional expectation.

Conditions (2.9) and (5.3) imply that

$$
\lim _{N \rightarrow \infty} \sup _{\eta \in W_{N}} \mathbf{E}_{\eta}\left[\int_{0}^{T_{\xi}(\mathcal{\varepsilon})}\left\{R_{N}^{\mathcal{W}}\left(\eta_{s}^{\mathcal{E}_{N}}\right)+1\right\} \mathbf{1}\left\{\eta_{s}^{\mathcal{E}_{N}} \in W_{N}\right\} d s\right]=0,
$$

where $T_{\boldsymbol{\xi}}(\mathcal{E})=T_{\boldsymbol{\xi}}^{N}(\mathcal{E}):=\inf \left\{t \geq 0: \eta_{t}^{\mathcal{E}_{N}}=\xi^{N}\right\}$. As a consequence of (5.5), we get the following lemma.

Lemma 5.2. For every $t>0$,

$$
\lim _{N \rightarrow+\infty} \sup _{\eta \in \mathcal{E}_{N}}\left|\mathbf{E}_{\eta}\left[\int_{0}^{t}\left(R_{N}^{\mathcal{W}}\left(\eta_{s}^{\mathcal{E}_{N}}\right)-1\right) \mathbf{1}\left\{\eta_{s}^{\mathcal{E}_{N}} \in W_{N}\right\} d s\right]\right|=0 .
$$

Proof. Recall the notation introduced in Subsection 6.2 Let $g: \mathcal{E}_{N} \rightarrow \mathbb{R}$ be given by $g(\eta)=R_{N}^{\mathcal{W}}(\eta) \mathbf{1}\left\{\eta \in W_{N}\right\}$ so that the expectation of $g$ with respect to $\mu_{N}^{\varepsilon}$ is equal to $\mu_{N}\left(W_{N}\right) / \mu_{N}\left(\mathcal{E}_{N}\right)$ in view of (5.2). Consider the partition $\pi=\left\{W_{N}, B_{N}^{c}\right\}$ of $\mathcal{E}_{N}$ and note that the conditional expectation $\langle g \mid \pi\rangle_{\mu_{N}^{\varepsilon}}=\mathbf{1}\left\{\eta \in W_{N}\right\}$. Since $g$ is integrable with respect to $\mu_{N}^{\varepsilon}$, the statement follows from (5.5) and Corollary 6.5 applied to the process $\left\{\eta_{t}^{\mathcal{E}_{N}}: t \geq 0\right\}$.

We use this lemma to show tightness for the sequence $\left\{X_{t}^{N}: t \geq 0\right\}$.

Lemma 5.3. Fix an arbitrary point $\boldsymbol{\eta}=\left(\eta^{N}: N \geq 1\right)$ in $\mathcal{W}$ and $z \in\{0,1\}$. For each $N \geq 1$, denote by $\mathbb{Q}_{N}$ the law of $\left\{X_{t}^{N}: t \geq 0\right\}$ under $\mathbf{P}_{\left(\eta^{N}, z\right)}$. Then the sequence of laws $\left(\mathbb{Q}_{N}: N \geq 1\right)$ is tight.

Proof. For each $T>0$, let $\mathfrak{T}_{T}$ denote the set of all stopping times bounded by $T$. By Aldous criterion (see Theorem 16.10 in [2]) we just need to show that

$$
\lim _{\delta \downarrow 0} \limsup _{N \rightarrow \infty} \sup _{\theta \leq \delta} \sup _{\tau \in \mathfrak{T}_{T}} \mathbf{P}_{\left(\eta^{N}, z\right)}\left[\left|X_{\tau+\theta}^{N}-X_{\tau}^{N}\right|>\epsilon\right]=0
$$

for every $\epsilon>0$ and $T>0$. Denote by $\mathbf{L}_{N}^{\mathcal{E}}$ the generator of $\left(\eta_{t}^{\mathcal{E}_{N}}, X_{t}^{N}\right)_{t \geq 0}$ and by $\mathbf{p}: \mathcal{E}_{N} \times\{0,1\} \rightarrow\{0,1\}$ the projection on the second coordinate. Consider the martingale

$$
M_{t}^{N}:=X_{t}^{N}-X_{0}^{N}-\int_{0}^{t}\left(\mathbf{L}_{N}^{\varepsilon} \mathbf{p}\right)\left(\eta_{s}^{\varepsilon_{N}}, X_{s}^{N}\right) d s .
$$


It is therefore enough to show that (5.6) holds with $X_{\tau+\theta}^{N}-X_{\tau}^{N}$ replaced by $M_{\tau+\theta}^{N}-M_{\tau}^{N}$ and by $\int_{\tau}^{\tau+\theta}\left(\mathbf{L}_{N}^{\mathcal{E}} \mathbf{p}\right)\left(\eta_{s}^{\mathcal{E}_{N}}, X_{s}^{N}\right) d s$.

Consider the integral term. By Chebychev inequality and by the strong Markov property, we need to prove that

$$
\lim _{\delta \downarrow 0} \limsup _{N \rightarrow \infty} \sup _{\theta \leq \delta} \sup _{(\eta, x) \in \mathcal{E}_{N} \times\{0,1\}} \mathbf{E}_{(\eta, x)}\left[\int_{0}^{\theta}\left|\left(\mathbf{L}_{N}^{\mathcal{E}} \mathbf{p}\right)\left(\eta_{s}^{\mathcal{E}_{N}}, X_{s}^{N}\right)\right| d s\right]=0,
$$

where $\mathbf{E}_{(\eta, x)}$ stands for the expectation with respect to $\mathbf{P}_{(\eta, x)}$. A simple computation provides

$$
\left(\mathbf{L}_{N}^{\mathcal{E}} \mathbf{p}\right)(\eta, x)=\{\breve{x}-x\} R_{N}^{\mathcal{W}}(\eta) \mathbf{1}\left\{\eta \in W_{N}\right\} .
$$

The proof is thus reduced to the claim

$$
\lim _{\delta \downarrow 0} \limsup _{N \rightarrow \infty} \sup _{\eta \in \mathcal{E}_{N}} \mathbf{E}_{\eta}\left[\int_{0}^{\delta} R_{N}^{\mathcal{W}}\left(\eta_{s}^{\mathcal{E}_{N}}\right) \mathbf{1}\left\{\eta_{s}^{\mathcal{E}_{N}} \in W_{N}\right\} d s\right]=0 .
$$

Since the expectation above is less than or equal to

$$
\left|\mathbf{E}_{\eta}\left[\int_{0}^{\delta}\left(R_{N}^{\mathcal{W}}\left(\eta_{s}^{\varepsilon_{N}}\right)-1\right) \mathbf{1}\left\{\eta_{s}^{\varepsilon_{N}} \in W_{N}\right\} d s\right]\right|+\delta,
$$

the limit (5.7) follows from Lemma 5.2.

We now turn to the martingale part $\left\{M_{t}^{N}: t \geq 0\right\}$, whose quadratic variation is given by

$$
\begin{aligned}
\langle M\rangle_{t}^{N} & =\int_{0}^{t}\left\{\mathbf{L}_{N}^{\mathcal{E}}\left(\mathbf{p}^{2}\right)-2 \mathbf{p} \mathbf{L}_{N}^{\mathcal{E}} \mathbf{p}\right\}\left(\eta_{s}^{\mathcal{E}_{N}}, X_{s}^{N}\right) d s \\
& =\int_{0}^{t} R_{N}^{\mathcal{W}}\left(\eta_{s}^{\mathcal{E}_{N}}\right) \mathbf{1}\left\{\eta_{s}^{\mathcal{E}_{N}} \in W_{N}\right\} d s .
\end{aligned}
$$

By Chebychev inequality

$$
\mathbf{P}_{\left(\eta^{N}, z\right)}\left[\left|M_{\tau+\theta}^{N}-M_{\tau}^{N}\right|>\epsilon\right] \leq \frac{1}{\epsilon^{2}} \mathbf{E}_{\left(\eta^{N}, z\right)}\left[\langle M\rangle_{\tau+\theta}^{N}-\langle M\rangle_{\tau}^{N}\right] .
$$

Finally, by the explicit formula for the quadratic variation and by the strong Markov property, the right hand side above is less than or equal to

$$
\frac{1}{\epsilon^{2}} \sup _{\eta \in \mathcal{E}_{N}} \mathbf{E}_{\eta}\left[\int_{0}^{\delta} R_{N}^{\mathcal{W}}\left(\eta_{s}^{\mathcal{E}_{N}}\right) \mathbf{1}\left\{\eta_{s}^{\mathcal{E}_{N}} \in W_{N}\right\} d s\right] \text {. }
$$

It remains to use (5.7).

As a consequence of Lemma 5.3 we obtain condition $(\mathbf{V 1})$ for the triple $(\mathcal{W}, \mathcal{W}, \boldsymbol{\xi})$ with respect to the trace process $\left\{\eta_{t}^{\mathcal{E}_{N}}: t \geq 0\right\}$.

Lemma 5.4. For any point $\boldsymbol{\eta}=\left(\eta^{N}: N \geq 1\right)$ in $\mathcal{W}$,

$$
\lim _{N \rightarrow+\infty} \mathbf{P}_{\eta^{N}}\left[T_{\boldsymbol{\xi}}(\mathcal{W})<T_{\mathcal{B}^{c}}(\mathcal{W})\right]=1
$$

Proof. Fix $\eta^{N}$ in $W_{N}, N \geq 1$. Consider the modified uniform modulus of continuity $\omega_{\delta}^{\prime}: D\left(\mathbb{R}_{+},\{0,1\}\right) \rightarrow \mathbb{R}_{+}$given by

$$
\omega_{\delta}^{\prime}(x .):=\inf _{\left\{t_{i}\right\}} \max _{0 \leq i<r} \sup _{t_{i} \leq s<t<t_{i+1}}\left|x_{t}-x_{s}\right|,
$$


where the first infimum is taken over all partitions $\left\{t_{i}: 0 \leq i \leq r\right\}$ of the interval $[0,1]$ such that

$$
\left\{\begin{array}{l}
0=t_{0}<t_{1}<\cdots<t_{r}=T \\
t_{i}-t_{i-1}>\delta, \quad \text { for } i=1, \ldots, r .
\end{array}\right.
$$

By the previous lemma (see e.g. Theorem 1.3 in Chapter 4 of [15]),

$$
\lim _{\delta \downarrow 0} \limsup _{N \rightarrow+\infty} \mathbf{P}_{\left(\eta^{N}, 0\right)}\left[\omega_{\delta}^{\prime}\left(X_{\cdot}^{N}\right)=1\right]=0 .
$$

Therefore, since for all $\delta>0\left\{T_{\mathcal{B}^{c}}(\mathcal{W}) \leq \delta\right\} \subset\left\{\omega_{\delta}^{\prime}\left(X_{\cdot}^{N}\right)=1\right\} \mathbf{P}_{\left(\eta^{N}, z\right)}$-almost surely,

$$
\lim _{\delta \downarrow} \liminf _{N \rightarrow+\infty} \mathbf{P}_{\eta^{N}}\left[T_{\mathcal{B}^{c}}(\mathcal{W})>\delta\right]=1 .
$$

On the other hand, by (5.3), we have

$$
\lim _{N \rightarrow+\infty} \mathbf{P}_{\eta^{N}}\left[T_{\boldsymbol{\xi}}(\mathcal{W})>\delta\right]=0
$$

for any $\delta>0$. The desired result follows from (5.9) and (5.8).

Actually, since $\left\{T_{\boldsymbol{\xi}}(\mathcal{W})<T_{\mathcal{B}^{c}}(\mathcal{W})\right\} \subseteq\left\{T_{\boldsymbol{\xi}}<T_{\mathcal{B}^{c}}\right\}$, Lemma 5.4 proves condition (V1) for the triple $(\mathcal{W}, \mathcal{B}, \boldsymbol{\xi})$ with respect to the process $\left\{\eta_{t}^{N} ; t \geq 0\right\}$ : For any point $\boldsymbol{\eta}=\left(\eta^{N}: N \geq 1\right)$ in $\mathcal{W}$,

$$
\lim _{N \rightarrow+\infty} \mathbf{P}_{\eta^{N}}\left[T_{\boldsymbol{\xi}}<T_{\mathcal{B}^{c}}\right]=1 .
$$

We now consider the trace of $\left\{\left(\eta_{t}^{\mathcal{E}_{N}}, X_{t}^{N}\right): t \geq 0\right\}$ on $W_{N} \times\{0,1\}$, denoted by $\left\{\left(\eta_{t}^{W_{N}}, X_{t}^{W_{N}}\right): t \geq 0\right\}$. As we shall see in Section [6. since $\left\{\left(\eta_{t}^{\varepsilon_{N}}, X_{t}^{N}\right): t \geq 0\right\}$ is positive recurrent, the trace process $\left\{\left(\eta_{t}^{W_{N}}, X_{t}^{W_{N}}\right): t \geq 0\right\}$ is positive recurrent as well. Moreover, the invariant probability measure for the trace process, denoted by $\mathfrak{m}_{N}^{\mathcal{W}}$, coincides with $\mathfrak{m}_{N}$ conditioned to $W_{N} \times\{0,1\}$ :

$$
\mathfrak{m}_{N}^{\mathcal{W}}(\eta, x):=(1 / 2) \mu_{N}^{\mathcal{W}}(\eta), \quad \text { for }(\eta, x) \in W_{N} \times\{0,1\} .
$$

The marginal process $\left\{\eta_{t}^{W_{N}}: t \geq 0\right\}$ corresponds to the trace of $\left\{\eta_{t}^{\mathcal{E}_{N}}: t \geq 0\right\}$ on $W_{N}$.

Let $\mathbf{L}_{N}^{\mathcal{W}}$ denote the Markov generator of $\left\{\left(\eta_{t}^{W_{N}}, X_{t}^{W_{N}}\right): t \geq 0\right\}$. Define, in addition, the Markov generator $\mathcal{L}$ as

$$
\mathcal{L} F(x):=F(\breve{x})-F(x), \quad x \in\{0,1\},
$$

for every $F:\{0,1\} \rightarrow \mathbb{R}$. For each $N \geq 1$, let $\mathbf{p}=\mathbf{p}_{N}$ be the projection function on the second coordinate $\mathbf{p}: W_{N} \times\{0,1\} \rightarrow\{0,1\}$. If $\mathbf{R}_{N}^{\mathcal{W}}(\cdot, \cdot)$ stands for the transition rates of $\left\{\left(\eta_{t}^{W_{N}}, X_{t}^{W_{N}}\right): t \geq 0\right\}$, we have that

$$
\mathbf{L}_{N}^{\mathcal{W}}(F \circ \mathbf{p})(\eta, x)=\{F(\breve{x})-F(x)\} \sum_{\xi \in W_{N}} \mathbf{R}_{N}^{\mathcal{W}}((\eta, x),(\xi, \breve{x}))
$$

for any $(\eta, x) \in W_{N} \times\{0,1\}$. By applying Corollary 6.2 to the Markov process $\left\{\left(\eta_{t}^{\mathcal{E}_{N}}, X_{t}^{N}\right): t \geq 0\right\}$ and its trace on $W_{N} \times\{0,1\}$, we get that

$$
\sum_{\xi \in W_{N}} \mathbf{R}_{N}^{\mathcal{W}}((\eta, x),(\xi, \breve{x}))=R_{N}^{\mathcal{W}}(\eta)
$$

for all $(\eta, x) \in W_{N} \times\{0,1\}$. Therefore, by (5.2), the conditional expectation of $\mathbf{L}_{N}^{\mathcal{W}}(F \circ \mathbf{p})$, under $\mathfrak{m}_{N}^{\mathcal{W}}$, given the $\sigma$-field generated by the partition

$$
W_{N} \times\{0,1\}=\left(W_{N} \times\{0\}\right) \cup\left(W_{N} \times\{1\}\right),
$$


is $(\mathcal{L} F) \circ \mathbf{p}$. Therefore, applying Corollary 6.11 to the trace process $\left\{\left(\eta_{t}^{W_{N}}, X_{t}^{W_{N}}\right)\right.$ : $t \geq 0\}$, the function $\mathbf{L}_{N}^{\mathcal{W}}(F \circ \mathbf{p})$ and the partition (5.11), we obtain the following replacement lemma.

Lemma 5.5. For every $x \in\{0,1\}$, function $F:\{0,1\} \rightarrow \mathbb{R}$ and time $t>0$,

$$
\lim _{N \rightarrow \infty} \sup _{\eta \in W_{N}}\left|\mathbf{E}_{(\eta, x)}\left[\int_{0}^{t}\left\{\mathbf{L}_{N}^{\mathcal{W}}(F \circ \mathbf{p})\left(\eta_{s}^{W_{N}}, X_{s}^{W_{N}}\right)-\mathcal{L} F\left(X_{s}^{W_{N}}\right)\right\} d s\right]\right|=0 .
$$

Proof. Recall that the conditional expectation of $\mathbf{L}_{N}^{\mathcal{W}}(F \circ \mathbf{p})$ is $(\mathcal{L} F) \circ \mathbf{p}$. Since

$$
\left|\mathbf{L}_{N}^{\mathcal{W}}(F \circ \mathbf{p})(\eta, x)-\mathcal{L} F(x)\right| \leq\left(R_{N}^{\mathcal{W}}(\eta)+1\right) \max \{|F(0)|,|F(1)|\},
$$

in view of Corollary 6.5, to prove the lemma we just need to check that for any $x \in\{0,1\}$,

$$
\lim _{N \rightarrow \infty} \sup _{\eta \in W_{N}} \mathbf{E}_{(\eta, x)}\left[\int_{0}^{\mathbf{T}_{(\xi, x)}^{\mathcal{W}}}\left(R_{N}^{\mathcal{W}}\left(\eta_{s}^{W_{N}}\right)+1\right) \mathbf{1}\left\{X_{s}^{W_{N}}=x\right\} d s\right]=0,
$$

where, for each $N \geq 1$,

$$
\mathbf{T}_{(\boldsymbol{\xi}, x)}^{\mathcal{W}}=\mathbf{T}_{(\boldsymbol{\xi}, x)}^{\mathcal{W}}(N):=\inf \left\{t \geq 0:\left(\eta_{t}^{W_{N}}, X_{t}^{W_{N}}\right)=\left(\xi^{N}, x\right)\right\} .
$$

Fix an arbitrary $x \in\{0,1\}$. It follows from conditions (2.9) and (5.3) that

$$
\lim _{N \rightarrow \infty} \sup _{\eta \in W_{N}} \mathbf{E}_{\eta}\left[\int_{0}^{T_{\boldsymbol{\xi}}(\mathcal{W})}\left(R_{N}^{\mathcal{W}}\left(\eta_{s}^{W_{N}}\right)+1\right) d s\right]=0 .
$$

To keep notation simple, let us denote

$$
\mathbf{T}_{N}:=\int_{0}^{\mathbf{T}_{(\xi, x)}^{\mathcal{W}}}\left(R_{N}^{\mathcal{W}}\left(\eta_{s}^{W_{N}}\right)+1\right) \mathbf{1}\left\{X_{s}^{W_{N}}=x\right\} d s .
$$

Since $\left\{T_{\boldsymbol{\xi}}(\mathcal{W})<T_{\mathcal{B}^{c}}(\mathcal{W})\right\} \subseteq\left\{T_{\boldsymbol{\xi}}(\mathcal{W})=\mathbf{T}_{(\boldsymbol{\xi}, x)}^{\mathcal{W}}\right\}$, by Lemma 5.4, (5.13) and Chebychev inequality, for every $t>0$,

$$
\lim _{N \rightarrow \infty} \sup _{\eta \in W_{N}} \mathbf{P}_{(\eta, x)}\left[\mathbf{T}_{N}>t\right]=0 .
$$

By the strong Markov property, (5.14) and the arguments presented in the proof of Lemma 4.3 .

$$
\lim _{A \rightarrow \infty} \limsup _{N \rightarrow \infty} \sup _{\eta \in W_{N}} \mathbf{E}_{(\eta, x)}\left[\mathbf{T}_{N} \mathbf{1}\left\{\mathbf{T}_{N}>A\right\}\right]=0 .
$$

Hence, by Lemma 5.4.

$$
\limsup _{N \rightarrow \infty} \sup _{\eta \in W_{N}} \mathbf{E}_{(\eta, x)}\left[\mathbf{T}_{N} \mathbf{1}\left\{T_{\boldsymbol{\xi}}(\mathcal{W}) \geq T_{\mathcal{B}^{c}}(\mathcal{W})\right\}\right]=0 .
$$

On the other hand,

$$
\mathbf{E}_{(\eta, x)}\left[\mathbf{T}_{N} \mathbf{1}\left\{T_{\boldsymbol{\xi}}(\mathcal{W})<T_{\mathcal{B}^{c}}(\mathcal{W})\right\}\right] \leq \mathbf{E}_{\eta}\left[\int_{0}^{T_{\boldsymbol{\xi}}(\mathcal{W})}\left(R_{N}^{\mathcal{W}}\left(\eta_{s}^{W_{N}}\right)+1\right) d s\right]
$$

Therefore, (5.12) follows from this estimate, (5.13) and (5.15).

We now prove the convergence in law of $\left\{X_{t}^{W_{N}}: t \geq 0\right\}$ as $N \uparrow \infty$. Fix an arbitrary point $\boldsymbol{\eta}=\left(\eta^{N}: N \geq 1\right)$ in $\mathcal{W}$. For each $N \geq 1$, denote by $\mathbb{P}_{N}$ the law of $\left\{X_{t}^{W_{N}}: t \geq 0\right\}$ under $\mathbf{P}_{\left(\eta^{N}, 0\right)}$. Following the same argument presented in the proof of Lemma 5.3 we can show that $\left(\mathbb{P}_{N}: N \geq 1\right)$ is tight. 
The uniqueness of limit points for this sequence is established as follows. Assume without loss of generality that $\mathbb{P}_{N} \rightarrow \mathbb{P}$, as $N \rightarrow \infty$, for some probability measure $\mathbb{P}$ on $D\left(\mathbb{R}_{+},\{0,1\}\right)$. For $t \geq 0$, let $X_{t}$ denote the time-projection $X_{t}: D\left(\mathbb{R}_{+},\{0,1\}\right) \rightarrow$ $\{0,1\}$. We shall prove in the following lemma that $\mathbb{P}$ solves the martingale problem associated to the generator $\mathcal{L}$ defined in (5.10). It is well known that this property together with the distribution of $X_{0}$, characterize the measure $\mathbb{P}$.

Lemma 5.6. Under $\mathbb{P}, X_{0}=0$ a.s. and

$$
M_{t}^{F}=F\left(X_{t}\right)-F\left(X_{0}\right)-\int_{0}^{t} \mathcal{L} F\left(X_{s}\right) d s, \quad \text { for } t \geq 0,
$$

is a martingale for any function $F:\{0,1\} \rightarrow \mathbb{R}$.

Proof. The first claim is trivial. For the last one, fix $0 \leq s<t$, a function $F$ : $\{0,1\} \mapsto \mathbb{R}$ and a bounded function $U: D\left(\mathbb{R}_{+},\{0,1\}\right) \mapsto \mathbb{R}$ depending only on $\left\{X_{r}: 0 \leq r \leq s\right\}$ and continuous for the Skorohod topology. Denote by $\mathbb{E}$ and $\mathbb{E}_{N}$ the expectation with respect to $\mathbb{P}$ and $\mathbb{P}_{N}$, respectively. We shall prove that

$$
\mathbb{E}\left[M_{t}^{F} U\right]=\mathbb{E}\left[M_{s}^{F} U\right] .
$$

Recall that $\mathbf{L}_{N}^{\mathcal{W}}$ denotes the generator of $\left\{\left(\eta_{t}^{W_{N}}, X_{t}^{W_{N}}\right): t \geq 0\right\}$. For $N \geq 1$, consider the $\mathbf{P}_{\left(\eta^{N}, 0\right)}$-martingale $\left\{M_{t}^{N}: t \geq 0\right\}$, defined by

$$
M_{t}^{N}=F\left(X_{t}^{W_{N}}\right)-F(0)-\int_{0}^{t} \mathbf{L}_{N}^{\mathcal{W}}(F \circ \mathbf{p})\left(\eta_{s}^{W_{N}}, X_{s}^{W_{N}}\right) d s, \quad t \geq 0 .
$$

Denote $U^{N}:=U\left(X^{W_{N}}\right)$. As $\left\{M_{t}^{N}: t \geq 0\right\}$ is a martingale,

$$
\mathbf{E}_{\left(\eta^{N}, 0\right)}\left[M_{t}^{N} U^{N}\right]=\mathbf{E}_{\left(\eta^{N}, 0\right)}\left[M_{s}^{N} U^{N}\right]
$$

so that

$$
\mathbf{E}_{\left(\eta^{N}, 0\right)}\left[U^{N}\left\{F\left(X_{t}^{W_{N}}\right)-F\left(X_{s}^{W_{N}}\right)-\int_{s}^{t} \mathbf{L}_{N}^{\mathcal{W}}(F \circ \mathbf{p})\left(\eta_{r}^{W_{N}}, X_{r}^{W_{N}}\right) d r\right\}\right]=0 .
$$

On the other hand, since $U^{N}$ is bounded and $\mathcal{F}_{s}$-measurable, it follows from the Markov property and Lemma 5.5 that

$$
\lim _{N \rightarrow \infty} \mathbf{E}_{\left(\eta^{N}, 0\right)}\left[U^{N} \int_{s}^{t}\left\{\mathbf{L}_{N}^{\mathcal{W}}(F \circ \mathbf{p})\left(\eta_{r}^{W_{N}}, X_{r}^{W_{N}}\right)-\mathcal{L} F\left(X_{r}^{W_{N}}\right)\right\} d r\right]=0 .
$$

Putting the last two assertions together we get

$$
\lim _{N \rightarrow \infty} \mathbb{E}_{N}\left[U\left\{F\left(X_{t}\right)-F\left(X_{s}\right)-\int_{s}^{t} \mathcal{L} F\left(X_{r}\right) d r\right\}\right]=0 .
$$

Now, since $\mathbb{P}_{N}$ converges to $\mathbb{P}$, time averages of $\mathbb{E}_{N}\left[F\left(X_{t}\right) U\right]$ and $\mathbb{E}_{N}\left[F\left(X_{s}\right) U\right]$ converge to time averages of $\mathbb{E}\left[F\left(X_{t}\right) U\right]$ and $\mathbb{E}\left[F\left(X_{s}\right) U\right]$, respectively. Hence, from this last observation and (5.17) it follows that

$$
\frac{1}{\epsilon} \int_{0}^{\epsilon} d r \mathbb{E}\left[U\left\{F\left(X_{t+r}\right)-F\left(X_{s+r}\right)-\int_{s+r}^{t+r} \mathcal{L} F\left(X_{s}\right)\right\}\right]=0
$$

for every $\epsilon>0$. It remains to let $\epsilon \downarrow 0$ and use the right continuity of the process to deduce (5.16), which concludes the proof of the lemma. 
Under $\mathbb{P},\left\{X_{t}: t \geq 0\right\}$ is therefore a Markov chain on $\{0,1\}$ with generator $\mathcal{L}$ and starting at 0 . We have thus shown that, the law of

$$
T_{\mathcal{B}^{c}}(\mathcal{W})=\inf \left\{t>0: X_{t}^{W_{N}}=1\right\},
$$

under $\mathbf{P}_{\left(\eta^{N}, 0\right)}$, converges to a mean-one exponential distribution. To conclude the proof of Proposition 5.1 it remains to check (5.4). By Lemma 5.4 and the convergence in law of $T_{\mathcal{B}^{c}}(\mathcal{W}),(\mathcal{W}, \mathcal{W}, \boldsymbol{\xi})$ is a valley for the trace process $\left\{\eta_{t}^{\mathcal{E}_{N}}: t \geq 0\right\}$ with depth 1. Therefore, applying item $(i i)$ of Proposition 4.4 to $(\mathcal{W}, \mathcal{W}, \boldsymbol{\xi})$ and $\left\{\eta_{t}^{\mathcal{E}_{N}}: t \geq 0\right\}$ we get (5.4). This concludes the proof of Proposition 5.1

We are now in a position to prove Theorem 2.7 Condition (V1) follows from Proposition 5.1 and Condition (V3) from (2.11) and Chebychev inequality. Condition (V2) follows from (V3) and the convergence in law of $r_{N}\left(\mathcal{W}, \mathcal{B}^{c}\right) T_{\mathcal{B}^{c}}(\mathcal{W})$ stated in Proposition [5.1.

Proof of Theorem 2.8. Next result is the main step in the proof of Theorem 2.8

Proposition 5.7. Recall the notation introduced in Subsection 2.2. If conditions (C2), (C3) and (H0) are in force, then so are (M1) and (M2).

The proof of this result is divided in three lemmas. As in the proof of Proposition 5.1. without loss of generality, we may assume that $\theta_{N}=1, \forall N \geq 1$. In this way, condition (H0) guarantees that, for every $x, y \in S, x \neq y$,

$$
\lim _{N \rightarrow \infty} r_{N}\left(\mathcal{E}^{x}, \mathcal{E}^{y}\right)=r(x, y),
$$

and we shall prove the convergence in law of the sequence $\left\{X_{t}^{N}: t \geq 0\right\}, N \geq 0$.

Clearly, conditions (C2) and (C3) imply

$$
\lim _{N \rightarrow \infty} \sup _{\eta \in \mathcal{E}_{N}^{x}} \mathbf{E}_{\eta}\left[\int_{0}^{T_{\boldsymbol{\xi}_{x}}}\left\{R_{N}^{x}\left(\eta_{s}^{\mathcal{E}_{N}}\right)+r_{N}\left(\mathcal{E}^{x}, \breve{\mathcal{E}}^{x}\right)\right\} \mathbf{1}\left\{\eta_{s}^{\mathcal{E}_{N}} \in \mathcal{E}_{N}^{x}\right\} d s\right]=0
$$

for any $x \in S$, where $\left\{\eta_{t}^{\varepsilon_{N}}: t \geq 0\right\}$ stands for the trace process of $\left\{\eta_{t}: t \geq 0\right\}$ on $\mathcal{E}_{N}$. Let us define $V_{N}: \mathcal{E}_{N} \mapsto \mathbb{R}$ as

$$
V_{N}(\eta):=\sum_{x \in S} R_{N}^{x}(\eta) \mathbf{1}\left\{\eta \in \mathcal{E}_{N}^{x}\right\}, \quad \eta \in \mathcal{E}_{N} .
$$

Let $\mu_{N}^{\varepsilon}$ be the measure $\mu_{N}$ conditioned to $\varepsilon_{N}$ and denote by $\hat{V}_{N}$ the $\mu_{N}^{\varepsilon}$-conditional expectation of $V_{N}$ given the $\sigma$-algebra generated by the partition $\mathcal{E}_{N}=\cup_{x \in S} \mathcal{E}_{N}^{x}$ :

$$
\hat{V}_{N}(\eta):=\sum_{x \in S} r_{N}\left(\mathcal{E}^{x}, \breve{\mathcal{E}}^{x}\right) \mathbf{1}\left\{\eta \in \mathcal{E}_{N}^{x}\right\}, \quad \forall \eta \in \mathcal{E}_{N} .
$$

Since $V_{N}$ is integrable with respect to $\mu_{N}^{\varepsilon}$, it follows from Corollary 6.5 and from (5.19) that, for any $t>0$,

$$
\lim _{N \rightarrow \infty} \sup _{\eta \in \mathcal{E}_{N}}\left|\mathbf{E}_{\eta}\left[\int_{0}^{t}\left\{V_{N}-\hat{V}_{N}\right\}\left(\eta_{s}^{\mathcal{E}_{N}}\right) d s\right]\right|=0 .
$$

In order to prove (M2), fix some $x \in S$ and a point $\boldsymbol{\eta}=\left(\eta^{N}: N \geq 1\right)$ in $\mathcal{E}^{x}$. For each $N \geq 1$, denote by $\mathbb{P}_{N}$ the law of $\left\{X_{t}^{N}: t \geq 0\right\}$ under $\mathbf{P}_{\eta^{N}}$. The convergence of the sequence $\left(\mathbb{P}_{N}: N \geq 1\right)$ stated in (M2), follows from tightness and uniqueness of limit points. We first examine the tightness.

Lemma 5.8. The sequence $\left(\mathbb{P}_{N}: N \geq 1\right)$ is tight. 
Proof. For each $T>0$, let $\mathfrak{T}_{T}$ denote the set of all stopping times bounded by $T$. By Aldous criterion (see Theorem 16.10 in [2]) we just need to show that

$$
\lim _{\delta \downarrow 0} \lim _{N \rightarrow \infty} \sup _{\theta \leq \delta} \sup _{\tau \in \mathfrak{T}_{T}} \mathbf{P}_{\eta_{N}}\left[\left|X_{\tau+\theta}^{N}-X_{\tau}^{N}\right|>\epsilon\right]=0
$$

for every $\epsilon>0$ and $T>0$.

Let $L_{N}^{\mathcal{E}}$ be the generator of the trace process $\left\{\eta_{t}^{\mathcal{E}_{N}}: t \geq 0\right\}$ and let $\left\{M_{t}^{N}: t \geq 0\right\}$ be the martingale defined by

$$
M_{t}^{N}=X_{t}^{N}-X_{0}^{N}-\int_{0}^{t} L_{N}^{\varepsilon} \Psi_{N}\left(\eta_{s}^{\varepsilon_{N}}\right) d s
$$

To prove tightness, it is therefore enough to show that (5.21) holds with the difference $X_{\tau+\theta}^{N}-X_{\tau}^{N}$ replaced by $M_{\tau+\theta}^{N}-M_{\tau}^{N}$ and by $\int_{\tau}^{\tau+\theta} L_{N}^{\varepsilon} X_{s}^{N} d s$.

Consider the integral term. By Chebychev inequality and by the strong Markov property, we need to prove that

$$
\lim _{\delta \downarrow 0} \lim _{N \rightarrow \infty} \sup _{\theta \leq \delta} \sup _{\eta \in \varepsilon_{N}} \mathbf{E}_{\eta}\left[\int_{0}^{\theta}\left|L_{N}^{\varepsilon} \Psi_{N}\left(\eta_{s}^{\varepsilon_{N}}\right)\right| d s\right]=0 .
$$

An elementary computation shows that

$$
L_{N}^{\varepsilon} \Psi_{N}(\eta)=\sum_{x, y \in S}\{y-x\} R_{N}^{x, y}(\eta) \mathbf{1}\left\{\eta \in \mathcal{E}_{N}^{x}\right\},
$$

for any $\eta \in \mathcal{E}_{N}$. Since $\left|L_{N}^{\mathcal{E}} \Psi_{N}\right| \leq \kappa V_{N}$, the proof is reduced to the claim

$$
\lim _{\delta \downarrow 0} \lim _{N \rightarrow \infty} \sup _{\eta \in \mathcal{E}_{N}} \mathbf{E}_{\eta}\left[\int_{0}^{\delta} V_{N}\left(\eta_{s}^{\varepsilon_{N}}\right) d s\right]=0 .
$$

The left hand side can be written as

$$
\lim _{\delta \downarrow 0} \lim _{N \rightarrow \infty} \sup _{\eta \in \mathcal{E}_{N}}\left\{\mathbf{E}_{\eta}\left[\int_{0}^{\delta}\left\{V_{N}-\hat{V}_{N}\right\}\left(\eta_{s}^{\varepsilon_{N}}\right) d s\right]+\mathbf{E}_{\eta}\left[\int_{0}^{\delta} \hat{V}_{N}\left(\eta_{s}^{\varepsilon_{N}}\right) d s\right]\right\} .
$$

The first term converges to zero as $N \uparrow \infty$, for any $\delta>0$, by (5.20). The second term is bounded above by

$$
\lim _{\delta \downarrow 0} \lim _{N \rightarrow \infty} \delta \sum_{x \in S} r_{N}\left(\mathcal{E}_{N}^{x}, \breve{\mathcal{E}}_{N}^{x}\right),
$$

which is equal to zero by (5.18).

We now turn to the martingale part, whose quadratic variation, denoted by $\left\langle M^{N}\right\rangle_{t}$, is given by

$$
\left\langle M^{N}\right\rangle_{t}=\int_{0}^{t}\left\{L_{N}^{\varepsilon}\left(\Psi_{N}\right)^{2}\left(\eta_{s}^{\mathcal{E}_{N}}\right)-2 X_{s}^{N}\left(L_{N}^{\varepsilon} \Psi_{N}\right)\left(\eta_{s}^{\mathcal{E}_{N}}\right)\right\} d s, \quad t \geq 0 .
$$

An elementary computation shows that this expression is equal to

$$
\sum_{x, y \in S}\{y-x\}^{2} R_{N}^{x, y}(\eta) \mathbf{1}\left\{\eta \in \mathcal{E}_{N}^{x}\right\} .
$$


By the explicit formula for the quadratic variation, by Chebychev inequality and by the strong Markov property,

$$
\begin{aligned}
\mathbf{P}_{\eta^{N}}\left[\left|M_{\tau+\theta}^{N}-M_{\tau}^{N}\right|>\epsilon\right] & \leq \frac{1}{\epsilon^{2}} \mathbf{E}_{\eta^{N}}\left[\left\langle M^{N}\right\rangle_{\tau+\theta}-\left\langle M^{N}\right\rangle_{\tau}\right] \\
& \leq \frac{\kappa^{2}}{\epsilon^{2}} \sup _{\eta \in \mathcal{E}_{N}} \mathbf{E}_{\eta}\left[\int_{0}^{\delta} V_{N}\left(\eta_{s}^{\mathcal{E}_{N}}\right) d s\right] .
\end{aligned}
$$

It remains to repeat the arguments presented for the integral term of the decomposition.

Now we turn to the uniqueness of limit points. Assume without loss of generality that the sequence $\mathbb{Q}_{N}$ converges to a measure $\mathbb{P}$. Denote by $\mathfrak{L}_{N}$ and $\mathfrak{L}$ the Markov generators on the state space $S=\{1, \ldots, \kappa\}$ given by

$$
\left(\mathfrak{L}_{N} F\right)(x)=\sum_{y \in S \backslash\{x\}}\{F(y)-F(x)\} r_{N}\left(\mathcal{E}^{x}, \mathcal{E}^{y}\right)
$$

and

$$
(\mathfrak{L} F)(x)=\sum_{y \in S \backslash\{x\}}\{F(y)-F(x)\} r(x, y) .
$$

For $t \geq 0$, let $X_{t}$ denote the projection $D\left(\mathbb{R}_{+}, S\right) \mapsto S$. The probability $\mathbb{P}$ is completely determined by the properties stated in the following lemma.

Lemma 5.9. Under $\mathbb{P}, X_{0}=x$ and

$$
M_{t}=F\left(X_{t}\right)-F\left(X_{0}\right)-\int_{0}^{t} \mathfrak{L} F\left(X_{s}\right) d s
$$

is a martingale for any function $F: S \mapsto \mathbb{R}$.

The proof of this lemma follows closely the one of Lemma 5.6 It suffices, in particular, to show the following replacement lemma. Let $L_{N}^{\mathcal{E}}$ stand for the generator of $\left\{\eta_{t}^{\mathcal{E}_{N}}: t \geq 0\right\}$.

Lemma 5.10. For any $t>0$,

$$
\lim _{N \rightarrow \infty} \sup _{\eta \in \mathcal{E}_{N}} \mathbf{E}_{\eta}\left[\int_{0}^{t}\left\{L_{N}^{\varepsilon}\left(F \circ \Psi_{N}\right)-(\mathfrak{L} F) \circ \Psi_{N}\right\}\left(\eta_{s}^{\varepsilon_{N}}\right) d s\right]=0 .
$$

Proof. First, by condition (H0), we have that

$$
\lim _{N \rightarrow \infty} \sup _{\eta \in \mathcal{E}_{N}} \mathbf{E}_{\eta}\left[\int_{0}^{t}\left\{\left(\mathfrak{L}_{N} F\right)\left(X_{s}^{N}\right)-(\mathfrak{L} F)\left(X_{s}^{N}\right)\right\} d s\right]=0 .
$$

It remains to prove that

$$
\lim _{N \rightarrow \infty} \sup _{\eta \in \mathcal{E}_{N}} \mathbf{E}_{\eta}\left[\int_{0}^{t}\left\{L_{N}^{\varepsilon}\left(F \circ \Psi_{N}\right)-\left(\mathfrak{L}_{N} F\right) \circ \Psi_{N}\right\}\left(\eta_{s}^{\mathcal{E}_{N}}\right) d s\right]=0 .
$$

The $\mu_{N}^{\mathcal{E}}$-conditional expectation of $L_{N}^{\mathcal{E}}\left(F \circ \Psi_{N}\right)$ given the $\sigma$-algebra generated by the partition $\mathcal{E}_{N}=\cup_{x \in S} \mathcal{E}_{N}^{x}$ is $\left(\mathfrak{L}_{N} F\right) \circ \Psi_{N}$. The expectation of $\left|L_{N}^{\mathcal{E}}\left(F \circ \Psi_{N}\right)\right|$ with respect to $\mu_{N}^{\mathcal{E}}$ is bounded by $C(F) \sum_{x \in S} r_{N}\left(\mathcal{E}^{x}, \mathcal{E}^{y}\right)$ for some finite constant $C(F)$, depending only on $F$, and, for any $\eta \in \mathcal{E}_{N},\left|L_{N}^{\mathcal{E}}\left(F \circ \Psi_{N}\right)(\eta)-\left(\mathfrak{L}_{N} F\right) \circ \Psi_{N}(\eta)\right|$ is bounded above by

$$
2 \max _{z \in S}|F(z)| \sum_{x \in S}\left\{R_{N}^{x}(\eta)-r_{N}\left(\mathcal{E}^{x}, \mathcal{E}^{y}\right)\right\} \mathbf{1}\left\{\eta \in \mathcal{E}_{N}^{x}\right\} .
$$


By Corollary 6.5, applied to $g=L_{N}^{\varepsilon}\left(F \circ \Psi_{N}\right)$ and by (5.19), (5.23) holds, which concludes the proof of the lemma.

This concludes the proof of condition (M2). Condition (M1) follows from Proposition 5.1 with $\mathcal{W}=\mathcal{E}^{x}, \mathcal{B}=\mathcal{E}^{x} \cup \boldsymbol{\Delta}$, which concludes the proof of Proposition 5.7.

For Theorem 2.8 it remains to check condition (M3) for non-absorbing states. This follows from Proposition 2.4 since condition (M2) has already been deduced.

Proof of Theorem 2.9. We assume in this subsection that the process is reversible and adopt all notation introduced in Section 2 The proof of Theorem 2.9 relies on the following result which states the important fact that, under condition (2.15), the capacity between $\mathcal{W}$ and $\mathcal{B}^{c}$ is asymptotically equivalent to the capacity between any point $\boldsymbol{\zeta}$ of $\mathcal{W}$ and $\mathcal{B}^{c}$.

Proposition 5.11. Consider two sequences of sets $\mathcal{W}$ and $\mathcal{B}$ satisfying (2.3). Assume that condition (2.15) holds for some point $\boldsymbol{\xi}=\left(\xi^{N}: N \geq 1\right)$ in $\mathcal{W}$. Then, the assertions of Proposition 5.1 are in force. Moreover, for every point $\zeta=\left(\zeta^{N}: N \geq 1\right)$ in $\mathcal{W}$,

$$
\lim _{N \rightarrow \infty} \frac{\operatorname{cap}_{N}\left(\mathcal{W}, \mathcal{B}^{c}\right)}{\operatorname{cap}_{N}\left(\boldsymbol{\zeta}, \mathcal{B}^{c}\right)}=1
$$

and

$$
\lim _{N \rightarrow \infty} \inf _{\eta \in W_{N}} \mathbf{P}_{\eta}\left[T_{\zeta}<T_{\mathcal{B}^{c}}\right]=1
$$

Proof. We have shown just before the statement of Theorem 2.9 that conditions (2.9), (2.10) follow from (2.15). In particular, the assertions of Proposition 5.1 hold.

Fix an arbitrary point $\zeta=\left(\zeta^{N}: N \geq 1\right)$ in $\mathcal{W}$. By (6.16) applied to $\left\{\eta^{N}\right\}$, $g=\mathbf{1}\{\mathcal{W}\},\left\{\xi^{N}\right\}$, and to $\left\{\xi^{N}\right\}, g=\mathbf{1}\{\mathcal{W}\},\left\{\zeta^{N}\right\}$, for any $\boldsymbol{\eta}=\left(\eta^{N}: N \geq 1\right)$ in $\mathcal{W}$,

$$
\begin{aligned}
\mathbf{E}_{\eta^{N}}\left[T_{\boldsymbol{\zeta}}(\mathcal{W})\right] & \leq \mathbf{E}_{\eta^{N}}\left[T_{\boldsymbol{\xi}}(\mathcal{W})\right]+\mathbf{E}_{\xi^{N}}\left[T_{\boldsymbol{\zeta}}(\mathcal{W})\right] \\
& \leq \frac{\mu_{N}(\mathcal{W})}{\operatorname{cap}_{N}(\boldsymbol{\eta}, \boldsymbol{\xi})} \mathbf{1}\left\{\eta^{N} \neq \xi^{N}\right\}+\frac{\mu_{N}(\mathcal{W})}{\operatorname{cap}_{N}(\boldsymbol{\zeta}, \boldsymbol{\xi})} \mathbf{1}\left\{\zeta^{N} \neq \xi^{N}\right\} \\
& \leq \frac{2 \mu_{N}(\mathcal{W})}{\operatorname{cap}_{N}(\boldsymbol{\xi})} .
\end{aligned}
$$

From this estimate, identity (2.14) and hypothesis (2.15), it follows that

$$
\lim _{N \rightarrow \infty} r_{N}\left(\mathcal{W}, \mathcal{B}^{c}\right) \mathbf{E}_{\eta^{N}}\left[T_{\boldsymbol{\zeta}}(\mathcal{W})\right]=0
$$

which, by (5.1) in Proposition 5.1, implies that

$$
\lim _{N \rightarrow \infty} \frac{\mathbf{E}_{\eta^{N}}\left[T_{\boldsymbol{\zeta}}(\mathcal{W})\right]}{\mathbf{E}_{\eta^{N}}\left[T_{\mathcal{B}^{c}}(\mathcal{W})\right]}=0
$$

This limit corresponds to item (i) of Proposition 4.4 with the point $\boldsymbol{\zeta}$ instead of $\boldsymbol{\xi}$. Item (ii) of Proposition 4.4 follows from the last two assertions of Proposition [5.1. From items (i) and (ii) we conclude that $(\mathcal{W}, \mathcal{W}, \boldsymbol{\zeta})$ is a valley for the trace process $\left\{\eta_{t}^{\mathcal{E}_{N}}: t \geq 0\right\}$. Hence,

$$
\lim _{N \rightarrow \infty} \mathbf{P}_{\eta^{N}}\left[T_{\boldsymbol{\zeta}}(\mathcal{E})<T_{\mathcal{B}^{c}}(\mathcal{E})\right]=1,
$$

which implies condition (V1) for the triple $\left(\mathcal{W}, \mathcal{B}^{c}, \boldsymbol{\zeta}\right)$ because $\left\{T_{\boldsymbol{\zeta}}(\mathcal{E})<T_{\mathcal{B}^{c}}(\mathcal{E})\right\} \subseteq$ $\left\{T_{\zeta}<T_{\mathcal{B}^{c}}\right\}$, proving (5.25). 
By Proposition 6.10 with $A=\{\eta\}, B=\mathcal{B}^{c}$ and $g=\mathbf{1}\{\mathcal{W}\}$, and by identity (2.14), the limit (5.1) can be re-written as

$$
\lim _{N \rightarrow \infty} \frac{\left\langle\mathbf{1}\left\{W_{N}\right\}, f_{N}\left(\boldsymbol{\eta}, \mathcal{B}^{c}\right)\right\rangle_{\mu_{N}} \operatorname{cap}_{N}\left(\mathcal{W}, \mathcal{B}^{c}\right)}{\mu_{N}(\mathcal{W}) \operatorname{cap}_{N}\left(\boldsymbol{\eta}, \mathcal{B}^{c}\right)}=1 .
$$

Replace $\boldsymbol{\eta}$ by $\boldsymbol{\zeta}$ in this formula. By (5.25), the infimum of $f_{N}\left(\boldsymbol{\zeta}, \mathcal{B}^{c}\right)$ over $W_{N}$ converges to 1 as $N \uparrow \infty$. Therefore, (5.24) follows from this observation and the previous identity.

We are now in a position to prove Theorem 2.9. We first show that $(\mathcal{W}, \mathcal{B}, \boldsymbol{\xi})$ is a valley of depth $\theta_{N}=r_{N}\left(\mathcal{W}, \mathcal{B}^{c}\right)^{-1}=\mu_{N}(\mathcal{W}) / \operatorname{cap}_{N}\left(\mathcal{W}, \mathcal{B}^{c}\right)$. Identity (2.14) and Proposition 6.10 show that

$$
r_{N}\left(\mathcal{W}, \mathcal{B}^{c}\right) \mathbf{E}_{\eta^{N}}\left[T_{\mathcal{B}^{c}}(\boldsymbol{\Delta})\right]=\frac{\left\langle\mathbf{1}\left\{\Delta_{N}\right\}, f_{N}\left(\boldsymbol{\eta}, \mathcal{B}^{c}\right)\right\rangle_{\mu_{N}} \operatorname{cap}_{N}\left(\mathcal{W}, \mathcal{B}^{c}\right)}{\mu_{N}(\mathcal{W}) \operatorname{cap}_{N}\left(\boldsymbol{\eta}, \mathcal{B}^{c}\right)}
$$

By Proposition 5.11, (5.24) holds. Since $f_{N}\left(\boldsymbol{\eta}, \mathcal{B}^{c}\right)$ is bounded by one, (5.24) along with hypothesis (2.16) proves (2.11). Since (2.9) and (2.10) follow from (2.15), all the hypotheses of Theorem 2.7 are fulfilled. Therefore, $(\mathcal{W}, \mathcal{B}, \boldsymbol{\xi})$ is a valley of depth $\theta_{N}=r_{N}\left(\mathcal{W}, \mathcal{B}^{c}\right)^{-1}=\mu_{N}(\mathcal{W}) / \operatorname{cap}_{N}\left(\mathcal{W}, \mathcal{B}^{c}\right)$. Last identity follows from Lemma 6.7

Fix now a point $\boldsymbol{\zeta}$ in $\mathcal{W}$. To prove that $(\mathcal{W}, \mathcal{B}, \boldsymbol{\zeta})$ is a valley, we check conditions (i)-(iii) of Proposition 4.4. Property (i) has been proved in (5.26). Since $(\mathcal{W}, \mathcal{B}, \boldsymbol{\xi})$ is a valley, conditions (ii) and (iii) are in force due to the first part of Proposition 4.4. Hence, by the second part of this proposition, $(\mathcal{W}, \mathcal{B}, \boldsymbol{\zeta})$ is a valley of depth $\mathbf{E}_{\zeta^{N}}\left[T_{\mathcal{B}^{c}}(\mathcal{W})\right]$. Finally, since $(\mathcal{W}, \mathcal{B}, \boldsymbol{\xi})$ is a valley, by the first part of this proposition, $\theta_{N}$ and $\mathbf{E}_{\zeta^{N}}\left[T_{\mathcal{B}^{c}}(\mathcal{W})\right]$ are asymptotically equivalent sequences.

Proof of Theorem 2.10. We need to check that all assumptions of Theorem 2.8 are satisfied. As in the proof of Theorem 2.9, conditions (C2), (C3) follow from assumption (H1). It remains to show that $(\mathbf{C 1})$ is fulfilled for all non-absorbing states. Fix such a state $x$. It is enough to prove that

$$
\limsup _{N \rightarrow \infty} \sup _{\eta \in \varepsilon_{N}^{x}} \frac{1}{\theta_{N}} \mathbf{E}_{\eta}\left[T_{\breve{\varepsilon}^{x}}(\boldsymbol{\Delta})\right]=0 .
$$

By Proposition 6.10 and since $f_{\eta_{\mathscr{\varepsilon}^{x}}}$ is bounded by 1 , the expectation is less than or equal to $\mu_{N}(\boldsymbol{\Delta}) / \operatorname{cap}\left(\eta, \breve{\mathcal{E}}^{x}\right)$. By (5.24), we may replace asymptotically $\boldsymbol{\eta}$ by $\mathcal{E}^{x}$ in the previous capacity. By Lemma 6.7] $\operatorname{cap}\left(\mathcal{E}^{x}, \breve{\mathcal{E}}^{x}\right)$ is equal to $\mu_{N}\left(\mathcal{E}^{x}\right) r_{N}\left(\mathcal{E}^{x}, \breve{\mathcal{E}}^{x}\right)$. In conclusion, we have shown that

$$
\limsup _{N \rightarrow \infty} \sup _{\eta \in \varepsilon_{N}^{x}} \frac{1}{\theta_{N}} \mathbf{E}_{\eta}\left[T_{\breve{\varepsilon}^{x}}(\boldsymbol{\Delta})\right] \leq \limsup _{N \rightarrow \infty} \frac{1}{\theta_{N} r_{N}\left(\mathcal{E}^{x}, \breve{\mathcal{E}}^{x}\right)} \frac{\mu_{N}(\boldsymbol{\Delta})}{\mu_{N}\left(\mathcal{E}^{x}\right)} .
$$

Since $x$ is a non-absorbing point, by assumptions (H0), (H2), the right hand side is equal to 0 . This concludes the proof.

Proof of Remark 2.11. We need to show that (H2) holds for non-absorbing states and that (M3) holds for absorbing states. Clearly, (H2) follows from (H2') for non-absorbing states. On the other hand, by Proposition 5.7, (M2) is fulfilled. Hence, by Lemma 4.7. (M3) for absorbing (and non-absorbing) states is a consequence of (5.27). By (5.28), assumption (H2') implies (5.27), which concludes the proof. 


\section{Continuous time Markov chains}

We state in this section several properties of continuous time Markov chains used throughout the article. We start assuming that the holding rates are strictly positive and finite and that the jump chain associated is irreducible and recurrent. We add assumptions as we progress. At the end, we consider the case of positive recurrent, reversible Markov chains whose holding times belong to $L^{1}(\mu)$, where $\mu$ is the unique invariant probability measure.

Consider a countable set $E$ and a matrix $R: E \times E \rightarrow \mathbb{R}$ such that $R(\eta, \xi) \geq 0$, $\eta \neq \xi,-\infty<R(\eta, \eta)<0, \sum_{\xi \neq \eta} R(\eta, \xi)=0, \eta \in E$. Let $\lambda(\eta)=-R(\eta, \eta)$. Since $\lambda(\eta)$ is finite and strictly positive, we may define the transition probabilities $\{p(\eta, \xi): \eta, \xi \in E\}$ as

$$
p(\eta, \xi)=\frac{1}{\lambda(\eta)} R(\eta, \xi) \text { for } \eta \neq \xi,
$$

and $p(\eta, \eta)=0$ for $\eta \in E$. We assume throughout this section that $\{p(\eta, \xi)$ : $\eta, \xi \in E\}$ are the transition probabilities of a irreducible and recurrent discrete time Markov chain.

We claim that there exists a unique stochastic semigroup $\left\{p_{t}: t \geq 0\right\}$ on $E$ satisfying

$$
\lim _{t \downarrow 0} \frac{p_{t}(\eta, \xi)-p_{0}(\eta, \xi)}{t}=R(\eta, \xi) \quad \text { and } \quad p_{0}(\eta, \xi)=\delta_{\eta, \xi}
$$

for every $\eta, \xi \in E$, where $\delta_{\eta, \xi}$ is the delta of Kroenecker. To prove the existence, we construct a Markov process $\left\{\eta_{t}: t \geq 0\right\}$ on $E$ whose Markov semigroup satisfies (6.2). We shall use this construction in some of the proofs below.

Let $Y=\left\{Y_{n}: n \geq 0\right\}$ be an irreducible, recurrent, $E$-valued discrete time Markov chain with transition probabilities $\{p(\eta, \xi): \eta, \xi \in E\}$ given by (6.1). Let $\left(e_{n}: n \geq 0\right)$ be a sequence of i.i.d. mean one exponential random variables, independent of $Y$. We associate to every sample path of $Y$ the sequence of random times $T=\left(T_{n}: n \geq 0\right)$ given by

$$
T_{n}=\frac{e_{n}}{\lambda\left(Y_{n}\right)} .
$$

Since $Y$ is recurrent, $\sum_{i \geq 0} T_{i}=\infty$ a.s. In particular, the time-change

$$
\alpha(t)=\min \left\{n \geq 0: \sum_{i=0}^{n} T_{i}>t\right\}
$$

is a.s. finite for every $t \geq 0$ and $\eta_{t}=Y_{\alpha(t)}$ is a.s. well defined for all $t \geq 0$. In Theorem 2.8.1 of [17] it is proved that $\left\{\eta_{t}: t \geq 0\right\}$ is a strong Markov process with respect to the filtration $\left\{\mathcal{F}_{t}: t \geq 0\right\}, \mathcal{F}_{t}=\sigma\left(\eta_{s}: s \leq t\right)$. The stochastic semigroup corresponding to $\left\{\eta_{t}: t \geq 0\right\}$ fulfills (6.2), as follows from the proof of Theorem 2.8.4 in [17. On the other hand, the uniqueness of the stochastic semigroup is a consequence of Theorem (51) in Chapter 7 of [11] along with the recurrence of the transition probabilities $p(\cdot, \cdot)$. Note that there is no explosion since $\sum_{i \geq 0} T_{i}=\infty$ a.s.

In conclusion, a collection of nonnegative numbers $\{R(\eta, \xi): \eta, \xi \in E\}$ satisfying the conditions listed at the beginning of this section determines uniquely the law of a strong Markov process $\left\{\eta_{t}: t \geq 0\right\}$. We shall refer to $R(\cdot, \cdot), \lambda(\cdot)$ and $p(\cdot, \cdot)$ as the transition rates, holding rates and jump probabilities of $\left\{\eta_{t}: t \geq 0\right\}$, respectively. 
The Markov chain $Y=\left\{Y_{n}: n \geq 0\right\}$ is called the jump chain associated to $\left\{\eta_{t}\right.$ : $t \geq 0\}$.

Of course, since the jump chain $Y$ is irreducible and recurrent, so is the corresponding Markov process $\left\{\eta_{t}: t \geq 0\right\}$. In consequence, $\left\{\eta_{t}: t \geq 0\right\}$ has an invariant measure $\mu$ which is unique up to scalar multiples. Moreover,

$$
M(\eta):=\lambda(\eta) \mu(\eta), \quad \eta \in E,
$$

is the invariant measure for the jump chain $Y$, also unique up to scalar multiples. The proofs of these assertions can be found in Sections 3.4 and 3.5 of [17.

Recall that $\tau_{A}: D\left(\mathbb{R}_{+}, E\right) \rightarrow \mathbb{R}_{+}, A \subseteq E$, denotes the hitting time of the set $A$ :

$$
\tau_{A}(e .)=\inf \left\{t>0: e_{t} \in A\right\} .
$$

Let $T_{A}:=\tau_{A}(\eta$. $)$ and $T_{\eta}:=T_{\{\eta\}}, \eta \in E$. Define the stopping time $\tau_{A}^{+}: D\left(\mathbb{R}_{+}, E\right) \rightarrow$ $\mathbb{R}_{+}$as the first return to $A$ :

$$
\tau_{A}^{+}(e .)=\inf \left\{t>0: e_{t} \in A, e_{s} \neq e_{0} \text { for some } 0<s<t\right\},
$$

and let $T_{A}^{+}:=\tau_{A}^{+}(\eta),. T_{\eta}^{+}:=T_{\{\eta\}}^{+}, \eta \in E$.

Let $\mathbf{P}_{\eta}, \eta \in E$, be the probability measure under which the jump chain $\left\{Y_{n}\right.$ : $n \geq 0\}$ and the Markov chain $\left\{\eta_{t}: t \geq 0\right\}$ start from $\eta$. Expectation with respect to $\mathbf{P}_{\eta}$ is denoted by $\mathbf{E}_{\eta}$. It follows from the proof of Theorem 3.5.3 in [17] that for any $\eta \in E$

$$
\mu(\xi)=\mathbf{E}_{\eta}\left[\int_{0}^{T_{\eta}^{+}} \mathbf{1}\left\{\eta_{s}=\xi\right\} d s\right], \quad \xi \in E,
$$

is an invariant measure for $\left\{\eta_{t}: t \geq 0\right\}$.

6.1. The trace process. We present in this subsection some elementary properties of trace processes and we state some identities used throughout the article.

Let $h: E \rightarrow \mathbb{R}_{+}$be a nonnegative function with nonempty support $F$ :

$$
F:=\{\eta \in E: h(\eta)>0\} \neq \varnothing .
$$

Define the additive functional $\left\{\mathcal{T}_{t}^{h}: t \geq 0\right\}$ as

$$
\mathcal{T}_{t}^{h}:=\int_{0}^{t} h\left(\eta_{s}\right) d s .
$$

Notice that $\mathcal{T}_{t}^{h} \in \mathbb{R}_{+}, \mathbf{P}_{\eta^{-}}$-a.s. for every $\eta \in E$ and $t \geq 0$. Denote by $\left\{\mathcal{S}_{t}^{h}: t \geq 0\right\}$ the generalized inverse of $\mathcal{T}_{t}^{h}$ :

$$
\mathcal{S}_{t}^{h}:=\sup \left\{s \geq 0: \mathcal{T}_{s}^{h} \leq t\right\} .
$$

Since $\left\{\eta_{t}: t \geq 0\right\}$ is irreducible and recurrent, $\lim _{t \rightarrow \infty} \mathcal{T}_{t}^{h}=\infty, \mathbf{P}_{\eta^{-}}$a.s. for every $\eta \in E$. Therefore, the random path $\left\{\eta_{t}^{h}: t \geq 0\right\}$, given by $\eta_{t}^{h}=\eta_{\mathcal{S}_{t}^{h}}$, is $\mathbf{P}_{\eta^{-}}$-a.s. well defined for all $\eta \in E$ and takes value in the set $F$. We call the process $\left\{\eta_{t}^{h}: t \geq 0\right\}$ the $h$-trace of $\left\{\eta_{t}: t \geq 0\right\}$. Clearly, $\left\{\eta_{t}^{h}: t \geq 0\right\}$ coincides with the trace of $\left\{\eta_{t}: t \geq 0\right\}$ on $F$, defined in Section 2, if $h=\mathbf{1}\{F\}$.

A change of variables shows that for any subset $B$ of $F$ and for any function $f: F \rightarrow \mathbb{R}_{+}$,

$$
\int_{0}^{\tau_{B}\left(\eta^{h}\right)} f\left(\eta_{t}^{h}\right) d t=\int_{0}^{T_{B}} f\left(\eta_{t}\right) h\left(\eta_{t}\right) d t
$$


$\mathbf{P}_{\eta^{-}}$a.s. for every $\eta \in E$. This identity also holds if we replace $\tau_{B}\left(\eta_{.}^{h}\right), T_{B}$ by $\tau_{B}^{+}\left(\eta^{h}\right), T_{B}^{+}$, respectively. Furthermore, for any two disjoint subsets $A, B$ of $F$, it follows from the construction of the Markov chain $\left\{\eta_{t}^{h}: t \geq 0\right\}$ that

$$
\mathbf{P}_{\eta}\left[\tau_{A}\left(\eta_{.}^{h}\right)<\tau_{B}\left(\eta_{.}^{h}\right)\right]=\mathbf{P}_{\eta}\left[T_{A}<T_{B}\right]
$$

for all $\eta$ in $F$. This identity needs to be reformulated if we replace the hitting times by return times. Indeed, if the process starting from $\eta$ returns to $F$ by $\eta$, while in the original version the process returned to $\eta$, in the trace version the process never left $\eta$. We claim that for all $\eta \in F$ and all disjoint subsets $A, B$ of $F$,

$$
\mathbf{P}_{\eta}\left[\tau_{A}^{+}\left(\eta^{h}\right)<\tau_{B}^{+}\left(\eta_{.}^{h}\right)\right]=\mathbf{P}_{\eta}\left[T_{A}^{+}<T_{B}^{+} \mid T_{F}^{+}=T_{F \backslash\{\eta\}}\right] .
$$

To derive this identity, intersect the event $\left\{\tau_{A}^{+}\left(\eta^{h}\right)<\tau_{B}^{+}\left(\eta^{h}\right)\right\}$ with the partition $\left\{T_{F}^{+}=T_{F \backslash\{\eta\}}\right\},\left\{T_{F}^{+}=T_{\eta}^{+}\right\}$and apply the strong Markov property to the second piece to get that

$$
\begin{aligned}
\mathbf{P}_{\eta}\left[\tau_{A}^{+}\left(\eta_{.}{ }^{h}\right)<\tau_{B}^{+}\left(\eta_{.}{ }^{h}\right)\right]=\mathbf{P}_{\eta}\left[\tau_{A}^{+}\left(\eta_{.}^{h}\right)\right. & \left.<\tau_{B}^{+}\left(\eta_{.}^{h}\right) ; T_{F}^{+}=T_{F \backslash\{\eta\}}\right] \\
& +\mathbf{P}_{\eta}\left[T_{F}^{+}=T_{\eta}^{+}\right] \mathbf{P}_{\eta}\left[\tau_{A}^{+}\left(\eta_{.}{ }^{h}\right)<\tau_{B}^{+}\left(\eta_{.}{ }^{h}\right)\right] .
\end{aligned}
$$

To conclude, observe that on the set $\left\{T_{F}^{+}=T_{F \backslash\{\eta\}}\right\}$ we may replace $\eta^{h}$. by $\eta$. in the event $\left\{\tau_{A}^{+}\left(\eta^{h}\right)<\tau_{B}^{+}\left(\eta^{h}\right)\right\}$.

Proposition 6.1. Under $\left\{\mathbf{P}_{\eta}: \eta \in F\right\},\left\{\eta_{t}^{h}: t \geq 0\right\}$ is an irreducible, recurrent, strong Markov chain with transition rates given by

$$
R^{h}(\eta, \xi)=\frac{\lambda(\eta)}{h(\eta)} \mathbf{P}_{\eta}\left[T_{F}^{+}=T_{\xi}^{+}\right], \quad \eta, \xi \in F, \eta \neq \xi
$$

Proof. Recall the explicit construction of the Markov chain $\left\{\eta_{t}: t \geq 0\right\}$ presented in the previous subsection. To derive the $h$-trace from this construction, we consider first the trace of the jump chain $\left\{Y_{n}: n \geq 0\right\}$ on $F$.

Define the sequence of times $\left\{t_{n}: n \geq 0\right\}$ as $t_{0}=0, t_{1}=\inf \left\{n \geq 1: Y_{n} \in F\right\}$ and $t_{n+1}=t_{n}+t_{1} \circ \Theta_{t_{n}}, n \geq 1$, where $\left\{\Theta_{k}: k \geq 1\right\}$ are the discrete time shift operators. Let $Y^{\mathfrak{h}}=\left\{Y_{n}^{\mathfrak{h}}: n \geq 0\right\}$ be given by $Y_{n}^{\mathfrak{h}}=Y_{t_{n}}$. When the jump chain $\left\{Y_{n}: n \geq 0\right\}$ starts in $F, Y^{\mathfrak{h}}=\left\{Y_{n}^{\mathfrak{h}}: n \geq 0\right\}$ defines a $F$-valued discrete time Markov chain with transition probabilities

$$
\mathfrak{p}(\eta, \xi)=\mathbf{P}_{\eta}\left[T_{F}^{+}=T_{\xi}^{+}\right], \quad \eta, \xi \in F .
$$

Note that $\mathfrak{p}(\eta, \eta)$ may be strictly positive and that $Y^{\mathfrak{h}}$ inherits the irreducibility and the recurrence properties from $Y$.

Let $T^{\mathfrak{h}}=\left\{T_{n}^{\mathfrak{h}}: n \geq 0\right\}$ be the sequence

$$
T_{n}^{\mathfrak{h}}=h\left(Y_{n}^{\mathfrak{h}}\right) \frac{e_{t_{n}}}{\lambda\left(Y_{n}^{\mathfrak{h}}\right)}, \quad n \geq 0 .
$$

By definition, the $h$-trace of $\left\{\eta_{t}: t \geq 0\right\}$ is given by $\eta_{t}^{h}=Y_{\alpha(t)}^{\mathfrak{h}}, t \geq 0$, where $\alpha(\cdot)$ represents the time-change (6.3) with $Y^{\mathfrak{h}}$ and $T^{\mathfrak{h}}$ in place of $Y$ and $T$, respectively. Note that $\left\{e_{t_{n}}: n \geq 0\right\}$ is a sequence of i.i.d. mean one exponential random variables independent of the process $\left\{Y_{n}^{\mathfrak{h}}: n \geq 0\right\}$. By this observation and by the proof of Theorem 2.8.1 in [17, $\left\{\eta_{t}^{h}: t \geq 0\right\}$ is a strong Markov process on $F$. 
The irreducibility and the recurrence of $\left\{\eta_{t}^{h}: t \geq 0\right\}$ are inherited from the process $Y^{\mathfrak{h}}$. On the other hand, the transition rates $\left\{R^{h}(\eta, \xi): \eta, \xi \in F\right\}$ of the strong Markov process $\left\{\eta_{t}^{h}: t \geq 0\right\}$ are given by

$$
R^{h}(\eta, \xi):=\lim _{t \downarrow 0} \frac{\mathbf{P}_{\eta}\left[\eta_{t}^{h}=\xi\right]}{t}=\frac{\mathfrak{p}(\eta, \xi)}{\mathbf{E}_{\eta}\left[T_{0}^{\mathfrak{h}}\right]}=\frac{\lambda(\eta)}{h(\eta)} \mathbf{P}_{\eta}\left[T_{F}^{+}=T_{\xi}^{+}\right]
$$

for $\eta, \xi \in F, \eta \neq \xi$. The second identity follows from the proof of Theorem 2.8.4 in [17.

It follows from this proposition that the holding rates $\left\{\lambda^{h}(\eta): \eta \in F\right\}$ and the jump probabilities $\left\{p^{h}(\eta, \xi): \eta, \xi \in F\right\}$ of the $h$-trace process $\left\{\eta_{t}^{h}: t \geq 0\right\}$ are given by

$$
\lambda^{h}(\eta)=\frac{\lambda(\eta)}{h(\eta)} \mathbf{P}_{\eta}\left[T_{F}^{+}=T_{F \backslash\{\eta\}}^{+}\right],
$$

and, for $\eta \neq \xi$,

$$
p^{h}(\eta, \xi)=\frac{\mathbf{P}_{\eta}\left[T_{F}^{+}=T_{\xi}^{+}\right]}{\mathbf{P}_{\eta}\left[T_{F}^{+}=T_{F \backslash\{\eta\}}^{+}\right]}=\mathbf{P}_{\eta}\left[T_{F \backslash\{\eta\}}=T_{\xi}\right] .
$$

Note that $p^{h}(\cdot, \cdot)$ depends on $h$ only through its support. The second identity is obtained by intersecting the event $\left\{T_{F \backslash\{\eta\}}=T_{\xi}\right\}$ with the partition $\left\{T_{F}^{+}=T_{F \backslash\{\eta\}}\right\}$, $\left\{T_{F}^{+}=T_{\eta}^{+}\right\}$and applying the strong Markov property to the second piece as in the proof of (6.8).

When $h$ is the indicator function of a set $F$, we obtain an explicit formula for the transition rates of the trace process.

Corollary 6.2. Let $R^{F}$ stand for the transition rates of $\left\{\eta_{t}^{h}: t \geq 0\right\}$ when $h=$ $\mathbf{1}\{F\}$. Then, for $\eta, \xi$ in $F, \eta \neq \xi$,

$$
R^{F}(\eta, \xi)=R(\eta, \xi)+\sum_{\zeta \in F^{c}} R(\eta, \zeta) \mathbf{P}_{\zeta}\left[T_{F}=T_{\xi}\right]
$$

Proof. By Proposition 6.1 $R^{F}(\eta, \xi)=\lambda(\eta) \mathbf{P}_{\eta}\left[T_{F}^{+}=T_{\xi}^{+}\right]$. Consider the stopping time $T_{F^{c}}^{+}$with the convention that $T_{F^{c}}^{+}=\infty$ if $F^{c}=\varnothing$. Decomposing the event $\left\{T_{F}^{+}=T_{\xi}^{+}\right\}$according to the event $\left\{T_{F}^{+}<T_{F^{c}}^{+}\right\}$and its complement, we get

$$
R^{F}(\eta, \xi)=\lambda(\eta) \mathbf{P}_{\eta}\left[T_{F}^{+}=T_{\xi}^{+} ; T_{F}^{+}<T_{F^{c}}^{+}\right]+\lambda(\eta) \mathbf{P}_{\eta}\left[T_{F}^{+}=T_{\xi}^{+} ; T_{F^{c}}^{+}<T_{F}^{+}\right] .
$$

The first probability on the right hand side is equal to $\mathbf{P}_{\eta}\left[T_{E}^{+}=T_{\xi}^{+}\right]=p(\eta, \xi)$, while the second term, by the strong Markov property, is equal to

$$
\sum_{\zeta \in F^{c}} R(\eta, \zeta) \mathbf{P}_{\zeta}\left[T_{F}=T_{\xi}\right]
$$

This concludes the proof of the corollary.

The previous corollary provides an explicit formula for the rates $R^{F}$ in terms of the holding times $\lambda$ and the transition probabilities $p$ in the case where $F=E \backslash\left\{\xi_{0}\right\}$ :

$$
R^{F}(\eta, \xi)=R(\eta, \xi)+R\left(\eta, \xi_{0}\right) p\left(\xi_{0}, \xi\right)
$$

for $\eta \neq \xi,\{\eta, \xi\} \subseteq E \backslash\left\{\xi_{0}\right\}$. In particular, if $E$ is a finite set, the rates $R^{F}$ can be obtained recursively. 
Since $\left\{\eta_{t}^{h}: t \geq 0\right\}$ is recurrent and irreducible, it has an invariant measure which is unique up to scalar multiplies. Let $\mu$ be an invariant measure for $\left\{\eta_{t}: t \geq 0\right\}$ and denote by $\mu_{o}^{h}$ the measure on $F$ given by

$$
\mu_{o}^{h}(\xi):=h(\xi) \mu(\xi), \quad \xi \in F
$$

Proposition 6.3. $\mu_{o}^{h}$ is an invariant measure for $\left\{\eta_{t}^{h}: t \geq 0\right\}$. In particular, if $h$ is $\mu$-integrable then $\left\{\eta_{t}^{h}: t \geq 0\right\}$ is positive recurrent. Moreover, if $\mu$ is a reversible measure for $\left\{\eta_{t}: t \geq 0\right\}$ then $\mu_{o}^{h}$ is a reversible measure for $\left\{\eta_{t}^{h}: t \geq 0\right\}$.

Proof. Without loss of generality, we may suppose that $\mu$ is of the form (6.5) for some $\eta \in F$. Thus, by (6.7), for any $\xi \in E$,

$$
h(\xi) \mu(\xi)=\mathbf{E}_{\eta}\left[\int_{0}^{T_{\eta}^{+}} h\left(\eta_{s}\right) \mathbf{1}\left\{\eta_{s}=\xi\right\} d s\right]=\mathbf{E}_{\eta}\left[\int_{0}^{\tau_{\eta}^{+}\left(\eta^{h}\right)} \mathbf{1}\left\{\eta_{s}^{h}=\xi\right\} d s\right] .
$$

This shows that $\mu_{o}^{h}$ is an invariant measure for the $h$-trace process. The second assertion follows from Theorem 3.5.3 in [17].

Suppose now that $\mu$ is reversible for $R(\cdot, \cdot)$. Then, the measure $M$ defined in (6.4) is a reversible measure for the jump chain $Y=\left\{Y_{n}: n \geq 0\right\}$. Since the events $\left\{T_{F}^{+}=T_{\xi}^{+}\right\}$and $\left\{T_{F}^{+}=T_{\eta}^{+}\right\}$depend only on $Y$,

$$
M(\eta) \mathbf{P}_{\eta}\left[T_{F}^{+}=T_{\xi}^{+}\right]=M(\xi) \mathbf{P}_{\xi}\left[T_{F}^{+}=T_{\eta}^{+}\right]
$$

for any $\eta, \xi \in F, \eta \neq \xi$. In consequence, by the formula for $R^{h}(\cdot, \cdot)$ obtained in Proposition 6.1 $\mu_{o}^{h}$ is reversible for the $h$-trace process.

6.2. Positive recurrent case. We assume from now on that the Markov chain $\left\{\eta_{t}: t \geq 0\right\}$ is positive recurrent. Denote by $\mu$ its unique invariant probability measure.

Replacement Lemma. For any probability measure $\nu$ on $E$, we denote by $\langle\cdot\rangle_{\nu}$ the expected value with respect to $\nu$.

Lemma 6.4. Fix a function $g: E \rightarrow \mathbb{R}$ with nonempty support, integrable with respect to $\mu$ and such that $\langle g\rangle_{\mu}=0$. Fix also some $\xi$ in $A=\{\eta: g(\eta) \neq 0\}$. For every $t>0$,

$$
\sup _{\eta \in E}\left|\mathbf{E}_{\eta}\left[\int_{0}^{t} g\left(\eta_{s}\right) d s\right]\right| \leq 2 \sup _{\eta \in A} \mathbf{E}_{\eta}\left[\int_{0}^{T_{\xi}}\left|g\left(\eta_{s}\right)\right| d s\right] .
$$

Proof. Let $\left\{\Theta_{t}: t \geq 0\right\}$ stand for the time shift operators on $D\left(\mathbb{R}_{+}, E\right)$. Define the random times $H_{0}=0, H_{1}=T_{\xi}^{+}$and $H_{j+1}=H_{j}+\tau_{\xi}^{+} \circ \Theta_{H_{j}}(\eta$.), $j \geq 1$. Fix an arbitrary $\eta \in E$ and let $h: E \rightarrow \mathbb{R}_{+}$be a nonnegative function, integrable with respect to $\mu$. By Proposition 6.3, the trace process $\left\{\eta_{t}^{h}: t \geq 0\right\}$ is positive recurrent so that

$$
\mathbf{E}_{\eta}\left[\int_{0}^{T_{\xi}} h\left(\eta_{s}\right) d s\right]=\mathbf{E}_{\eta}\left[\tau_{\xi}\left(\eta_{.}^{h}\right)\right]<\infty
$$


Write

$$
\begin{aligned}
\mathbf{E}_{\eta}\left[\int_{0}^{t} h\left(\eta_{s}\right) d s\right] & =\sum_{j \geq 0} \mathbf{E}_{\eta}\left[\int_{0}^{t} h\left(\eta_{s}\right) d s \mathbf{1}\left\{H_{j} \leq t<H_{j+1}\right\}\right] \\
& =\sum_{j \geq 0} \mathbf{E}_{\eta}\left[\int_{0}^{H_{j+1}} h\left(\eta_{s}\right) d s \mathbf{1}\left\{H_{j} \leq t<H_{j+1}\right\}\right] \\
& -\sum_{j \geq 0} \mathbf{E}_{\eta}\left[\int_{t}^{H_{j+1}} h\left(\eta_{s}\right) d s \mathbf{1}\left\{H_{j} \leq t<H_{j+1}\right\}\right] .
\end{aligned}
$$

In the last equation, we used the fact that both terms on the right hand side are finite. To prove it, notice first that the second term is bounded by the first one. By Tonelli's theorem, the first term is equal to

$$
\begin{gathered}
\sum_{j \geq 0} \sum_{k=0}^{j} \mathbf{E}_{\eta}\left[\int_{H_{k}}^{H_{k+1}} h\left(\eta_{s}\right) d s \mathbf{1}\left\{H_{j} \leq t<H_{j+1}\right\}\right] \\
=\sum_{k \geq 0} \mathbf{E}_{\eta}\left[\int_{H_{k}}^{H_{k+1}} h\left(\eta_{s}\right) d s \mathbf{1}\left\{H_{k} \leq t\right\}\right] .
\end{gathered}
$$

Taking conditional expectation with respect to $\mathcal{F}_{H_{k}}$, by the strong Markov property, this sum is equal to

$$
\mathbf{E}_{\eta}\left[\int_{0}^{H_{1}} h\left(\eta_{s}\right) d s\right]+\mathbf{E}_{\xi}\left[\int_{0}^{H_{1}} h\left(\eta_{s}\right) d s\right] \sum_{k \geq 1} \mathbf{P}_{\eta}\left[H_{k} \leq t\right]
$$

The first term of this sum is finite by (6.10). In the second expectation, $\xi$ appears instead of $\eta$, and the expectation is equal to $\langle h\rangle_{\mu}$ by (6.5). Finally, the sum is finite by the strong Markov property and because $\mathbf{P}_{\xi}\left[T_{\xi}^{+} \leq t\right]$ is strictly smaller than 1 .

To estimate the last term in (6.11), note that the event $\left\{H_{j} \leq t<H_{j+1}\right\}$ belongs to $\mathcal{F}_{t}$ and that on this set $H_{j+1}=t+\tau_{\xi}^{+} \circ \Theta_{t}(\eta$.). Therefore, by the Markov property,

$$
\sum_{j \geq 0} \mathbf{E}_{\eta}\left[\int_{t}^{H_{j+1}} h\left(\eta_{s}\right) d s \mathbf{1}\left\{H_{j} \leq t<H_{j+1}\right\}\right]=\mathbf{E}_{\eta}\left[\mathbf{E}_{\eta_{t}}\left[\int_{0}^{H_{1}} h\left(\eta_{s}\right) d s\right]\right] .
$$

Putting together the previous identities, we get that the left hand side of (6.11) is equal to

$$
\mathbf{E}_{\eta}\left[\int_{0}^{H_{1}} h\left(\eta_{s}\right) d s\right]+\langle h\rangle_{\mu} \sum_{k \geq 1} \mathbf{P}_{\eta}\left[H_{k} \leq t\right]-\mathbf{E}_{\eta}\left[\mathbf{E}_{\eta_{t}}\left[\int_{0}^{H_{1}} h\left(\eta_{s}\right) d s\right]\right] .
$$

Applying the previous identity to $g^{+}$and $g^{-}$, since $\langle g\rangle_{\mu}=0$, we obtain that

$$
\mathbf{E}_{\eta}\left[\int_{0}^{t} g\left(\eta_{s}\right) d s\right]=\mathbf{E}_{\eta}\left[\int_{0}^{H_{1}} g\left(\eta_{s}\right) d s\right]-\mathbf{E}_{\eta}\left[\mathbf{E}_{\eta_{t}}\left[\int_{0}^{H_{1}} g\left(\eta_{s}\right) d s\right]\right] .
$$

We claim that we may replace the stopping time $H_{1}$ by $T_{\xi}$ in both terms of the

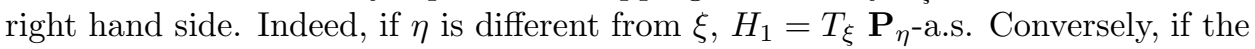
starting point $\eta$ is equal to $\xi, T_{\xi}=0$ so that, by (6.5),

$$
\mathbf{E}_{\eta}\left[\int_{0}^{H_{1}} g\left(\eta_{s}\right) d s\right]=0=\mathbf{E}_{\eta}\left[\int_{0}^{T_{\xi}} g\left(\eta_{s}\right) d s\right]
$$


Thus, taking the supremum over $E$, we have proved that

$$
\sup _{\eta \in E}\left|\mathbf{E}_{\eta}\left[\int_{0}^{t} g\left(\eta_{s}\right) d s\right]\right| \leq 2 \sup _{\eta \in E}\left|\mathbf{E}_{\eta}\left[\int_{0}^{T_{\xi}} g\left(\eta_{s}\right) d s\right]\right| .
$$

Finally, since $g$ vanishes outside $A$ and since $\xi$ belongs to $A$, by the strong Markov property,

$$
\mathbf{E}_{\eta}\left[\int_{0}^{T_{\xi}} g\left(\eta_{s}\right) d s\right]=\mathbf{E}_{\eta}\left[\int_{T_{A}}^{T_{\xi}} g\left(\eta_{s}\right) d s\right]=\mathbf{E}_{\eta}\left[\mathbf{E}_{\eta_{T_{A}}}\left[\int_{0}^{T_{\xi}} g\left(\eta_{s}\right) d s\right]\right] .
$$

Therefore,

$$
\sup _{\eta \in E}\left|\mathbf{E}_{\eta}\left[\int_{0}^{T_{\xi}} g\left(\eta_{s}\right) d s\right]\right| \leq \sup _{\eta \in A}\left|\mathbf{E}_{\eta}\left[\int_{0}^{T_{\xi}} g\left(\eta_{s}\right) d s\right]\right|
$$

This concludes the proof of the lemma.

Let $S$ be a finite set and let $\pi=\left\{A^{x}: x \in S\right\}$ be a partition of $E$. Denote by $\mu^{x}, x \in S$, the stationary measure $\mu$ conditioned on $A^{x}: \mu^{x}(\cdot)=\mu\left(\cdot \mid A^{x}\right)$. Also, for each $\mu$-integrable function $g$ denote by $\langle g \mid \pi\rangle_{\mu}: E \rightarrow \mathbb{R}$ the conditional expectation of $g$, under $\mu$, given the $\sigma$-algebra generated by $\pi$ :

$$
\langle g \mid \pi\rangle_{\mu}=\sum_{x \in S}\langle g\rangle_{\mu^{x}} \mathbf{1}\left\{A^{x}\right\} .
$$

The next result shows that if the process thermalizes quickly in each set of the partition, we may replace time averages of a bounded function by time averages of the conditional expectation. This statement plays a key role in our investigation of metastability. It assumes the existence of an attractor, but similar versions should exist under weaker assumptions on thermalization.

For each $x \in S$ and $\mu$-integrable function $g: E \rightarrow \mathbb{R}$, let

$$
g^{x}:=\left(g-\langle g\rangle_{\mu^{x}}\right) \mathbf{1}\left\{A^{x}\right\}
$$

and fix some state $\xi_{x}$ in $A^{x}$, for each $x$ in $S$. Next statement follows from Lemma 6.4 applied to each $g^{x}, x \in S$. Note that the right hand side does not depend on time.

Corollary 6.5. Let $g: E \rightarrow \mathbb{R}$ be an integrable function. Then,

$$
\sup _{\eta \in E}\left|\mathbf{E}_{\eta}\left[\int_{0}^{t}\left\{g-\langle g \mid \pi\rangle_{\mu}\right\}\left(\eta_{s}\right) d s\right]\right| \leq 2 \sum_{x \in S} \sup _{\eta \in A^{x}} \mathbf{E}_{\eta}\left[\int_{0}^{T_{\xi_{x}}}\left|g^{x}\left(\eta_{s}\right)\right| d s\right] .
$$

Clearly, the right hand side in the previous corollary is bounded above by

$$
4 \sum_{x \in S}\|g\|_{x} \sup _{\eta \in A^{x}} \mathbf{E}_{\eta}\left[\int_{0}^{T_{\xi_{x}}} \mathbf{1}\left\{\eta_{s} \in A^{x}\right\} d s\right]
$$

where $\|g\|_{x}=\sup \left\{|g(\eta)|: \eta \in A^{x}\right\}$. 
Mean set rates. Let $h: E \rightarrow \mathbb{R}_{+}$be a nonnegative function satisfying (6.6) and belonging to $L^{1}(\mu)$. By Propositions 6.1 and 6.3, $\left\{\eta_{t}^{h}: t \geq 0\right\}$ is irreducible and positive recurrent. Moreover, its invariant probability measure, denoted by $\mu^{h}$, is given by

$$
\mu^{h}(\xi)=\frac{h(\xi)}{\langle h\rangle_{\mu}} \mu(\xi), \quad \xi \in F .
$$

For each pair $A, B$ of disjoint subsets of $F$, denote by $r_{h}(A, B)$ the average rate at which the $h$-trace process jumps from $A$ to $B$ :

$$
\begin{aligned}
r_{h}(A, B) & :=\frac{1}{\mu^{h}(A)} \sum_{\eta \in A} \mu^{h}(\eta) \sum_{\xi \in B} R^{h}(\eta, \xi) \\
& =\frac{1}{\langle h, \mathbf{1}\{A\}\rangle_{\mu}} \sum_{\eta \in A} M(\eta) \mathbf{P}_{\eta}\left[T_{F}^{+}=T_{B}^{+}\right],
\end{aligned}
$$

where $M$ has been introduced in (6.4). We used relation 6.12) and Proposition 6.1 in the last equality. We shall refer to $r_{h}(\cdot, \cdot)$ as the mean set rates associated to the trace process.

When $h$ is the indicator function of a set $F$, we denote $r_{h}$ by $r_{F}$. In this case,

$$
\mu(A) r_{F}(A, B)=\sum_{\eta \in A} M(\eta) \mathbf{P}_{\eta}\left[T_{B}^{+}<T_{F \backslash B}^{+}\right]
$$

6.3. The reversible case. From now on, we shall assume in addition that the process is reversible with respect to the invariant probability measure $\mu$ and that the measure $M$ is finite:

$$
\sum_{\eta \in E} M(\eta)=\sum_{\eta \in E} \lambda(\eta) \mu(\eta)<\infty .
$$

In particular, the mean set rates $r_{h}(A, B)$ are finite.

Assumption (6.14) reduces the potential theory of continuous time Markov chains to the potential theory of discrete time Markov chains. Recall from Subsection 2.4 that $\langle\cdot, \cdot\rangle_{M}$ represents the scalar product in $L^{2}(M)$, that $P: L^{2}(M) \rightarrow L^{2}(M)$ stands for the bounded operator defined by $(P f)(\eta)=\sum_{\xi \in E} p(\eta, \xi) f(\xi)$, and that $D(f)=\langle(I-P) f, f\rangle_{M}, f \in L^{2}(M)$, is the Dirichlet form associated to the Markov process $\left\{\eta_{t}: t \geq 0\right\}$. A simple computation shows that for every $f$ in $L^{2}(M)$,

$$
D(f)=\frac{1}{2} \sum_{\eta, \xi \in E} M(\eta) p(\eta, \xi)\{f(\xi)-f(\eta)\}^{2} .
$$

Fix two disjoint subsets $A, B$ of $E$ and recall that $\mathcal{C}(A, B):=\left\{f \in L^{2}(M)\right.$ : $f(\eta)=1 \forall \eta \in A$ and $f(\xi)=0 \forall \xi \in B\}$, and that the capacity of $A, B$ is defined by

$$
\operatorname{cap}(A, B):=\inf \{D(f): f \in \mathcal{C}(A, B)\}
$$

As $\max \{D(f \wedge 1), D(f \vee 0)\} \leq D(f), \forall f \in L^{2}(M)$, we may restrict the infimum to functions bounded below by 0 and bounded above by 1 .

Denote by $f_{A B}: E \rightarrow \mathbb{R}$ the function in $\mathcal{C}(A, B)$ defined as

$$
f_{A B}(\eta):=\mathbf{P}_{\eta}\left[T_{A}<T_{B}\right] \text {. }
$$


An elementary computation shows that $f_{A B}$ solves the equation

$$
\begin{cases}(L f)(\eta)=0 & \eta \in E \backslash(A \cup B), \\ f(\eta)=1 & \eta \in A \\ f(\eta)=0 & \eta \in B .\end{cases}
$$

Clearly, we may replace the generator $L$ by the operator $I-P$ in the above equation. It is not difficult to show that (6.15) has a unique solution in $L^{2}(M)$ given by $f_{A B}$. Indeed, if $f, g$ are solutions, $D(f-g)=\langle(I-P)(f-g),(f-g)\rangle_{M}=0$. In particular, by the explicit expression of the Dirichlet form, $f-g$ is constant. Since the difference vanishes on $A \cup B, f=g$.

Lemma 6.6. For any two disjoint subsets $A, B$ of $E$,

$$
\operatorname{cap}(A, B)=D\left(f_{A B}\right)=\sum_{\eta \in A} M(\eta) \mathbf{P}_{\eta}\left[T_{B}^{+}<T_{A}^{+}\right] .
$$

Proof. We first claim that there exists a function $f$ in $\mathcal{C}(A, B)$ whose Dirichlet form is equal to the capacity $\operatorname{cap}(A, B)$. Indeed, we have already seen that we may restrict the variational problem defining the capacity to functions bounded below by 0 and bounded above by 1 . Consider a sequence $\left\{f_{n}: n \geq 1\right\}$ in $\mathcal{C}(A, B)$ such that $0 \leq f_{n} \leq 1, \lim _{n \rightarrow \infty} D\left(f_{n}\right)=\operatorname{cap}(A, B)$. Since the sequence $f_{n}$ is uniformly bounded, there exist $f$ in $\mathcal{C}(A, B), 0 \leq f \leq 1$, and a subsequence, still denoted by $\left\{f_{n}: n \geq 1\right\}$, such that $f(\eta)=\lim _{n \rightarrow \infty} f_{n}(\eta)$ for every $\eta$ in $E$. By Fatou's lemma, $D(f) \leq \liminf _{n \rightarrow \infty} D\left(f_{n}\right)=\operatorname{cap}(A, B)$. Since $f$ belongs to $\mathcal{C}(A, B)$, $D(f)=\operatorname{cap}(A, B)$, which proves the claim.

We further claim that $f$ solves (6.15). Fix $\eta \notin A \cup B$. Since $f$ solves the variational problem for the capacity, it is clear that $f(\eta)$ is the argument which minimizes the convex function $F: \mathbb{R} \rightarrow \mathbb{R}$ defined by

$$
F(a)=\sum_{\xi \sim \eta} M(\eta) p(\eta, \xi)\{f(\xi)-a\}^{2} .
$$

In this formula $\xi \sim \eta$ means that the underlying jump chain may jump from $\eta$ to $\xi$, i.e., that $p(\eta, \xi)>0$. An elementary computation shows that the minimum is attained at $a=\sum_{\xi} p(\eta, \xi) f(\xi)$ so that $f(\eta)=(P f)(\eta)$. Since $f_{A B}$ is the unique solution in $L^{2}(M)$ of (6.15), $f=f_{A B}$ and $\operatorname{cap}(A, B)=D\left(f_{A B}\right)$. This proves the first statement of the lemma. The second one follows from a straightforward computation.

In particular, by (6.13) we have the following very useful identity between capacities and mean set rates.

Lemma 6.7. Assume that $F=A \cup B$ and $A \cap B=\varnothing$. Then,

$$
\mu(A) r_{F}(A, B)=\operatorname{cap}(A, B) .
$$

Next result shows that the mean set rates can be expressed in terms of capacities.

Lemma 6.8. Let $A, B$ be subsets of $F$ such that $A \cap B=\varnothing$. Then,

$$
\mu(A) r_{F}(A, B)=\frac{1}{2}\{\operatorname{cap}(A, F \backslash A)+\operatorname{cap}(B, F \backslash B)-\operatorname{cap}(A \cup B, F \backslash[A \cup B])\} .
$$

Proof. The proof is elementary and follows from Lemma 6.7 and the identity

$$
\begin{aligned}
2 \mu(A) r_{F}(A, B) & =\mu(A) r_{F}(A, F \backslash A)+\mu(B) r_{F}(B, F \backslash B) \\
& -\mu(A \cup B) r_{F}(A \cup B, F \backslash[A \cup B]) .
\end{aligned}
$$


By assumption (6.14), the holding rates $\lambda: E \rightarrow \mathbb{R}_{+}$belong to $L^{1}(\mu)$. This property extends to the holding rates $\left\{\lambda^{h}(\eta): \eta \in E\right\}$ of the $h$-trace process if $h$ belongs to $L^{1}(\mu)$. Indeed, by (6.12) and (6.9),

$$
\sum_{\eta \in E} \lambda^{h}(\eta) \mu^{h}(\eta)=\frac{1}{\langle h\rangle_{\mu}} \sum_{\eta \in E} M(\eta) \mathbf{P}_{\eta}\left[T_{F}^{+}=T_{F \backslash\{\eta\}}^{+}\right]<\infty .
$$

Therefore, assumption (6.14) holds for the $h$-trace process whenever $h$ belongs to $L^{1}(\mu)$. In this case, its capacity, denoted by $\operatorname{cap}_{h}(\cdot, \cdot)$, is well defined. Next result shows a simple relation between $\operatorname{cap}_{h}(\cdot, \cdot)$ and the capacity of the original process.

Lemma 6.9. Let $h: E \rightarrow \mathbb{R}_{+}$be a nonnegative $\mu$-integrable function with nonempty support denoted by $F$. Then, for every subsets $A, B$ of $F, A \cap B=\varnothing$,

$$
\langle h\rangle_{\mu} \operatorname{cap}_{h}(A, B)=\operatorname{cap}(A, B) .
$$

Proof. Fix a function $h: E \rightarrow \mathbb{R}_{+}$with the properties required in the statement of the lemma and two subsets $A, B$ of $F$ such that $A \cap B=\varnothing$. By Lemma 6.6 applied to the process $\left\{\eta_{t}^{h}: t \geq 0\right\}$ and by identities (6.8), (6.12) and (6.9),

$$
\begin{aligned}
\operatorname{cap}_{h}(A, B) & =\sum_{\eta \in A} \mu^{h}(\eta) \lambda^{h}(\eta) \mathbf{P}_{\eta}\left[\tau_{B}^{+}\left(\eta^{h}\right)<\tau_{A}^{+}\left(\eta^{h}\right)\right] \\
& =\frac{1}{\langle h\rangle_{\mu}} \sum_{\eta \in A} M(\eta) \mathbf{P}_{\eta}\left[T_{B}^{+}<T_{A}^{+} \mid T_{F}^{+}=T_{F \backslash\{\eta\}}\right] \mathbf{P}_{\eta}\left[T_{F}^{+}=T_{F \backslash\{\eta\}}^{+}\right] .
\end{aligned}
$$

Since for $\eta \in A$, the event $\left\{T_{B}^{+}<T_{A}^{+}\right\}$is contained in the event $\left\{T_{F}^{+}=T_{F \backslash\{\eta\}}\right\}$ $\mathbf{P}_{\eta}$-almost surely, the previous expression is equal to

$$
\frac{1}{\langle h\rangle_{\mu}} \sum_{\eta \in A} M(\eta) \mathbf{P}_{\eta}\left[T_{B}^{+}<T_{A}^{+}\right]
$$

By Lemma 6.6 this expression is equal to $\langle h\rangle_{\mu}^{-1} \operatorname{cap}(A, B)$, which proves the lemma.

We conclude this subsection proving a relation between expectations of time integrals of functions and capacities. Fix two disjoint subsets $A, B$ of $E$. Define the probability measure $\nu_{A B}$ on $A$ as

$$
\nu_{A B}(\eta)=\frac{M(\eta) \mathbf{P}_{\eta}\left[T_{B}^{+}<T_{A}^{+}\right]}{\operatorname{cap}(A, B)}, \quad \eta \in A .
$$

Denote by $\mathbf{E}_{\nu_{A B}}$ the expectation associated to the Markov process $\left\{\eta_{t}: t \geq 0\right\}$ with initial distribution $\nu_{A B}$. The proof of the following proposition is an adaptation of the proof of identity (4.28) in [12.

Proposition 6.10. Fix two disjoint subsets $A, B$ of $E$. Let $g: E \rightarrow \mathbb{R}$ be a $\mu$-integrable function. Then,

$$
\mathbf{E}_{\nu_{A B}}\left[\int_{0}^{T_{B}} g\left(\eta_{t}\right) d t\right]=\frac{\left\langle g, f_{A B}\right\rangle_{\mu}}{\operatorname{cap}(A, B)} .
$$


Proof. We first claim that the proposition holds for indicator functions of states. Fix an arbitrary state $\xi \in E$. Consider the random time $t_{B}:=\inf \left\{n \geq 0: Y_{n} \in B\right\}$ and the last exit time

$$
L_{A B}:=\sup \left\{n \geq 0: Y_{n} \in A \text { and } n<t_{B}\right\}
$$

with the convention that $\sup \varnothing=-\infty$. Then,

$$
\begin{aligned}
\mathbf{P}_{\xi}\left[T_{A}<T_{B}\right] & =\sum_{n \geq 0} \mathbf{P}_{\xi}\left[L_{A B}=n\right] \\
& =\sum_{n \geq 0} \sum_{\eta \in A} \mathbf{P}_{\xi}\left[Y_{n}=\eta ; n<t_{B}\right] \mathbf{P}_{\eta}\left[T_{B}^{+}<T_{A}^{+}\right] \\
& =\sum_{\eta \in A} \mathbf{P}_{\eta}\left[T_{B}^{+}<T_{A}^{+}\right] \sum_{n \geq 0} \mathbf{P}_{\xi}\left[Y_{n}=\eta ; n<t_{B}\right] .
\end{aligned}
$$

Since $Y$ is reversible with respect to $M$, the last expression is equal to

$$
\sum_{\eta \in A} \mathbf{P}_{\eta}\left[T_{B}^{+}<T_{A}^{+}\right] \frac{M(\eta)}{M(\xi)} \sum_{n \geq 0} \mathbf{P}_{\eta}\left[Y_{n}=\xi ; n<t_{B}\right] .
$$

Recall from the beginning of this section that $\left\{e_{n}: n \geq 0\right\}$ is a sequence of i.i.d. mean one exponential random variables independent of the jump chain $\left\{Y_{n}: n \geq 0\right\}$. By definition of the measure $\nu_{A B}$, this sum can be rewritten as

$$
\begin{aligned}
& \operatorname{cap}(A, B) \sum_{\eta \in A} \nu_{A B}(\eta) \frac{\lambda(\xi)}{M(\xi)} \mathbf{E}_{\eta}\left[\sum_{n=0}^{t_{B}-1} \frac{e_{n}}{\lambda(\xi)} \mathbf{1}\left\{Y_{n}=\xi\right\}\right] \\
& =\frac{\operatorname{cap}(A, B)}{\mu(\xi)} \mathbf{E}_{\nu_{A B}}\left[\int_{0}^{T_{B}} \mathbf{1}\left\{\eta_{s}=\xi\right\} d s\right] .
\end{aligned}
$$

This proves the assertion for $g=\mathbf{1}\{\xi\}$. By linearity and the monotone convergence theorem we get the desired result for positive and then $\mu$-integrable functions.

In particular, taking $A=\{\eta\}$ and $B=\{\xi\}$ for $\eta \neq \xi$ we have that

$$
\mathbf{E}_{\eta}\left[\int_{0}^{T_{\xi}} g\left(\eta_{s}\right) d s\right]=\frac{\left\langle g, f_{\{\eta\}\{\xi\}}\right\rangle_{\mu}}{\operatorname{cap}(\{\eta\},\{\xi\})}
$$

for any $\mu$-integrable function $g$.

This formula provides a more accurate estimate in Corollary 6.5 in the reversible context. For each $x \in S$, let

$$
\operatorname{cap}\left(\xi_{x}\right):=\inf _{\eta \in A^{x} \backslash\left\{\xi_{x}\right\}} \operatorname{cap}\left(\{\eta\},\left\{\xi_{x}\right\}\right) .
$$

Lemma 6.11. Let $g: E \rightarrow \mathbb{R}$ be a function integrable with respect to $\mu$. If the measure $\mu$ is reversible then, for each $x \in S$,

$$
\sup _{\eta \in A^{x}} \mathbf{E}_{\eta}\left[\int_{0}^{T_{\xi_{x}}}\left|g^{x}\left(\eta_{s}\right)\right| d s\right] \leq \frac{2\langle|g|\rangle_{\mu^{x}}}{\operatorname{cap}\left(\xi_{x}\right)} \mu\left(A^{x}\right),
$$

where $|g|(\eta)=|g(\eta)|$ for all $\eta$ in $E$.

Proof. By (6.16) and the fact that $0 \leq f_{\{\eta\}\left\{\xi_{x}\right\}} \leq 1$, the left hand side is bounded above by

$$
\sup _{\eta \in A^{x} \backslash\left\{\xi^{x}\right\}} \frac{\left\langle\left|g^{x}\right|, f_{\{\eta\}\left\{\xi_{x}\right\}}\right\rangle_{\mu}}{\operatorname{cap}\left(\{\eta\},\left\{\xi_{x}\right\}\right)} \leq \frac{\left\langle\left|g^{x}\right|\right\rangle_{\mu}}{\operatorname{cap}\left(\xi_{x}\right)} \leq \frac{2\left\langle|g| \mathbf{1}\left\{A^{x}\right\}\right\rangle_{\mu}}{\operatorname{cap}\left(\xi_{x}\right)}
$$


for each $x \in S$. This completes the proof.

Acknowledgments: The authors would like to thank E. Olivieri for fruitful discussions on metastability and the two anonymous referees for their suggestions.

\section{REFERENCES}

[1] I. Armendariz, M. Loulakis. Thermodynamic limit for the invariant measures in supercritical zero range processes. preprint (2008).

[2] P. Billingsley, Convergence of Probability Measures, 2nd Edition. John Wiley \& Sons, 1999.

[3] J. Beltrán, C. Landim: Metastability of reversible condensed zero-range processes on complete graphs. preprint (2009).

[4] J. Beltrán, C. Landim: Metastability of reversible condensed zero-range processes on finite graphs. preprint (2009).

[5] A. Bovier. Metastability, Lectures given at the 5th Prague Summer School on Mathematical Statistical Physics, 2006. Online available at http://www.wias-berlin.de/people/bovier/files/prague.pdf

[6] M. Cassandro, A. Galves, E. Olivieri, M. E. Vares. Metastable behavior of stochastic dynamics: A pathwise approach. J. Stat. Phys. 35, 603-634 (1984).

[7] K. L. Chung, Markov chains with stationary transition probabilities. Second edition. Die Grundlehren der mathematischen Wissenschaften, Band 104 Springer-Verlag New York, Inc., New York 1967.

[8] M. R. Evans. Phase transitions in one-dimensional nonequilibrium systems. Braz. J. Phys. 30, 42-47 (2000).

[9] M. R. Evans, S. N. Majumdar, R. K. P. Zia: Canonical analysis of condensation in factorized steady states. J. Stat. Phys. 123, 357-390 (2006)

[10] P. A. Ferrari, C. Landim, V. V. Sisko. Condensation for a fixed number of independent random variables. J. Stat. Phys. 128, 1153-1158 (2007).

[11] David Freedman. Markov chains. Holden-Day, San Francisco (1971).

[12] A. Gaudillière. Condenser physics applied to Markov chains: A brief introduction to potential theory. Online available at http://arxiv.org/abs/0901.3053

[13] S. Großkinsky, G. M. Schütz, H. Spohn. Condensation in the zero range process: stationary and dynamical properties. J. Statist. Phys. 113, 389-410 (2003)

[14] I. Jeon, P. March, B. Pittel: Size of the largest cluster under zero-range invariant measures. Ann. Probab. 28, 1162-1194 (2000)

[15] C. Kipnis, C. Landim. Scaling limits of interacting particle systems, Grundlehren der Mathematischen Wissenschaften [Fundamental Principles of Mathematical Sciences], vol. 320. Springer-Verlag, Berlin (1999)

[16] J. L. Lebowitz, O. Penrose: Rigorous treatment of the van der Waals-Maxwell theory of the liquid-vapor transition. J. Math. Phys. 7, 98-113 (1966).

[17] J. R. Norris. Markov chains. Cambridge University Press, Cambridge (1997).

[18] E. Olivieri and M. E. Vares. Large deviations and metastability. Encyclopedia of Mathematics and its Applications, vol. 100. Cambridge University Press, Cambridge, 2005.

[19] E. Scoppola. Renormalization group for Markov chains and application to metastability. J. Stat. Phys. 73, 83-121 (1993).

imCa, Calle los Biólogos 245, Urb. San César Primera Etapa, lima 12, Perú and PUCP, Av. Universitaria cdra. 18, San Miguel, Ap. 1761, Lima 100, Perú.

E-MAIL: johel@impa.br

impa, Estrada Dona Castorina 110, CEP 22460 Rio de Janeiro, Brasil and CNRS UMR 6085, Université de Rouen, Avenue de l'Université, BP.12, Technopôle du MadrilLET, F76801 SAInt-Étienne-DU-Rouvray, France.

E-MAIL: landim@impa.br 